Florida International University FIU Digital Commons

$11-22-1993$

\title{
Three dimensional stress intensity factor for large arrays of radial internal surface cracks in a cylindrical pressure vessel
}

Javier Pierola

Florida International University College of Law

DOI: $10.25148 /$ etd.FI15103199

Follow this and additional works at: https://digitalcommons.fiu.edu/etd

Part of the Mechanical Engineering Commons

\section{Recommended Citation}

Pierola, Javier, "Three dimensional stress intensity factor for large arrays of radial internal surface cracks in a cylindrical pressure vessel" (1993). FIU Electronic Theses and Dissertations. 2514.

https://digitalcommons.fiu.edu/etd/2514 
FLORIDA INTERNATIONAL UNIVERSITY

Miami, Florida

\section{THREE DIMENSIONAL STRESS INTENSITY FACTOR FOR \\ LARGE ARRAYS OF RADIAL INTERNAL SURFACE CRACKS \\ IN A CYLINDRICAL PRESSURE VESSEL}

A thesis submitted in partial satisfaction of the

requirements for the degree of

MASTER OF SCIENCE

IN

MECHANICAL ENGINEERING

by

Javier Pierola 
To : Gordon R. Hopkins

College of Engineering

This thesis, written by Javier Pierola, and entitled Three dimensional Stress Intensity Factors for Large Arrays of Internal Surface Cracks in a Cylindrical Pressure Vessel, having been approved in respect to style and intellectual content, is referred to you for judgement.

We have read this thesis and recommend that it be approved

M. Ebadian

M.Perl

K. Wu

M. EI-Sayed

C. Levy, Major Professor

Date of Defense: November 22, 1993

The thesis of Javier Pierola is approved

Dean Gordon R. Hopkins

Division of Graduate Studies

Dr. Richard Campbell

Dean of Graduate Students

Florida International University, 1993 
A very special dedication to my wife Carmen, without her encouragement, understanding, and love, the completion of this work would not have been possible. I, also, want to dedicate this thesis to my parents for their great support. 


\section{ACKNOWLEDGMENTS}

I wish to thank the members of my committee for their helpful comments and patience. Special thanks to my advisors Dr.Levy and Dr.Perl for their dedication to the correction of this thesis and for their invaluable support and guidance throughout the master's program. 
ABSTRACT OF THE THESIS

THREE DIMENSIONAL STRESS INTENSITY FACTORS FOR

LARGE ARRAYS OF RADIAL INTERNAL SURFACE CRACKS IN

A CYLINDRICAL PRESSURE VESSEL

by

\author{
Javier Pierola \\ Florida International University \\ Miami,Florida \\ Cesar Levy, Major Professor
}

The objective of this study is to present the mode I stress intensity factor distribution (SIF) along the crack-front for a wide array of semicircular and semi-elliptical surface cracks inside of a pressurized thickwalled cylinder. A three-dimensional finite element package ANSYS is used to evaluate the SIF for multicracked cylinder with number of cracks from $n=1$ to 128 , the ratio of crack-depth to the wall thickness $a / t=0.05$ to 0.6 , the ellipticity of the crack (the crack-depth to the semi-crack length) $a / c=0.2$ to 1.5 , the ratio of the outer to the inner radius $r_{o} / r_{i}=2$.

A substructuring technique is introduced which solved a coarse model meshed with ten-node isoparametric elements and applied the 
resulting displacements in the boundary surface of a submodel which is built employing singular elements along the crack-front to produce the $1 / \sqrt{\mathrm{r}}$ singularity. The SIF is evaluated using nodal-displacement method.

To validate the modeling and analysis procedure of the present results various configurations were solved using this method and compared to other finite element solutions. The present results were in very good agreement: less than $5 \%$ comparing with Raju and Newman's results and within $8 \%$ of Kirkhope's results.

An empirical equation to calculate the maximum SIF, was developed in this study. The equation was obtained by nonlinear fitting of the finite element results and the error was within $\pm 5.7 \%$. 


\section{TABLE OF CONTENTS}

CHAPTER

PAGE

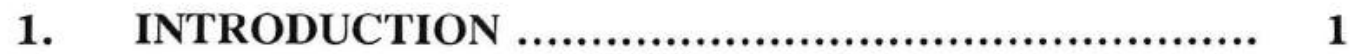

1.1 General ................................................ 1

1.2 Literature Survey ................................... 2

1.3 Statement of the problem ........................... 4

2. FRACTURE MECHANICS ............................ 6

2.1 General .............................................. 6

2.2 A crack in a structure ............................... 7

2.3 Stress intensity factor (SIF or $K_{l}$ ) $\ldots \ldots \ldots \ldots \ldots \ldots \ldots . .6$

2.4 Plane stress and Plane strain ......................... 12

3. NUMERICAL METHOD ............................. 15

3.1 Finite Element Method (FEM) ..................... 15

3.2 Singular Elements ................................. 18

3.3 Numerical Process ................................... 22

3.4 Maximum stress intensity factor equation ........... 34

4. $\quad$ RESULTS ............................................ 37

4.1 Validation of the model.............................. 37

4.2 Results ............................................ 41

4.3 Empirical equation results .......................... 43

5. ANALYSIS AND DISCUSSION ....................... 44

5.1 Semi-circular surface cracks ....................... 44

5.2 Semi-elliptical surface cracks ....................... 48

5.3 Conclusions ........................................ 61

5.4 Future research ................................... 63

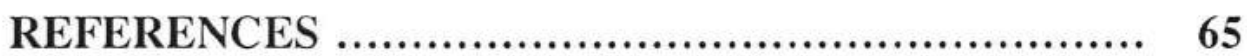

APPENDIX A: Tables of normalized SIF .............. 70

APPENDIX B: Normalized SIF vs. $\phi \ldots \ldots \ldots \ldots \ldots \ldots \ldots . . \ldots 2$

APPENDIX C: Macros ............................... 94

APPENDIX D: FEM SIF vs. empirical equation .......... 99 


\section{LIST OF FIGURES}

Figure 2.1 The three modes of loading 9

Figure 2.2 Plastic zone at the crack tip 11

Figure 2.3 Thick plate under mode I loading 13

Figure 3.1 Two-dimensional eight-node isoparametric elements 19

Figure 3.2 Semi-elliptical surface crack in a cylinder 24

Figure 3.3 The model meshed with ten-node tetrahedral elements 25

Figure 3.4 The submodel meshed with twenty-node solid elements 27

Figure 3.5 Measure of the angle $\phi$ for semi-elliptical cracks 28

Figure 3.6 Nodes used for the crack front displacements 33

Figure 4.1 Comparison of stress intensity factor along crack front for two semi-circular surface cracks in pressurized cylindrical vessel

Figure 4.2 Comparison of stress intensity factor along crack front for two semi-elliptical surface cracks in pressurized cylindrical vessel 39

Figure 4.3 Number of elements necessary to achieve convergence 40

Figure 5.1 Stress intensity factor along crack front for semi-circular surface cracks in pressurized cylindrical vessel $(a / t=0.05)$

Figure 5.2 Stress intensity factor along crack front for semi-circular surface cracks in pressurized cylindrical vessel $(a / t=0.2)$

Figure 5.3 Stress intensity factor along crack front for semi-circular surface cracks in pressurized cylindrical vessel $(a / t=0.6)$

Figure 5.4 Stress intensity factor along crack front for semi-circular surface cracks in pressurized cylindrical vessel ( $n=2$ cracks)

Figure 5.5 Maximum stress intensity factor along crack front for semi-circular surface cracks in pressurized cylindrical vessel

Figure 5.6 Stress intensity factor along crack front for semi-elliptical surface cracks in pressurized cylindrical vessel $(a / c=0.5, a / t=0.05)$ 
Figure 5.7 Stress intensity factor along crack front for semi-elliptical surface cracks in pressurized cylindrical vessel $(a / c=0.5, a / t=0.2)$

Figure 5.8 Stress intensity factor along crack front for semi-elliptical surface cracks in pressurized cylindrical vessel $(a / c=0.5, a / t=0.6)$

Figure 5.9 Stress intensity factor along crack front for semi-elliptical surface cracks in pressurized cylindrical vessel $(a / c=0.5)$

Figure 5.10 Maximum stress intensity factor along crack front for semi-elliptical surface cracks in pressurized cylindrical vessel $(a / c=0.5)$

Figure 5.11 Stress intensity factor along crack front for semi-elliptical surface cracks in pressurized cylindrical vessel $(a / c=0.2, a / t=0.05)$

Figure 5.12 Stress intensity factor along crack front for semi-elliptical surface cracks in pressurized cylindrical vessel $(a / c=0.2, a / t=0.1)$

Figure 5.13 Stress intensity factor along crack front for semi-elliptical surface cracks in pressurized cylindrical vessel $(a / c=0.2, a / t=0.2)$

Figure 5.14 Stress intensity factor along crack front for semi-elliptical surface cracks in pressurized cylindrical vessel $(a / c=1.5, a / t=0.05)$

Figure 5.15 Stress intensity factor along crack front for semi-elliptical surface cracks in pressurized cylindrical vessel $(a / c=1.5, a / t=0.2)$

Figure 5.16 Stress intensity factor along crack front for semi-elliptical surface cracks in pressurized cylindrical vessel $(a / c=1.5, a / t=0.6)$

Figure 5.17 Stress intensity factor along crack front for semi-elliptical surface cracks in pressurized cylindrical vessel $(a / c=1.5)$

Figure 5.18 Stress intensity factor along crack front for two surface cracks in pressurized $(a / t=0.05)$ of different ellipticity as a function of the crack depth

Figure 5.19 Stress intensity factor along crack front for two surface cracks in pressurized $(a / t=0.2)$ of different ellipticity as a function of the crack 
depth

Figure 5.20 Variation of the stress intensity factor as a function of $t / r_{i}$

60

Figure B.1 Normalized stress intensity factor vs. $\phi$

$(a / c=0.2, a / t=0.05)$

83

Figure B.2 Normalized stress intensity factor vs. $\phi$ $(a / c=0.2, a / t=0.1)$

83

Figure B.3 Normalized stress intensity factor vs. $\phi$ $(a / c=0.2, a / t=0.15)$

84

Figure B.4 Normalized stress intensity factor vs. $\phi$ $(a / c=0.2, a / t=0.2)$

Figure B.5 Normalized stress intensity factor vs. $\phi$ $(a / c=0.5, a / t=0.05)$

Figure B.6 Normalized stress intensity factor vs. $\phi$ $(a / c=0.5, a / t=0.1)$

Figure B.7 Normalized stress intensity factor vs. $\phi$ $(a / c=0.5, a / t=0.15)$

Figure B.8 Normalized stress intensity factor vs. $\phi$ $(a / c=0.5, a / t=0.2)$

Figure B.9 Normalized stress intensity factor vs. $\phi$ $(a / c=0.5, a / t=0.4)$

Figure B.10 Normalized stress intensity factor vs. $\phi$ $(a / c=0.5, a / t=0.6)$

Figure B.11 Normalized stress intensity factor vs. $\phi$ $(a / c=1, a / t=0.05)$

Figure B.12 Normalized stress intensity factor vs. $\phi$ $(a / c=1, a / t=0.1)$

Figure B.13 Normalized stress intensity factor vs. $\phi$ $(a / c=1, a / t=0.15)$

Figure B.14 Normalized stress intensity factor vs. $\phi$ $(a / c=1, a / t=0.2)$

Figure B.15 Normalized stress intensity factor vs. $\phi$ $(a / c=1, a / t=0.4)$

Figure B.16 Normalized stress intensity factor vs. $\phi$ $(a / c=1, a / t=0.6)$

Figure B.17 Normalized stress intensity factor vs. $\phi$ $(a / c=1.5, a / t=0.05)$

Figure B.18 Normalized stress intensity factor vs. $\phi$ $(a / c=1.5, a / t=0.1)$

Figure B.19 Normalized stress intensity factor vs. $\phi$ 


$$
(a / c=1.5, a / t=0.15) \quad 92
$$

Figure B.20 Normalized stress intensity factor vs. $\phi$ $(a / c=1.5, a / t=0.2)$

Figure B.21 Normalized stress intensity factor vs. $\phi$

$$
(a / c=1.5, a / t=0.4)
$$

93

Figure B.22 Normalized stress intensity factor vs. $\phi$ $(a / c=1.5, a / t=0.6)$ 


\section{LIST OF TABLES}

Table 4.1 Crack configurations solved in the present study 42

Table 4.2 Values of the coefficients $b_{i}$ (equation 3.26)

43

Table A.1 Normalized SIF for various number of cracks $(a / c=0.2, a / t=0.05)$

Table A.2 Normalized SIF for various number of cracks $(a / c=0.2, a / t=0.1)$

Table A.3 Normalized SIF for various number of cracks $(a / c=0.2, a / t=0.15)$

Table A.4 Normalized SIF for various number of cracks $(a / c=0.2, a / t=0.2)$

Table A.5 Normalized SIF for various number of cracks $(a / c=0.5, a / t=0.05)$

Table A.6 Normalized SIF for various number of cracks $(a / c=0.5, a / t=0.1)$

Table A.7 Normalized SIF for various number of cracks $(a / c=0.5, a / t=0.15)$

Table A.8 Normalized SIF for various number of cracks $(a / c=0.5, a / t=0.2)$

Table A.9 Normalized SIF for various number of cracks $(a / c=0.5, a / t=0.4)$

Table A.10 Normalized SIF for various number of cracks $(a / c=0.5, a / t=0.6)$

Table A.11 Normalized SIF for various number of cracks $(a / c=1, a / t=0.05)$

Table A.12 Normalized SIF for various number of cracks $(a / c=1, a / t=0.1)$

Table A.13 Normalized SIF for various number of cracks $(a / c=1, a / t=0.15)$

Table A.14 Normalized SIF for various number of cracks $(a / c=1, a / t=0.2)$

Table A.15 Normalized SIF for various number of cracks $(a / c=1, a / t=0.4)$

Table A.16 Normalized SIF for various number of cracks $(a / c=1, a / t=0.6)$

Table A.17 Normalized SIF for various number of cracks $(a / c=1.5, a / t=0.05)$

Table A.18 Normalized SIF for various number of cracks 


$$
(a / c=1.5, a / t=0.1)
$$

Table A.19 Normalized SIF for various number of cracks $(a / c=1.5, a / t=0.15)$

Table A.20 Normalized SIF for various number of cracks $(a / c=1.5, a / t=0.2)$

Table A.21 Normalized SIF for various number of cracks $(a / c=1.5, a / t=0.4)$

Table A.22 Normalized SIF for various number of cracks $(a / c=1.5, a / t=0.6)$ 


\section{LIST OF SYMBOLS}

$\begin{array}{ll}a & \text { Crack depth } \\ a & \text { Field variable vector } \\ C_{o} & \text { Normalization factor } \\ c & \text { Semi-crack length } \\ f & \text { Force vector } \\ G & \text { Shear modulus } \\ i, j, k \ldots & \text { Node number } \\ \boldsymbol{K} & \text { Stiffness matrix } \\ K_{I} & \text { Mode I stress intensity factor } \\ K_{I I} & \text { Mode II stress intensity factor } \\ K_{I I I} & \text { Mode III stress intensity factor } \\ L & \text { Length of the cylinder } \\ N_{I} & \text { Shape functions } \\ n & \text { Number of cracks } \\ r_{i} & \text { Inner radius of the cylinder } \\ Q & \text { Shape of an ellipse } \\ r_{o} & \text { Outer radius of the cylinder } \\ s, t & \text { Serendipity coordinates } \\ t & \text { Thickness of the cylinder } \\ u & \text { Displacements in X-direction } \\ v & \text { Displacement in Y-direction } \\ w & \text { Displacement in Z-direction } \\ \epsilon_{x x} & \text { Strain in } x \text {-direction } \\ \epsilon_{y y} & \text { Strain in } y \text {-direction } \\ \epsilon_{z z} & \text { Strain in } z \text {-direction } \\ \sigma_{y} & \text { Yield stress } \\ \sigma_{x x} & \text { Stress in } x \text {-direction } \\ \sigma_{y y} & \text { Stress in } y \text {-direction } \\ \sigma_{z z} & \text { Stress in } z \text {-direction } \\ v & \text { Poisson's ratio } \\ \phi & \text { Angle along the crack front } \\ \Theta & \text { Angle of the model related to } n \text { by } \Theta=\pi / n \\ \kappa & \text { Conversion factor for plane strain and plane stress } \\ & \end{array}$




\section{Chapter 1. Introduction}

\subsection{General}

Reactor pressure vessels, gun barrels, chemical pressure vessels are among many important applications that can be found for thick-walled cylindrical pressure vessels. Due to several factors, their particular geometrical configuration, the types of loading they are subjected to, and the environmental conditions, these thick-walled pressure vessels are very susceptible to internal cracking.

Most gun barrels presently in service life are rifled. As a consequence of this manufacturing process, stress concentration zones appear at the corners between the rifling and the land. During the firing process, the internal surface is exposed to the combined action of pressure pulses, extremes of temperature and an aggressive environment resulting from the combustion of the gun powder. This repetitive process can produce large arrays of radial cracks in the areas of stress concentration. 
In applications such as nuclear power plants (reactor pressure vessels) and chemical containment tanks (e.g. pressure vessels containing liquid fuel at very low temperature ) the pressure load, the corrosive agents and the service temperature are the principal factors that can contribute to the development of large arrays of radial cracks. A complete understanding of the crack behavior in such applications is very important because the failure can often be catastrophic.

\subsection{Literature survey}

Since the exact solution of radial cracks in a pressurized cylinder is very complex, previous investigators have used engineering estimates or numerical procedures to calculate the stress intensity factor (SIF) of such a cylinder.

Underwood [1] and Kobayashi [2] estimated the SIF for surface cracks in pressurized cylinders but without including the wall-thickness effect. However, Kobayashi et al. [3] estimated the SIF for inner and outer surface cracks in internally pressurized cylinders from the results of a semi-elliptical crack in a finite-thickness wall plate which did include the wall-thickness 
effect.

Most of the available numerical solutions were obtained using a two dimensional (2-D) model. Tracy [4] using the complex variable approach, solved the problem numerically for one, two and four evenly spaced cracks emanating from the inner surface of a circular ring. Baratta [5], employing a load relief factor, evaluated the SIF for arrays of up to 48 cracks. Pu and Houssain [6], using cubic isoparametric elements, calculated the SIF for arrays of 2 to 40 cracks and for different crack lengths and geometrical configurations of a cylinder. Perl and Aroné [7,8], using the finite element method (FEM), obtained the SIF for large arrays of radial cracks of up to 1024 cracks and for a wide range of nondimensional crack lengths of 0.01 to 0.625 . Perl and colleagues $[8,9]$, also employed the same method to study the problem of autofrettaged gun barrels and the problem of internal thermal shock arising in the firing process. These 2-D solutions model the crack-front as an straight line; in reality, cracks have curved fronts. For this reason the 2-D solutions are considered to be an upper bound to the stress intensity factors prevailing in real situations.

At present, very few three dimensional (3-D) SIF solutions for semicircular and semi-elliptical cracks are available. Atluri and Kathiresan [10] 
and McGowan and Raymund [11] obtained the SIF for only a single surface crack using 3-D numerical analysis. Raju and Newman [12] using the 3-D finite element method, presented a solution for two internal and external surface cracks in a pressurized cylinder for various load conditions. The SIFs were obtained from a nodal force method which uses the nodal point stresses correlated with the crack tip equations to calculate the SIF. This method is considered to give upper bound solutions. Kirkhope et al. [13,14], using a similar finite element approach, evaluated the SIF for arrays of up to 32 radial, surface cracks in a thick-walled cylinder. O'Donoghue et al. $[15,16]$ presented a solution for two, coplanar, semi-elliptical, surface cracks in a cylindrical pressure vessel.

\subsection{Statement of the problem}

All the solutions described above obtained the SIFs along the crack front for a limited range of configuration parameters. References $[10,11$ and 12] considered only one and two cracks. In references $[13,14]$ the analysis was limited to a maximum of 32 cracks.

The purpose of this research is to present the mode I SIF for large 
arrays of internal semi-circular and semi-elliptical surface radial cracks in a thick-walled, pressurized cylinder. The SIF along the crack front for arrays of up to 180 cracks are obtained, for ratios of crack depth to wall thickness from 0.05 to 0.6 , for ellipticities of the crack (the crack depth to the semi-crack length) from 0.2 to 1.5 , and for a thick-walled cylinder with a ratio of the outer to the inner radius of 2 .

In most of the real situations, it is very difficult to find an exact solution of the SIF. In particular, for a radially multicracked cylinder a theoretical SIF is not available. Therefore, a numerical method is needed to estimate it. To accomplish this approach, a 3-D finite element package, ANSYS, is used. A substructuring procedure is introduced which employs two different types of isoparametric elements: 10-node tetrahedral and 20node solid. These 20-node elements are collapsed to produce singular elements along the crack front. The SIF is calculated based on the displacement method. 


\section{Chapter 2. Fracture Mechanics}

\subsection{General}

Many accidents in the past have been caused due to material deficiencies in the form of pre-existing flaws [17]. In most of these accidents the design of the structures were made using a conventional design criteria which did not consider the problem of fracture. These criteria are based on tensile strength, yield strength and buckling stress and are adequate for many engineering applications, but they are insufficient when there is a crack in the structure. In this case, fractures can occur at stress levels that are below the design limit.

"The structural study which considers crack-extension behavior as a function of applied loads is called fracture mechanics. Particularly, it is called linear elastic fracture mechanics when such study does not involve large plastically yielded regions surrounding cracks" [18] .

As a result of considerable research efforts during the last three 
decades, linear elastic fracture mechanics has become a very useful tool in design, solving many practical engineering problems in failure analysis, material selection, structural life-prediction, and acceptance test.

\subsection{A crack in a structure}

When a crack develops in a structure, the application of repeated loads, and, in many cases, the presence of an aggressive environment will originate the growth of the crack with time. The larger the crack the higher the stress concentration induced by it, thus the rate of crack propagation will increase with time. Due to the presence of the crack, the strength of the structure decreases and it will continue to decrease progressively as the crack size increases. The crack will continue to grow until the strength has become so low that the failure occurs under normal service loading.

Predictions such as the residual strength as a function of crack-size, the size of crack that can be tolerated at the expected service load (critical crack size), the time that takes for a crack to grow from a certain initial size to the critical size and the size of pre-existing flaw that can be permitted at 
the beginning of the service life are among the principal objects of engineering fracture mechanics [19].

\subsection{Stress intensity factor (SIF or $K_{l}$ )}

There are three different modes of separation at the crack-tip, as shown in Fig. 2.1. In mode I, also called the"opening mode", the tensile component of stress is applied normal to the face of the crack. The displacements are perpendicular to the plane of the crack. In mode II, the shear component of the stress is applied in $x$-direction ("sliding mode"). The displacements are in the plane of the crack and perpendicular to the leading edge of the crack. In mode III, the "tearing mode" is caused by the shear component of the stress applied normal to the leading edge of the crack. The displacements are in z-direction.

Any crack deformation can be represented by the superposition of these three modes. In a cylinder under pressure, an internal crack will be stressed in mode I, thus the discussions here are limited to mode I. 


\section{Mode I}

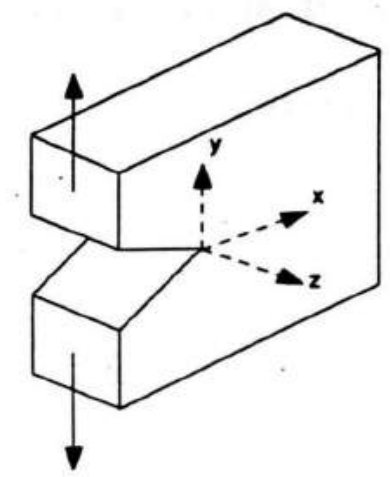

Mode II

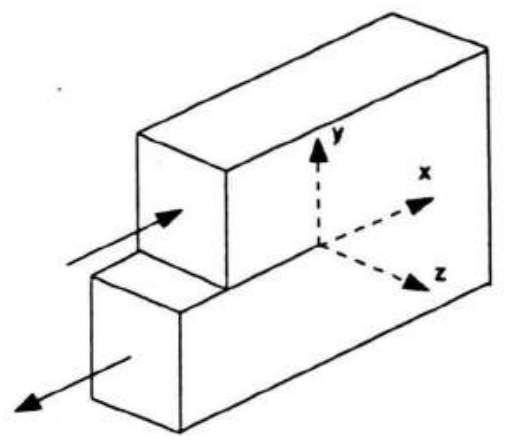

Mode III

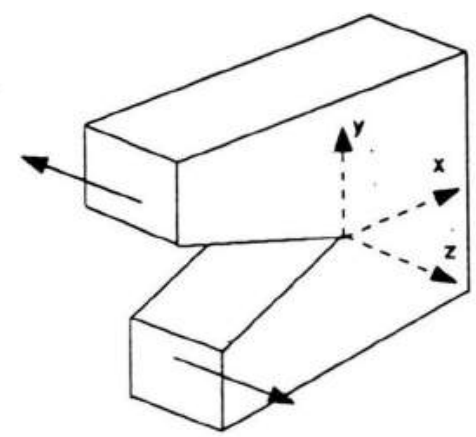

Fig.2.1 The three modes of loading 
For a crack of arbitrary size, $a$, in a body of arbitrary size and shape and loaded by arbitrary mode I loading, the stress field in the vicinity of the crack can be expressed as,

$$
\sigma_{i j}=\left(\frac{K_{I}}{\sqrt{2 \pi . r}}\right) f_{i j}(\theta)
$$

where, $\sigma_{i j}$ are the stresses acting on a material element $\mathrm{d} x \mathrm{~d} y$ at a distance $r$ from the crack tip, $K_{l}$ is the stress intensity factor in mode I, $\Theta$ an angle from the crack plane, and $f_{i j}(\Theta)$ are known functions of $\Theta$. Equation (2.1) represents the elastic solution for a two-dimensional crack. This equation also can be applied for our three-dimensional case along the crack front [26]. In reality, the stress does not become infinite at the crack-tip because as $\sigma$ reaches the yield stress $\sigma_{v}$, plastic deformation will occur creating a plastic zone in the vicinity of the crack tip as shown in Fig.2.2. 


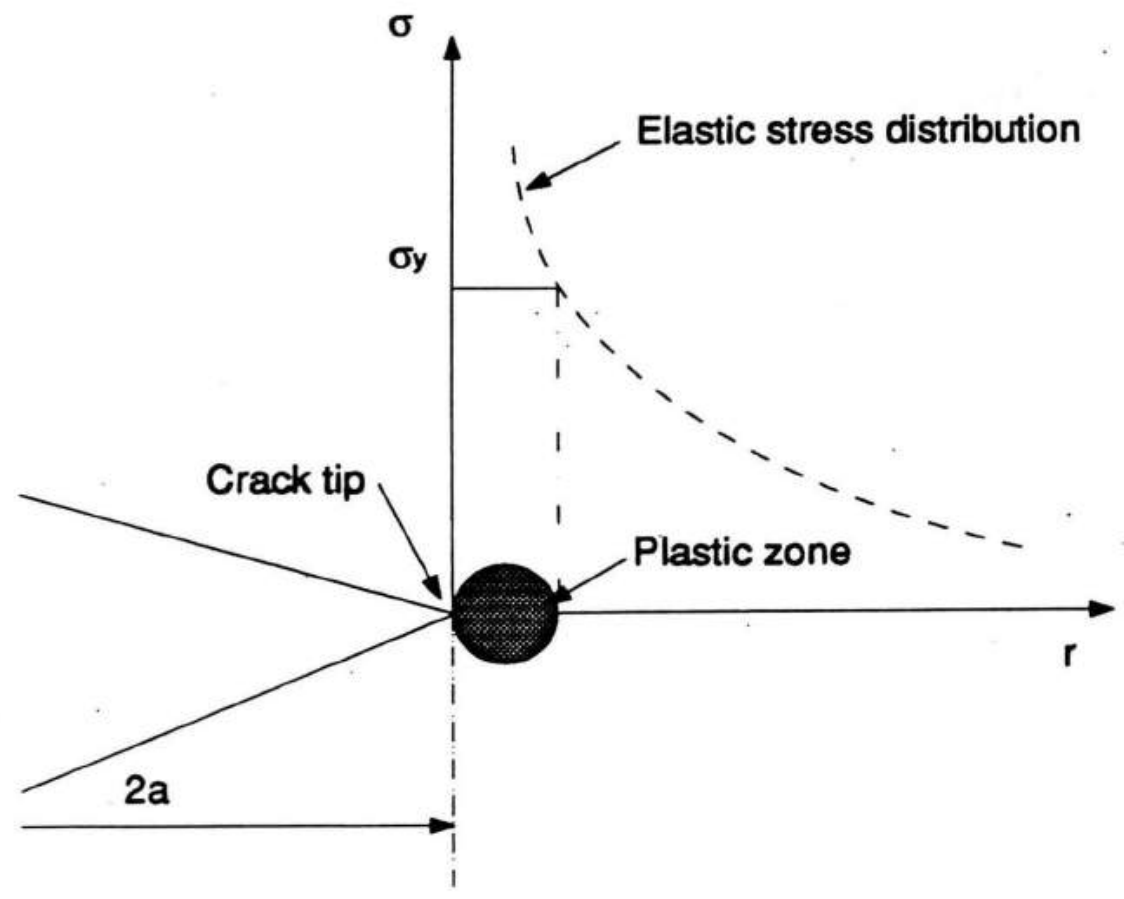

Fig. 2.2 Plastic zone at the crack tip.

This factor, $K_{l}$, is the most important parameter in fracture mechanics and is called the mode I stress intensity factor. $K$, describes the entire crack tip stress field. Similar solutions as (2.1) can be obtained for the other two modes.

The stress intensity factor $K_{l}$ is also a similitude parameter. This means that if there are two bodies of different size and shape, but of the same material, with cracks of different size, the two cracks will behave in the same manner if the stress intensity factor for both cases are equal. 
Knowledge of the crack-tip stress intensity factor as a function of applied load and geometry of the structure is necessary to predict the crackgrowth and the fatigue life of the structure. Once, the SIF is computed based on the principles of elasticity, the critical value of SIF is determined by experiment for the same material and the same mode of loading. If the theoretical SIF is less than the critical value by a safe margin then the crack is acceptable.

In most of the real situations, it is very difficult to find an exact solution of the SIF. In particular, for a radially, multicracked cylinder a theoretical SIF is not available. Therefore, a numerical method is needed to estimate it.

\subsection{Plane stress and Plane strain}

Consider a thick plate (Fig.2.3) with a crack subject to mode I loading. In the interior, the material around the crack tip will prevent the internal plastic zone from contracting; therefore, the interior will have 


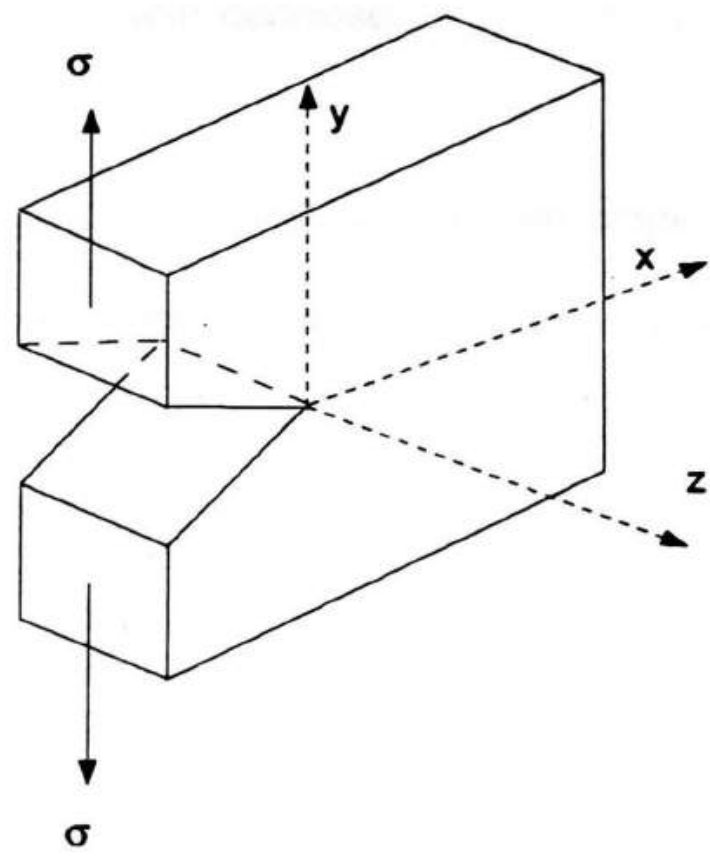

Fig.2.3 Thick plate under mode I loading

approximately a plane strain condition $\left(\epsilon_{: i}=0\right)$. It will be subjected to a stress in thickness direction expressed by:

$$
\sigma_{z z}=v\left(\sigma_{x x}+\sigma_{y y}\right)
$$


At the surface where there is no constraint, there will be no stress in the thickness direction $\left(\sigma_{z z}=0\right)$. With increasing thickness, the relative influence of the surface will decrease.

For the case of a three-dimensional semi-elliptical crack, plane strain condition will be considered through the interior. At the surface, a transition between plane strain and plane stress occurs. 


\section{Chapter 3. Numerical Method}

\subsection{Finite Element Method (FEM)}

The finite element method is one of the most powerful of the approximate solution methods, this method is applicable to problems with irregular geometries, heterogeneous and anisotropic materials, and different types of boundaries conditions. In fracture mechanics the finite element method offers a relatively simple process to determine the stress intensity factor for a variety of structures.

The basic idea of FEM is to divide or discretize the structure, body or region into a large number of finite elements. These elements may be one, two or three dimensional and are formed for different number of nodes. The FEM will provide the displacements (or temperature for heat transfer analysis) at each of these nodes using interpolation functions within each element to describe the variation of the displacement field as a function of the global coordinates. From the nodal displacements, the displacements at any point in the structure are easily determined and from these 
displacements, strain, stress and forces are also determined.

\subsubsection{General form for steady-state problems}

Since the problem analyzed in this study is a function of spacial coordinates only and not a function of time, the steady-state formulation of the FEM need only be reviewed. In all steady-state problems, a system of equations is always in the form:

$$
K a=f
$$

where $\boldsymbol{K}$ is the stiffness matrix, $\boldsymbol{a}$ is the field variable vector and $\boldsymbol{f}$ is the force vector. The field variable may be displacements in structural analysis, temperatures in thermal analysis and velocities or pressures in fluid flow problems. For structural analysis problems, the field variable is the displacement field. If sufficient boundary conditions are specified to guarantee a unique solution, equation (3.1) can be solved to obtain the displacements at each node in the structure (vector $a$ ). 


\subsubsection{Matrix Equation Solver}

There are different methods to solve the system of equations described above, the most common method are Gauss elimination and LU decomposition.

The ANSYS program uses the wave front or frontal solution procedure [20] to solve the system of equations generated by the finite element method. This wave front solver processes the elements sequentially, the program determines which element is the last to use each nodal point. As the total system of equations is assembled from the element matrices, the equations for a nodal point which occurs for the last time are solved in terms of the remaining unknowns and eliminated from the assembled stiffness matrix using Gauss elimination. As the solution progresses the equations for a node which occurs for first time are added to the assembled matrix.

The number of equations which are active after any element has been processed is called the wave front. Since this solution method analyzes the elements successively, the ordering of the elements is very important to 
minimize the wave front for reasons of efficiency and problem size. The computational time is proportional to the square of the wave front; besides, our version of ANSYS had a maximum wavefront of 800 allowed to solve any problem. ANSYS has a capability to reorder the elements to reduce the wave front and therefore to minimize the memory and time required to solve a problem.

\subsection{Singular Elements}

The most important step in the numerical analysis of linear elastic fracture mechanics is the selection of the element type around the crack tip ( for two-dimensional cracks) or crack front (for three dimensional cracks). As explained before, the stress in the crack tip (or crack-front) tends to infinity and the stress intensity factor $\left(K_{l}\right)$ is the parameter that describes the stress field near the crack. Therefore, it is necessary to have an element which describes the same inverse square root singularity of linear fracture mechanics. Although, a regular element can be used to obtain the stress intensity factors, the convergence rate is greatly retarded [21], even when utilizing higher order polynomial elements. 

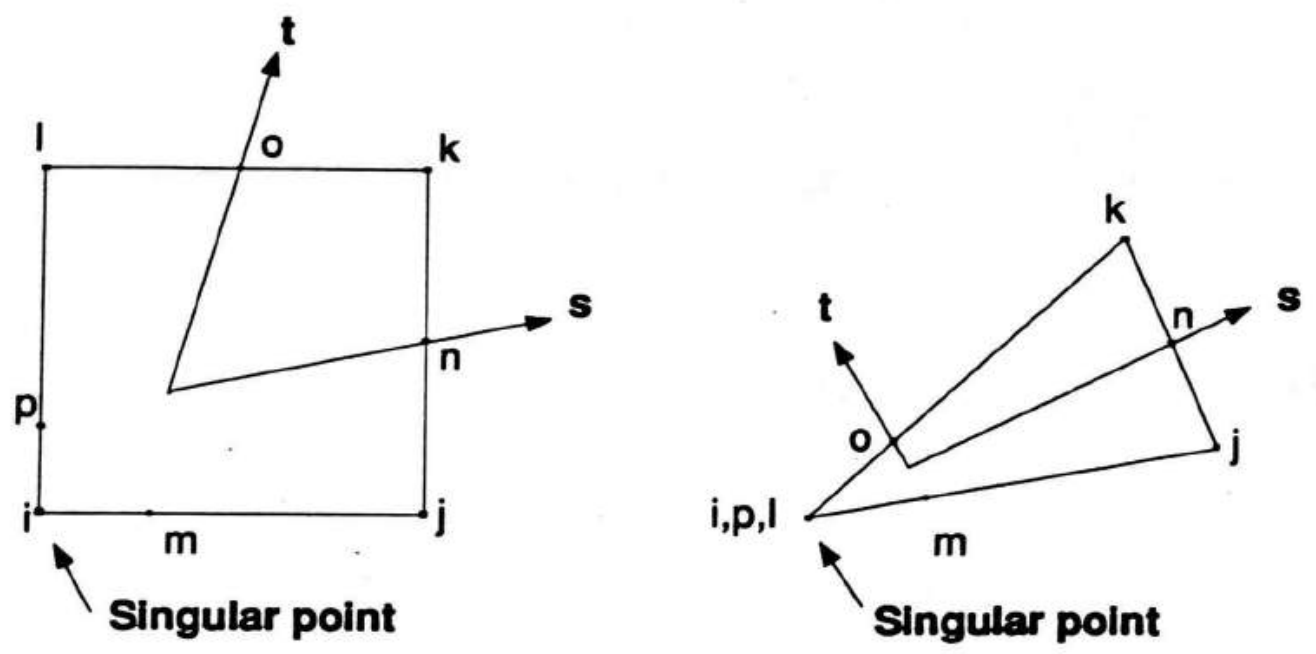

Fig.3.1 Two-dimensional eight-node isoparametric elements: quadrilateral and the same element with three collapsed nodes.

It has been demonstrated by Barsoum [22], that quadratic isoparametric elements with midside nodes adjacent to the crack tip placed at the quarter points, not only contain the inverse square root singularity but also the rigid body motion and constant strain modes. This type of element thus provides a very useful tool for modeling two or three dimensional cracks. Following the notation in Reference [22], the square root singularity for a eight-node plane isoparametric element shown in Fig.3.1, will be derived for the sake of completeness. 
For each element, interior coordinates ( $x$ and $y)$ are related to nodal coordinates $x_{i}$ and $y_{i}$ by:

$$
\begin{aligned}
& x=N_{i} x_{i}+N_{j} x_{j}+N_{k} x_{k}+\ldots N_{p} x_{p} \\
& y=N_{i} y_{i}+N_{j} y_{j}+N_{k} y_{k}+\ldots N_{p} y_{p}
\end{aligned}
$$

Here $N_{I}$ are the shape functions associated with the node I,

$$
\begin{array}{ll}
N_{I}=\left(I+s s_{l}\right)\left(I+t t_{I}\right)\left(s s_{I}+t t_{l}-I\right) / 4 & I=i, j, k, l \\
N_{I}=s_{I}{ }^{2}\left(1+s s_{l}\right)\left(I-t^{2}\right) / 2+t_{I}{ }^{2}\left(I+t t_{I}\right)\left(I-s^{2}\right) / 2 & I=m, n, o, p
\end{array}
$$

$s$ and $t$ are serendipity coordinates (see Fig.3.1), $s_{l}$ and $t_{l}$ are equal to \pm 1 for corner nodes and zero for midside nodes. The displacements are defined in similar form:

$$
\begin{aligned}
& u=N_{i} u_{i}+N_{j} u_{j}+N_{k} u_{k}+\ldots N_{p} u_{p} \\
& v=N_{i} v_{i}+N_{j} v_{j}+N_{k} v_{k}+\ldots N_{p} v_{p}
\end{aligned}
$$


Evaluating the shape functions along $i-m-j(t=-1)$ leads to:

$$
\begin{aligned}
& N_{i}=-s(1-s) / 2 \\
& N_{j}=s(1+s) / 2 \\
& N_{m}=\left(1-s^{2}\right)
\end{aligned}
$$

By use of equations (3.7-3.9) in (3.1) and (3.5) leads to:

$$
\begin{aligned}
& x=-s(I-s) x_{i} / 2+s(I+s) x_{j} / 2+\left(I-s^{2}\right) x_{m} \\
& u=-s(I-s) u_{i} / 2+s(I+s) u_{j} / 2+\left(I-s^{2}\right) u_{m}
\end{aligned}
$$

By choosing $x_{i}=0, x_{m}=1 / 4$ and $x_{j}=1$, then (3.10) yields:

$$
x=s(1+s) / 2+\left(I-s^{2}\right) / 4 ;
$$

therefore, a solution for $s$ is 


$$
s=-1+2 \sqrt{x}
$$

The strain in $\mathrm{x}$-direction is:

$$
\epsilon_{x x}=\partial u / \partial x=(\partial u / \partial s)(\partial s / \partial x)
$$

Finally, using (3.13) and (3.12) in (3.14) leads to:

$$
\epsilon_{x x}=-(3-4 \sqrt{x}) u_{i} / 2 \sqrt{x}-(1-4 \sqrt{x}) u_{j} / 2 \sqrt{x}+(2-4 \sqrt{x}) u_{m} / \sqrt{x}
$$

Therefore, the strain is a function of $1 \sqrt{r}$. The same procedure can be applied to similar lines and for three dimensional elements.

\subsection{Numerical Process}

One of the most difficult problems in fracture mechanics is the analysis of cracks in three-dimensional bodies. The intersection of an area crack with a free surface is referred to as a surface flaw. These type of defects are very common in many engineering applications but exact 
solutions of these problems are seldom determined. Investigators have resorted to numerical methods to obtain SIFs in these situations.

To solve this numerical problem using the finite element program ANSYS a substructuring method is used. The analysis is divided in two parts: the model and the submodel.

\subsubsection{The Model}

This part consists of developing a coarse model (see Fig.3.2) which represents the entire structure. Taking advantage of the three planes of symmetry, only a section of the model is analyzed. The angle $\theta$ is function of the number of cracks ( $\Theta=180^{\circ} / \mathrm{n}$ ). The surface crack in the model is shown in Fig.3.2, where $a$ is the crack depth, $c$ is the semi-crack length, $r_{i}$ is the inner radius, $t$ is the wall thickness and $2 L$ is the length of the cylinder. 


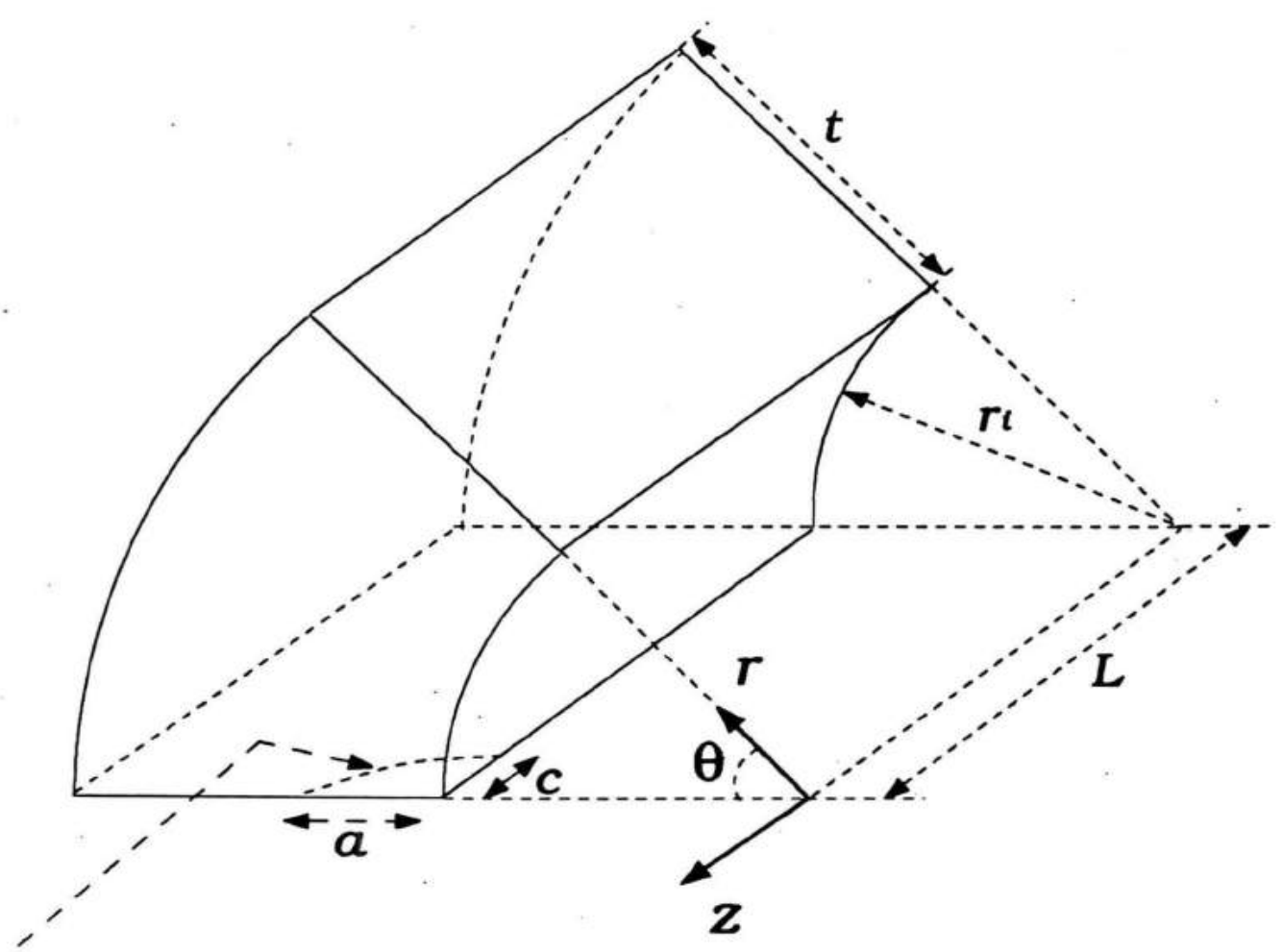

\section{crack front}

Fig.3.2 Semi-elliptical surface crack in a cylinder. The model represents the entire cylinder with three planes of symmetry: $\Theta=0^{\circ}, \Theta=180^{\circ} / n$ and $Z=0$.

The finite element model shown in Fig.3.3 is meshed with ten-node tetrahedral elements (STIF92) and it is generated using the automatic mesh generator. To obtain a better representation of the displacement field, smaller elements are chosen near the crack region, meanwhile far away the element size is larger. 


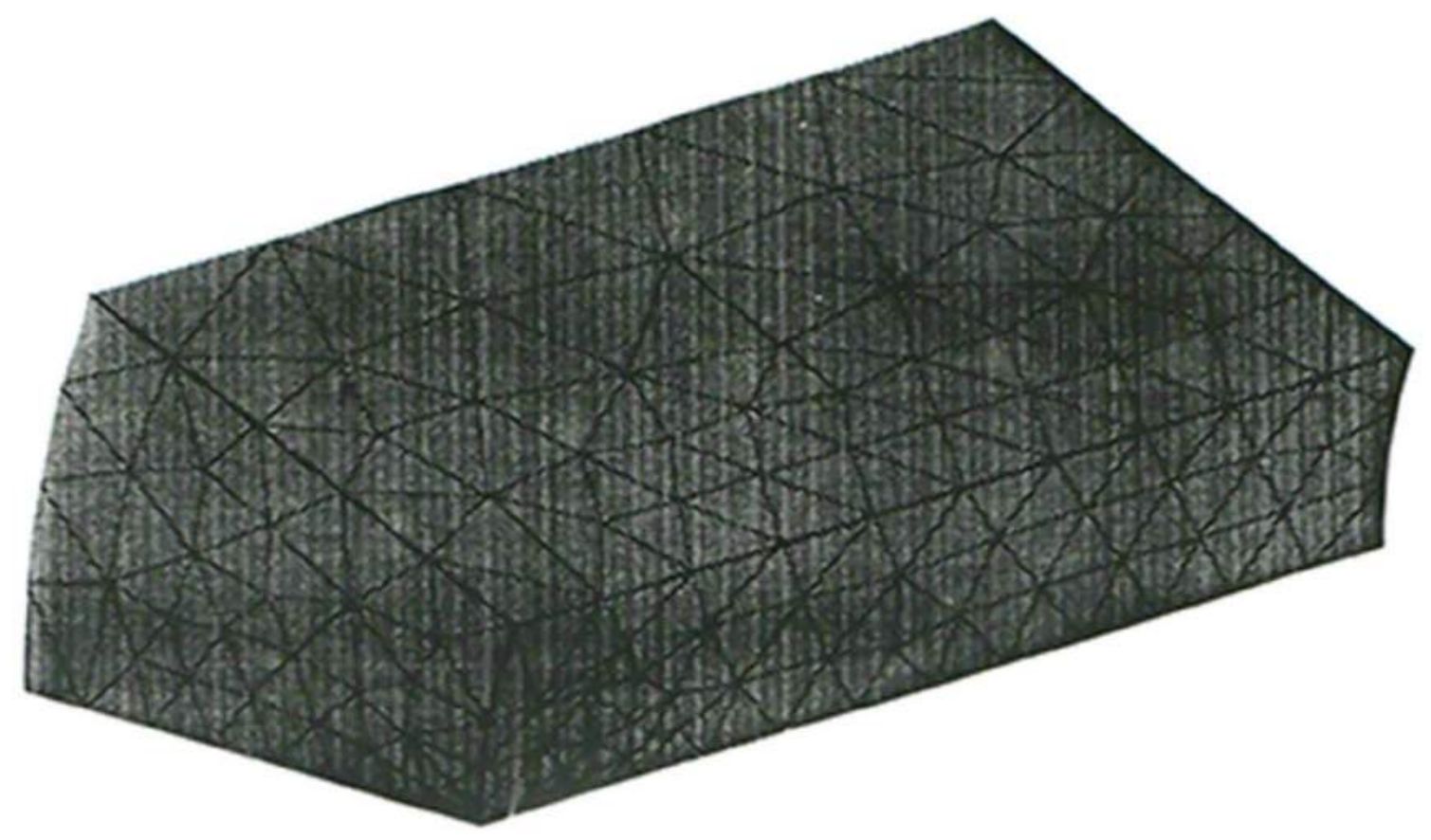

Fig. 3.3 The model meshed with 10-node tetrahedral elements.

The constant pressure load is applied perpendicular to the internal surface of the cylinder and on the crack face. Symmetry boundary conditions are applied on the corresponding planes of symmetry. In order to approximate an infinite cylinder and avoid the end-effect of the cylinder the ratio of length of the cylinder to the crack length. $L / c$. is taken as 10 . Poisson's ratio is assumed to be 0.3 , representative of metal cylinders. 
Nevertheless, this model is considered to be inadequate for local crack-front analysis; therefore a submodel is created.

\subsubsection{The Submodel}

To generate the submodel, direct meshing is used and 20-node solid elements surround the entire crack-front. By collapsing four nodes and adjusting midside nodes, singular elements are positioned along the crackfront as shown in Fig.3.4. The radial size of the submodel is $2 a / 3$ for circular cracks of radius a and varies from $2 c / 3$ to $2 a / 3$ for elliptical cracks, where $\mathrm{c}$ is the semi-length and a is the depth of the crack. Therefore most of the crack is included in the submodel. The singular element size is a/8 wherein eight elements are positioned around the crack front and evenly spaced every nine degrees in the $\phi$ direction as shown in Fig.3.5.

This direct generation approach gives better control of the local crackfront mesh, allows the use of singular elements, and makes the extraction of $K_{I}$ easier. 


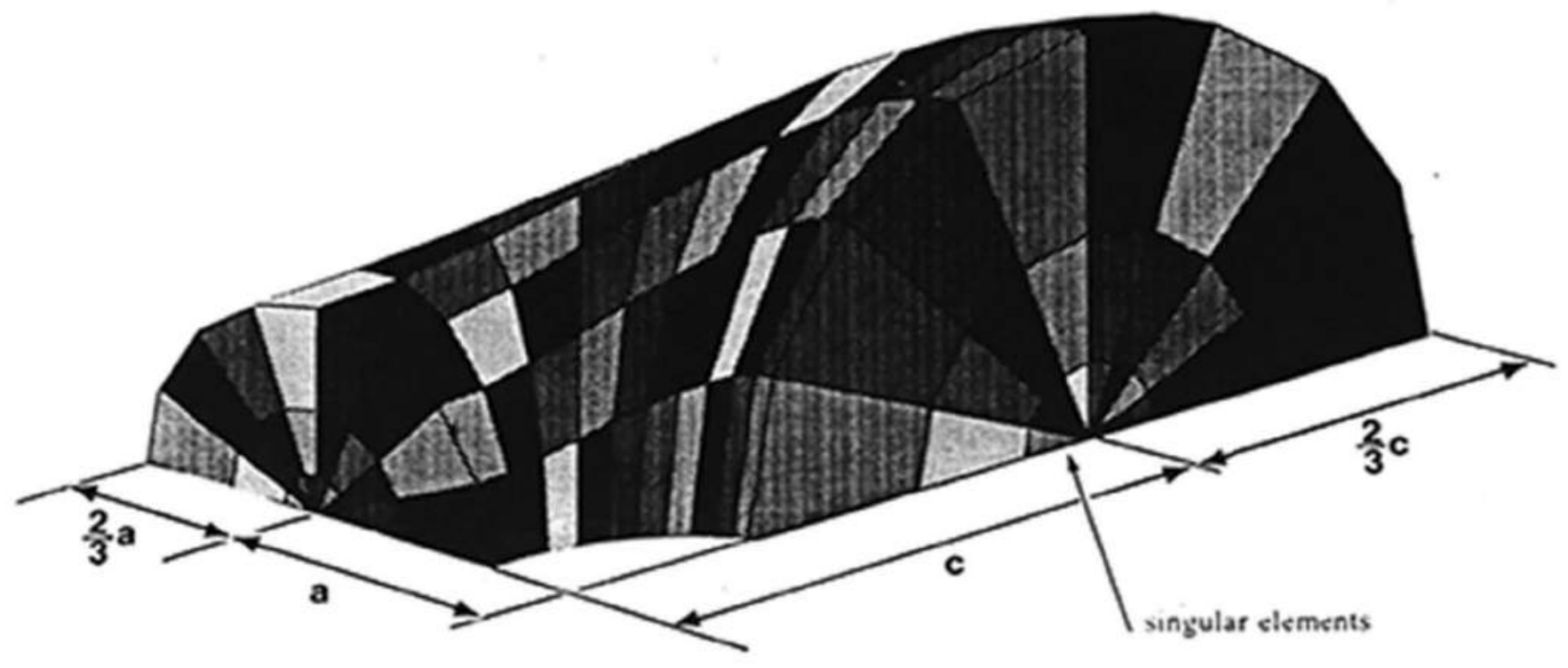

Fig.3.4 The submodel meshed with 20-node solid elements.

As shown in Fig.3.5. for elliptical cracks the angle $\phi$ is measured as in Raju and Newman [12]. The SIF for these cases are obtained in planes perpendicular to the crack-front. 


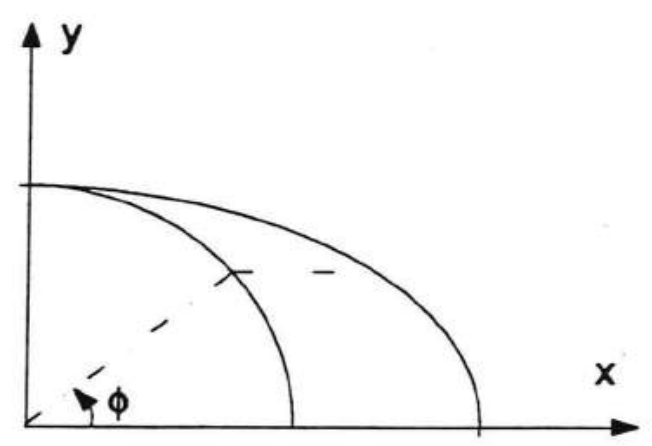

Fig.3.5 Measure of the angle $\phi$ for semi-elliptical cracks.

\subsubsection{Substructuring Procedure}

After the model is generated, meshed and the boundary conditions applied, the problem is solved for the nodal displacements. Once the nodal displacements are obtained, the submodel around the crack front is created using direct generation. The resulting displacements from the model are applied to the outer boundary of the submodel using the AUX1 cut-boundary procedure. Finally, the submodel is solved for its displacements after applying the boundary conditions. Utilizing the KCALC command the stress intensity factor for ten points along the crack-front is computed. 
The direct generation of the nodes and elements in the submodel is a difficult process which demands extensive time and effort. Since, for all the cases to be solved, a submodel is generated modifying only the crack-depth, $a$, and semi crack-length, $c$, a macro was created which generated the nodes and elements easily and without errors. This macro is a user-defined sequence of data input commands written to an external file that may be used at any other input location. Also, the macro may contain parameters which may be assigned values by the user. The execution may be repeated with the parameters incremented. The macros created to generate the submodel are listed in Appendix C.

\subsubsection{Extraction of stress intensity factor}

Although there are many methods to estimated the SIF from a finite element solution, the most utilized are : (1) the displacement method, (2) the nodal force method and (3) the line integral (energy )method. All these methods estimate the SIF using established crack tip relations and are described in reference [23]. Since the calculation of the SIF based on the displacement method is made automatically in ANSYS using the command 
KCALC, only this method is reviewed here.

The displacements and stresses in the vicinity of the crack are related to the three modes and are expressed in the crack front coordinates of the Fig. 3.6 by $[24]$ as,

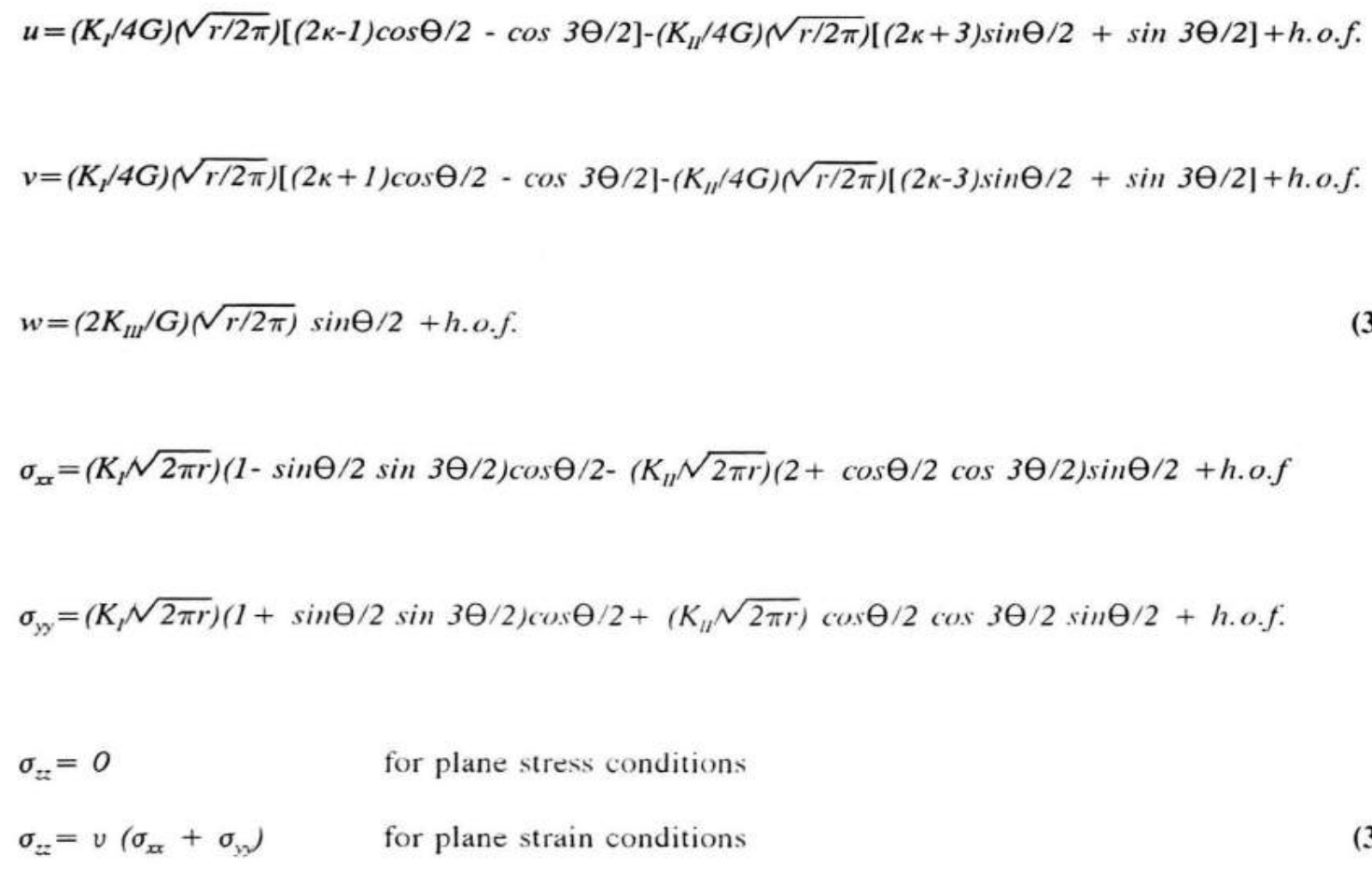

where $u, v$ and $w$ are the displacements in rectangular coordinate system; $K_{l}$, $K_{I I}$ and $K_{I I I}$ are the stress intensity factors in mode I, II and III respectively; $\sigma_{x x}, \sigma_{y y}$ and $\sigma_{z z}$ are the normal stress components in rectangular coordinate system; $r$ and $\Theta$ are local polar coordinate system; $G$ is the shear modulus; 
is Poisson's ratio; h.o.f. are higher order factors; and the variable $\kappa$ is a conversion factor between plane strain and plane stress and is expressed as,

$$
\begin{array}{ll}
\kappa=3-4 v & \text { for plane strain } \\
\kappa=(3-v) /(1+v) & \text { for plane stress }
\end{array}
$$

Neglecting higher order terms as $r \rightarrow 0$ the displacement equations give,

$$
\begin{aligned}
& u=\left(K_{I I} / 2 G\right)(\sqrt{r / 2 \pi})(I+\kappa) \\
& v=\left(K_{I} / 2 G\right)(\sqrt{r / 2 \pi})(I+\kappa) \\
& w=\left(K_{I I I} / G\right)(\sqrt{r / 2 \pi})
\end{aligned}
$$

To extract the values of $K_{I}$, the finite element results are compared with equations (3.19). To calculate $K_{l}$ from mode I displacements, the behavior of $v(r)$ is approximate by,

$$
v^{\prime} / \sqrt{r}=A+B r
$$


where $v^{\prime}$ is the finite element approximation to the crack-face displacement, $v$, and $A$ and $B$ are constants determined from a linear curve fit of nodal displacements. Figure 3.6 shows that three nodes $(i, j, k)$ are needed for a half-crack model, all the displacements are relative to crack-tip node $i$. Once $A$ and $B$ are determined, the limit $r \rightarrow 0$ is taken, yielding

$$
\lim _{r \rightarrow O} v^{\prime} / \sqrt{r}=A
$$

From (3.19) and (3.21),

$$
K_{I}=(2 G A \sqrt{2 \pi}) /(I+\kappa)
$$

The method of substructuring explained above was validated solving a similar case but for only two cracks and $t / r_{i}=0.25$. This problem, as mentioned before was solved by Raju and Newman [12] using the nodalforce method. The results of this validation are presented in the next chapter. 


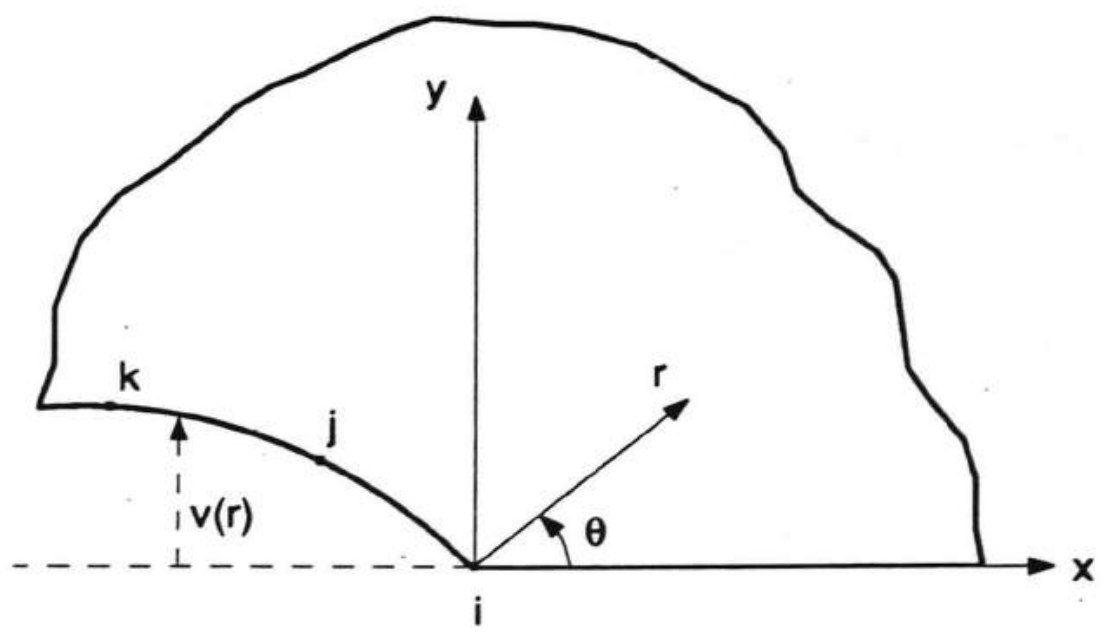

Fig.3.6 Nodes used for the crack-front displacements. 


\subsection{Maximum stress intensity factor equation}

An empirical equation was developed in the present study to evaluate the maximum stress intensity factor for different values of $a / c, a / t, n, p, r_{i}$ and $t$. A manner similar to Raju and Newman [12], the coefficients of this equation were determined by nonlinear fitting. However, the equation developed herein applies not only for two cracks, but for different number of cracks. The empirical equation is determined by a combination of two effects:(1) the function $f_{l}$, which describes the effect of the crack depth to the wall thickness of the cylinder, $a / t$. This expression is similar to that one used in [12] and is written as,

$$
f_{1}=b_{1}+b_{2}\left(\frac{a}{t}\right)+b_{3}\left(\frac{a}{t}\right)^{2}+b_{4}\left(\frac{a}{t}\right)^{3}
$$

(2) the function $f_{2}$, which describes the effect of the separation between cracks and was utilized by Perl and Aroné [7] in the two dimensional problem to describe the effect of the number of cracks. This expression is 
given by,

$$
f_{2}=1+\frac{b_{7}}{1+\frac{2 c}{d}}+\frac{b_{8}}{\left(1+\frac{2 c}{d}\right)^{2}}+\frac{b_{9}}{\left(1+\frac{2 c}{d}\right)^{3}}
$$

Here, $d$ is the distance between two adjacent cracks at the deepest point. The ratio $2 c / d$ takes into account the effect of the crack length to the separation between cracks and is written in terms of $a / t$ and $n$, as,

$$
\frac{2 c}{d}=\frac{b_{5}\left(\frac{a}{t}\right) n}{1+b_{6}\left(\frac{a}{t}\right)}
$$

The empirical equation was obtained by superposition of the two effects of the functions $f_{1}$ (3.23) and $f_{2}$ (3.24) and was fitted to the finite element results to obtain the coefficients $b_{i}$ for fixed values of $a / c$. This equation evaluates the maximum stress intensity factor for multiple radial cracks in pressurized cylinders and is given by, 


$$
\frac{K_{I}}{\frac{p \cdot r_{i}}{t} \sqrt{\frac{\pi \cdot a}{Q}}}=\frac{t}{r_{i}}\left(\frac{r_{o}^{2}}{r_{o}^{2}-r_{i}^{2}}\right) \cdot f_{1} \cdot f_{2}
$$

where $p$ is the internal pressure, $r_{i}$ is the inner radius, $r_{o}$ is the outer radius, $t$ the wall thickness of the cylinder and $Q$ is the shape function for an ellipse. The factor $Q$ is given by [12],

$$
Q=1+1.464\left(\frac{a}{c}\right)^{1.65}
$$

Because of the limitation in the wave front in our version of ANSYS, it was not possible to obtain a solution for cases where $a / t \leq 0.05$, therefore we were unable to compare the results obtained by equation (3.26) with the limiting case of infinite wall thickness $(a / t \rightarrow 0)$. 


\section{Chapter 4. Results}

For all the solutions presented in the following sections, a normalization factor $\left(C_{o}\right)$ is used, which is obtained from reference [12] and is defined as:

$$
C_{o}=\left(\frac{p \cdot r_{i}}{t}\right) \sqrt{\frac{\pi \cdot a}{Q}}
$$

Here, $p$ is the constant pressure applied in the cylinder, $r_{i}$ is the inner radius of the cylinder, $t$ is the wall thickness, $a$ is the crack-depth and $Q$ is the shape factor for an ellipse (equation 3.27). The length of the cylinder was always large enough $(L / C=10)$ to avoid the end effect on the results.

\subsection{Validation of the model}

In this section, a comparison between the present results and solutions obtained by Raju and Newman [12] are presented. These solutions correspond to a pressurized cylinder having two cracks, where the ratio of the wall thickness to the inner radius, $t / r_{i}$ is 0.25 subject to two different 
crack configurations.

1. Semi-circular surface crack

Figure 4.1 shows the mode I stress intensity factor for two diametrically located semi-circular cracks $(a / c=1)$, in a cylinder of crack depth to the wall thickness ratio $a / t=0.2$ with the ratio of the wall thickness to the inner radius of $t / r_{i}=0.25$. The maximum difference between the present results and those obtained in [12] is $4.3 \%$ lower, and, it occurs at $\phi=22.5^{\circ}$.

2. Semi-elliptical surface crack

Figure 4.2 shows the variation of mode I stress intensity factor for two semi-elliptical cracks $(a / c=0.4)$ in a cylinder with $a / t=0.2$ and $t / r_{i}=0.25$. The maximum difference between the present results and those obtained in reference [12] is $4.4 \%$ lower, and, it occurs at the deepest point $\left(\phi=90^{\circ}\right)$.

The present results were also compared with the finite element analysis of Kirkhope [13], the results of the present study were $8 \%$ lower. These comparisons show that the present results are in very good agreement. 


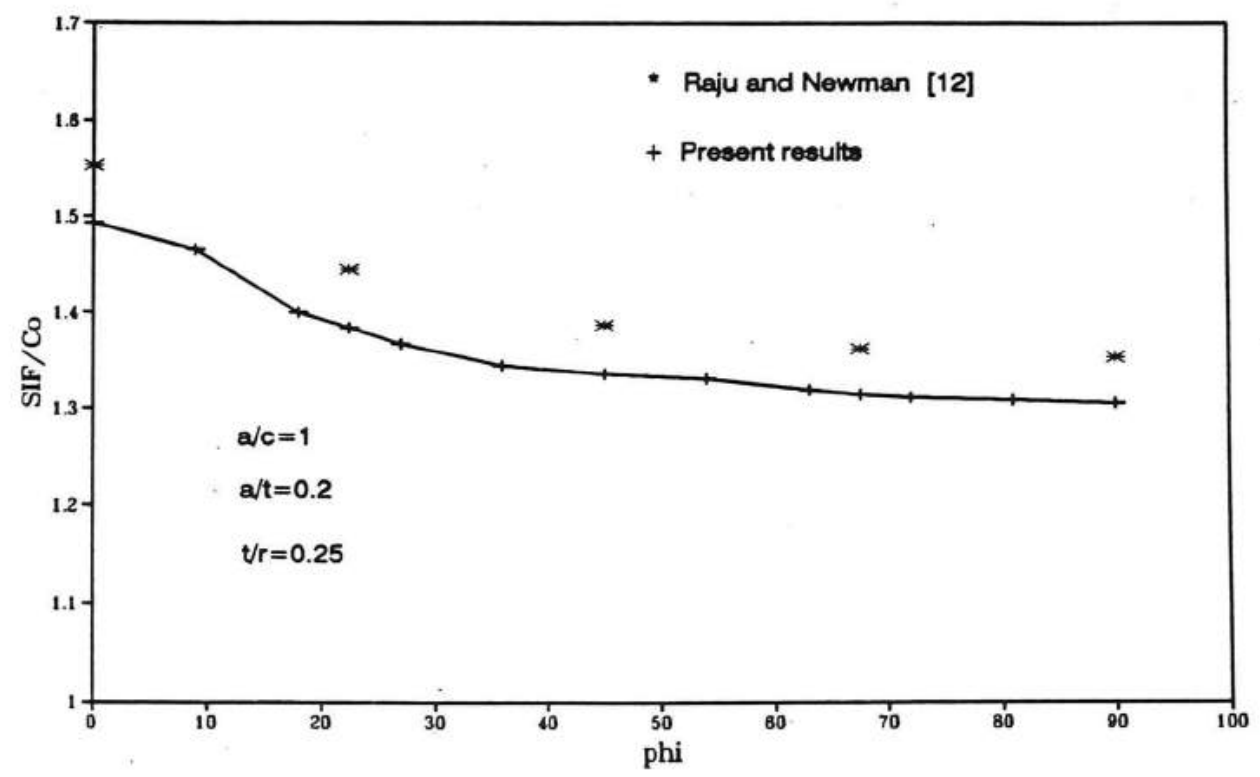

Fig.4.1 Comparison of stress intensity factor along crack-front for two semi-circular surface crack in pressurized cylindrical vessel.

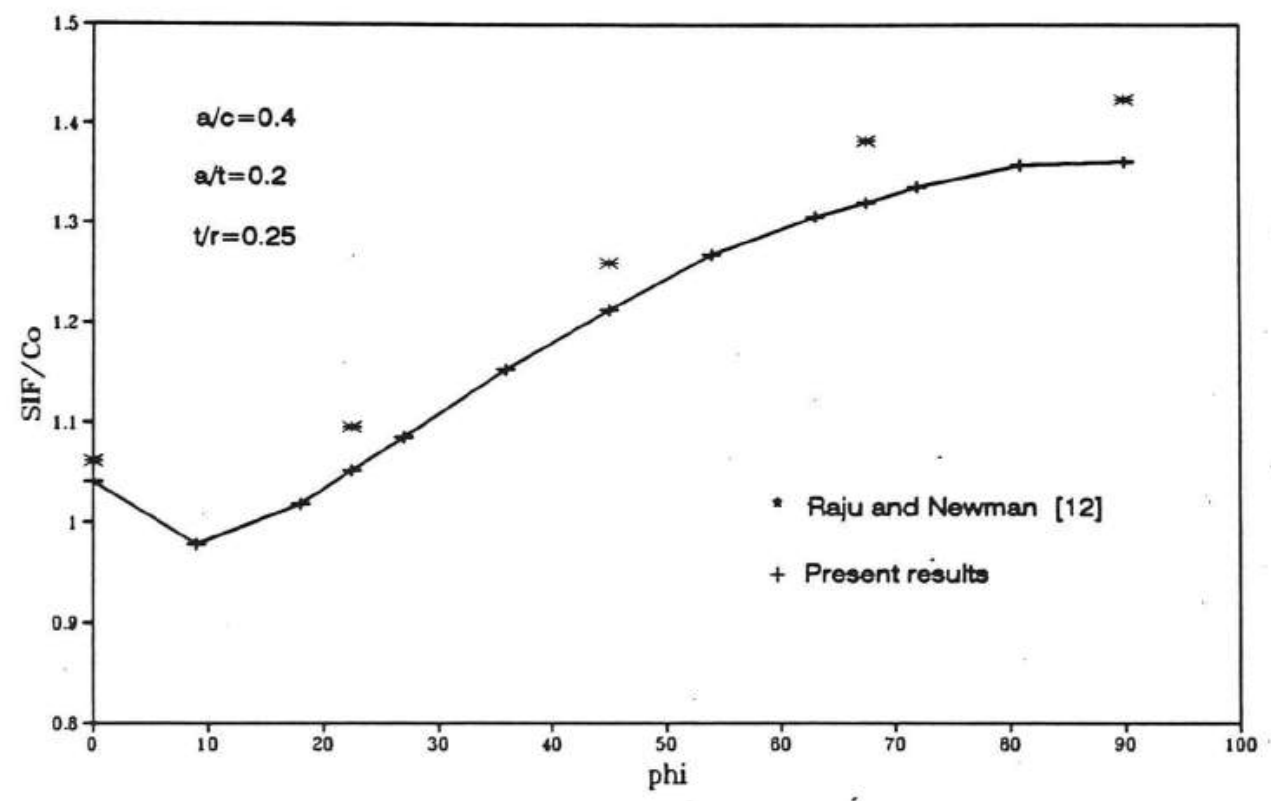

Fig.4.2 Comparison of stress intensity factor along crack-front for two semi-elliptical surface crack in pressurized cylindrical vessel. 
Nevertheless, it must be stressed that the solutions in $[12,13]$ were obtained using a nodal force method to evaluate the SIF which gives upper bound solutions [25]. Herein, the present results are determined employing the displacement method which gives lower bound for the true value [25].

In order to estimate the number of elements necessary to achieve convergence in the finite element mesh, a convergence analysis was done. In Fig.4.3, the displacements in $y$-direction of the node located at $x=1$, $y=0$, and $z=-0.175$ are shown as a function of the number of elements. For 1100 elements or more the displacements converged.

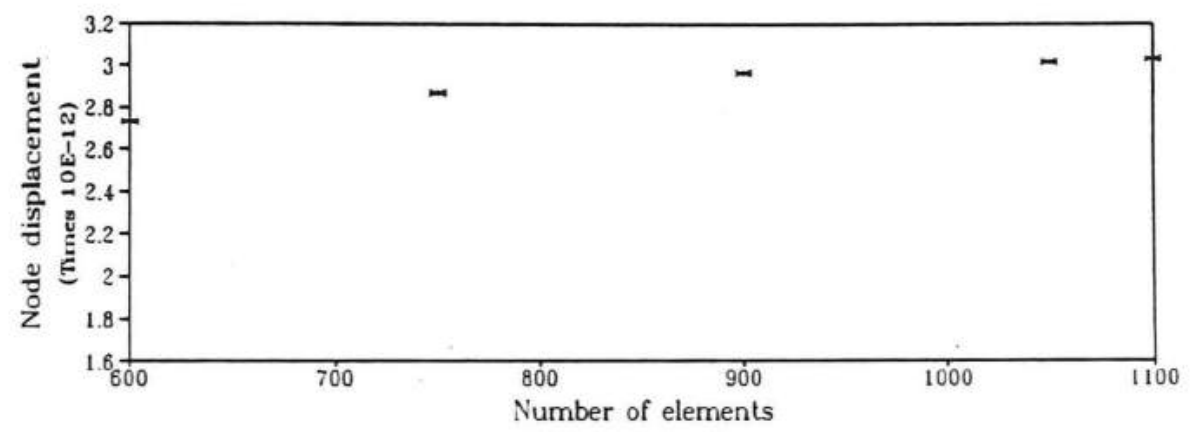

Figure 4.3 Number of elements necessary to achieve convergence.

The above considerations demonstrated that the modeling and analysis procedure utilized in this investigation offer accurate results thereby validating the solution obtained for multiple configurations of cracked cylinders presented in the following section. 


\subsection{Results}

Table 4.1 shows the cases solved in this study for different crack shapes: the ellipticity of the crack, $a / c=0.2,0.5,0.1$, and 1.5 ; the ratio of crack depth to the wall thickness, $a / t=0.05,0.1,0.2,0.4$, and 0.6 ; and the number of cracks, $n=1,2,4, \ldots, n \max$. The quantity nmax depends upon the factor $2 c / 3$. This factor is very important, because the submodel has to cover $2 / 3$ of the crack to obtain more accurate results. To increase the maximum number of cracks, some cases were solved using $c / 3$ and $c / 2$ instead of $2 c / 3$ (see Table 4.1). These cases were compared to previously solved problems and the error in $K$, was found to be $2 \%$ to $3 \%$.

In appendix $\mathrm{A}$, the normalized mode I stress intensity factors along the crack front for all the cases solved in this study, are presented. In appendix $\mathrm{B}$, the results are plotted on graphs showing the variation of the stress intensity factors for different crack configurations as functions of the angle $\phi$, and the number of cracks, $n$. 
Table 4.1 Crack configurations solved in the present study

\begin{tabular}{||c||c||l||}
\hline $\mathrm{a} / \mathrm{c}$ & $\mathrm{a} / \mathrm{t}$ & \multicolumn{1}{|c|}{$\mathrm{n}$} \\
\hline 0.2 & 0.05 & $1 ; 2 ; 4 ; 8 ; 16 ; 32 * *$ \\
\hline & 0.1 & $2 ; 4 ; 8 ; 16 * *$ \\
\hline & 0.15 & $2 ; 4 ; 6 ; 12$ \\
\hline & 0.2 & $1 ; 2 ; 4 ; 8$ \\
\hline \hline 0.5 & 0.05 & $1 ; 2 ; 4 ; 8 ; 16 ; 32 * ; 45 ; 64^{*} ; 90^{* *}$ \\
\hline & 0.1 & $1 ; 2 ; 4 ; 8 ; 16 ; 20 ; 32 ; 40^{* *}$ \\
\hline & 0.15 & $1 ; 2 ; 4 ; 8 ; 16 * ; 32 * *$ \\
\hline & 0.2 & $1 ; 2 ; 4 ; 8 ; 16 * ; 20^{* *}$ \\
\hline & 0.4 & $1 ; 2 ; 4 ; 8 * *$ \\
\hline & 0.6 & $1 ; 2 ; 3 ; 6 * *$ \\
\hline \hline & 0.05 & $1 ; 2 ; 4 ; 8 ; 16 ; 32 ; 64$ \\
\hline & 0.1 & $1 ; 2 ; 4 ; 8 ; 16 ; 32 ; 45$ \\
\hline & 0.15 & $1 ; 2 ; 4 ; 8 ; 16 ; 32$ \\
\hline & 0.2 & $1 ; 2 ; 4 ; 8 ; 16 ; 20$ \\
\hline & 0.4 & $1 ; 2 ; 4 ; 8 ; 10$ \\
\hline & 0.6 & $1 ; 2 ; 4 ; 6$ \\
\hline \hline 1.5 & 0.05 & $1 ; 2 ; 4 ; 8 ; 16 ; 32 ; 45 ; 64 ; 90 ; 180^{* *}$ \\
\hline & 0.1 & $1 ; 2 ; 4 ; 8 ; 16 ; 32 ; 50 ; 64^{*} ; 100^{* *}$ \\
\hline & 0.15 & $1 ; 2 ; 4 ; 8 ; 16 ; 32 ; 64 * *$ \\
\hline & 0.2 & $1 ; 2 ; 4 ; 8 ; 16 ; 25 ; 32 * ; 50^{* *}$ \\
\hline & 0.4 & $1 ; 2 ; 4 ; 8 ; 16 * ; 32 * *$ \\
\hline & 0.6 & $1 ; 2 ; 4 ; 8 ; ; 10 ; 16 * ; 20^{* *}$ \\
\hline
\end{tabular}

* $c / 2$ submodel size

** $c / 3$ submodel size 


\subsection{Empirical equation results}

As was explained in chapter 3, an empirical equation was developed in this study to estimate the maximum stress intensity factor in a pressurized multicracked cylinder. The coefficients (see table 4.2 ) were determined by nonlinear fitting of the finite element results and were found for fixed values of $a / c$. The variation of $a / c$ is included in the parameters $b_{i}$.

Table 4.2 Values of the coefficients $b_{i}$ (equation 3.26)

\begin{tabular}{|c|c|c|c|c|c|c|c|c|c||}
\hline$a / c$ & $b_{1}$ & $b_{2}$ & $b_{3}$ & $b_{4}$ & $b_{5}$ & $b_{6}$ & $b_{7}$ & $b_{8}$ & $b_{9}$ \\
\hline \hline 0.2 & 0.43819 & -0.74219 & 4.30884 & -9.32273 & 0.64230 & 1.85949 & 3.74752 & 5.82288 & -6.22339 \\
\hline 0.5 & 0.63322 & -0.80852 & 1.53499 & -0.65347 & 0.84945 & 4.23347 & 3.52572 & 1.44541 & -2.98802 \\
\hline 1.0 & 0.40548 & -0.25485 & 0.45425 & -0.15454 & 0.34017 & 1.82339 & 4.76818 & 6.46741 & -7.02430 \\
\hline 1.5 & 0.50172 & -0.54225 & 1.29890 & -0.98514 & 0.37996 & 1.14850 & 7.22075 & 1.83183 & -5.00655 \\
\hline \hline
\end{tabular}

Appendix D shows the maximum SIF obtained using the empirical equation (3.26). When compared with the finite element results, the maximum error was found to be $\pm 5.7 \%$. Equation (3.26) is valid for $t / r_{i}$ close to 1 ; a comparison of the results obtained by this equation and those obtained in [12] is not possible because results in [12] are obtained for $t / r_{i} \leq 0.25$ 


\section{Chapter 5. Analysis and Discussion}

In the following sections representative graphs are analyzed and discussed, the graphs and tables of all the cases solved in this study are presented in the Appendices A and B.

\subsection{Semi-circular surface cracks $(a / c=1)$}

Figures 5.1, 5.2, and 5.3 show the variation of the SIF for semicircular surface cracks $(a / c=1)$ for ratios of $a / t=0.05,0.2$, and 0.6 . These results are given as a function of the angle, $\phi$, and the number of cracks, $n$.

- The case of two cracks gives the maximum SIF, for all the cases. As the number of cracks increases, the SIF decreases tending to a constant value at the deepest point of the $\operatorname{crack}\left(\phi=90^{\circ}\right)$.

- The percentage difference in SIF between the $n=2$ case and the $n=1$ case is $0.3 \%$ for small cracks $(a / t=0.05)$. This difference increases with increasing crack size, with a maximum percentage difference of $3.6 \%$ for $a / t=0.6$. For the smallest crack $(a / t=0.05)$, even the $n=8$ case gives a 


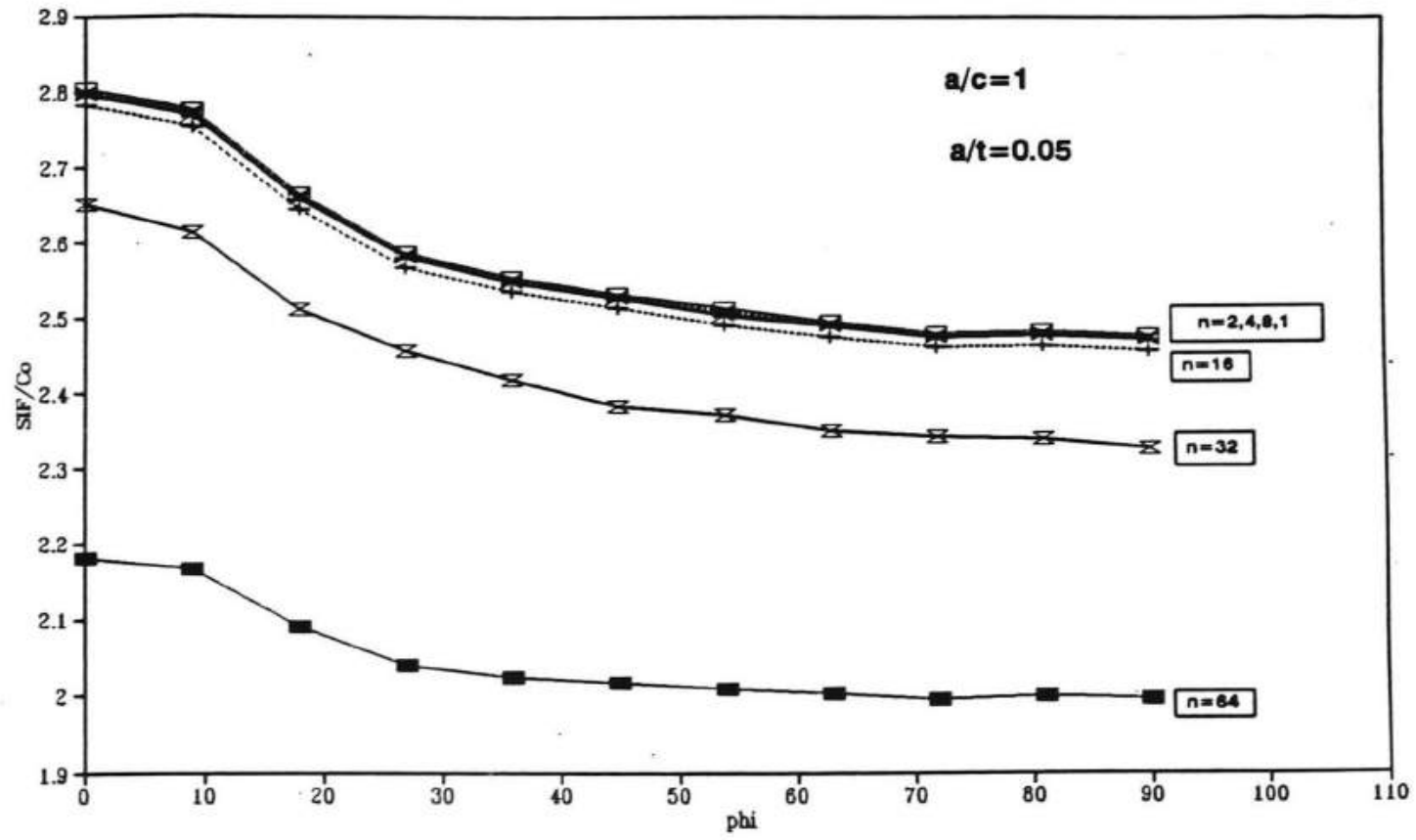

Figure 5.1 Stress intensity factor along crack-front for semi-circular surface crack in pressurized cylindrical vessel $(a / t=0.05)$

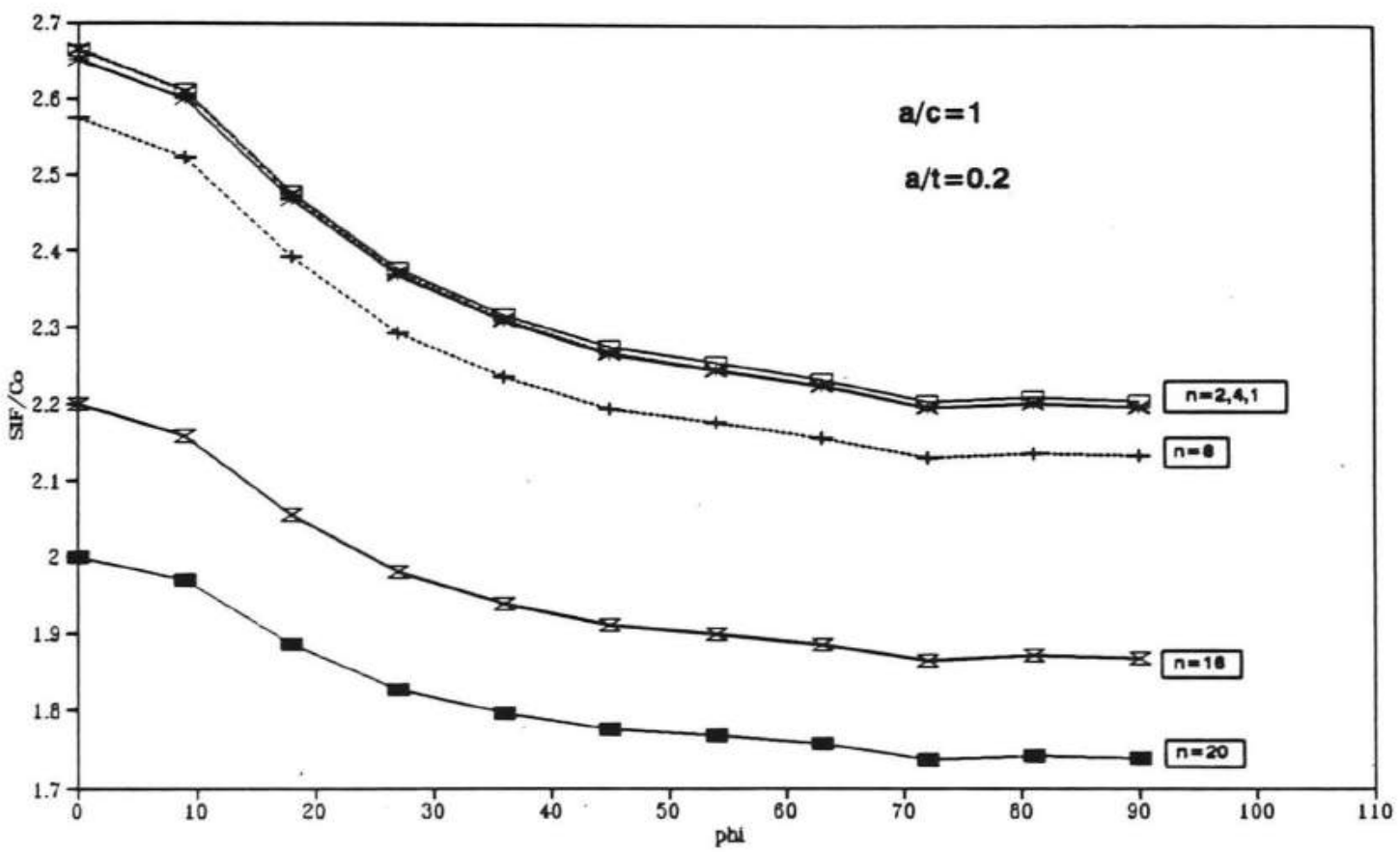

Fig.5.2 Stress intensity factor along crack-front for semi-circular surface crack in pressurized cylindrical vessel $(a / t=0.2)$. 


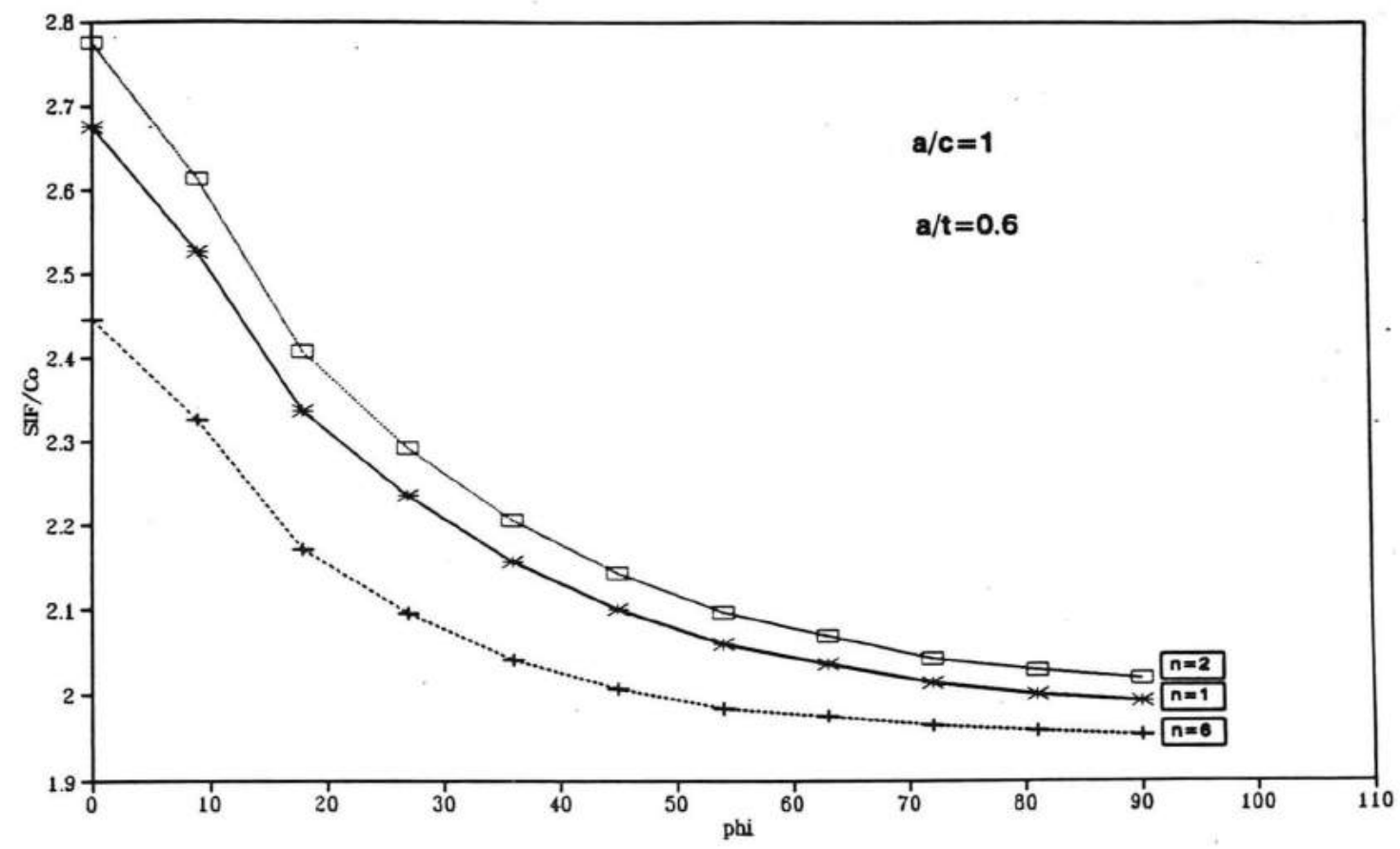

Figure 5.3 Stress intensity factor along crack-front for semi-circular surface crack in pressurized cylindrical vessel $(a / t=0.6)$

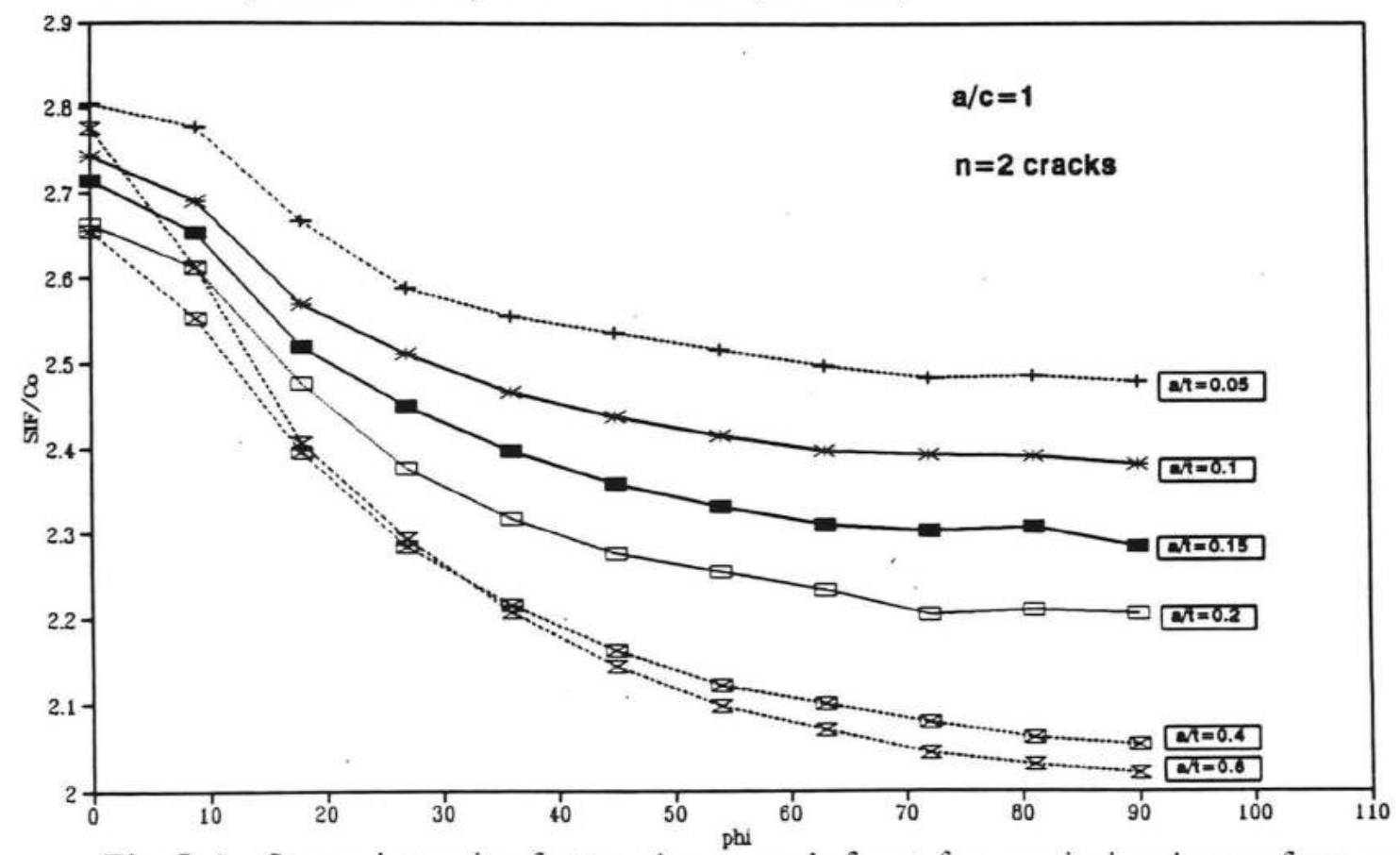

Fig.5.4 Stress intensity factor along crack-front for semi-circular surface crack in pressurized cylindrical vessel 
higher SIF than the single crack case.

- The maximum value of the SIF for a fixed $a / t$ is at the intersection of the crack and the surface $\left(\phi=0^{\circ}\right)$. Then the SIF decreases more rapidly as the size of the crack $(a / t)$ increases. This, also, can be observed in Fig.5.4.

Figure 5.4 shows the variation of the SIF for two semi-circular cracks $(a / c=1)$ and various crack size ratios, $a / t=0.05,0.1,0.15,0.2,0.4$, and 0.6 as a function of the angle $\phi$. For $a / t \leq 0.4$ the normalized SIF is larger for smaller cracks.

Figure 5.5 shows the variation of the maximum $\operatorname{SIF}\left(\phi=0^{\circ}\right)$ for semicircular cracks $(a / c=1)$ as a function of the number of cracks and the crack depth $(a / t)$. For small cracks (small $a / t)$ the values of the SIF are nearly the same but this difference increases with the increasing of $a / t$. 


\subsection{Semi-elliptical surface cracks.}

\subsubsection{Ellipticity of the crack less than one $(a / c<1)$}

Figures 5.6, 5.7, and 5.8 show the variation of the SIF for semielliptical surface cracks $(a / c=0.5)$ and the ratio $a / t=0.05,0.2$, and 0.6 . These results are given as a function of the angle, $\phi$, and the number of cracks, $n$.

- The case of two cracks gives the maximum SIF, for all the cases.

- The percentage difference in the stress intensity factor between the $n=2$ case and the $n=1$ case is $0.2 \%$ for small cracks $(a / t=0.05)$. This difference increases with increasing crack size, reaching a maximum of 5.7 $\%$ for $a / t=0.6$

- The maximum SIF occurs at the deepest point of the $\operatorname{crack}\left(\phi=90^{\circ}\right)$ for $a / t \leq 0.4$. The SIF increases as the depth of the crack $(a / t)$ increases. For $a / t=0.6$, the maximum SIF is at $\phi=0^{\circ}$.

- The minimum SIF for small cracks $(a / t \leq 0.1)$ occurs at approximately $\phi=9^{\circ}$. As the crack depth increases, the angle for minimum SIF increases to $\phi=27^{\circ}$ for $0.2 \leq a / t \leq 0.6$.

- As the number of cracks increases, the difference between the 


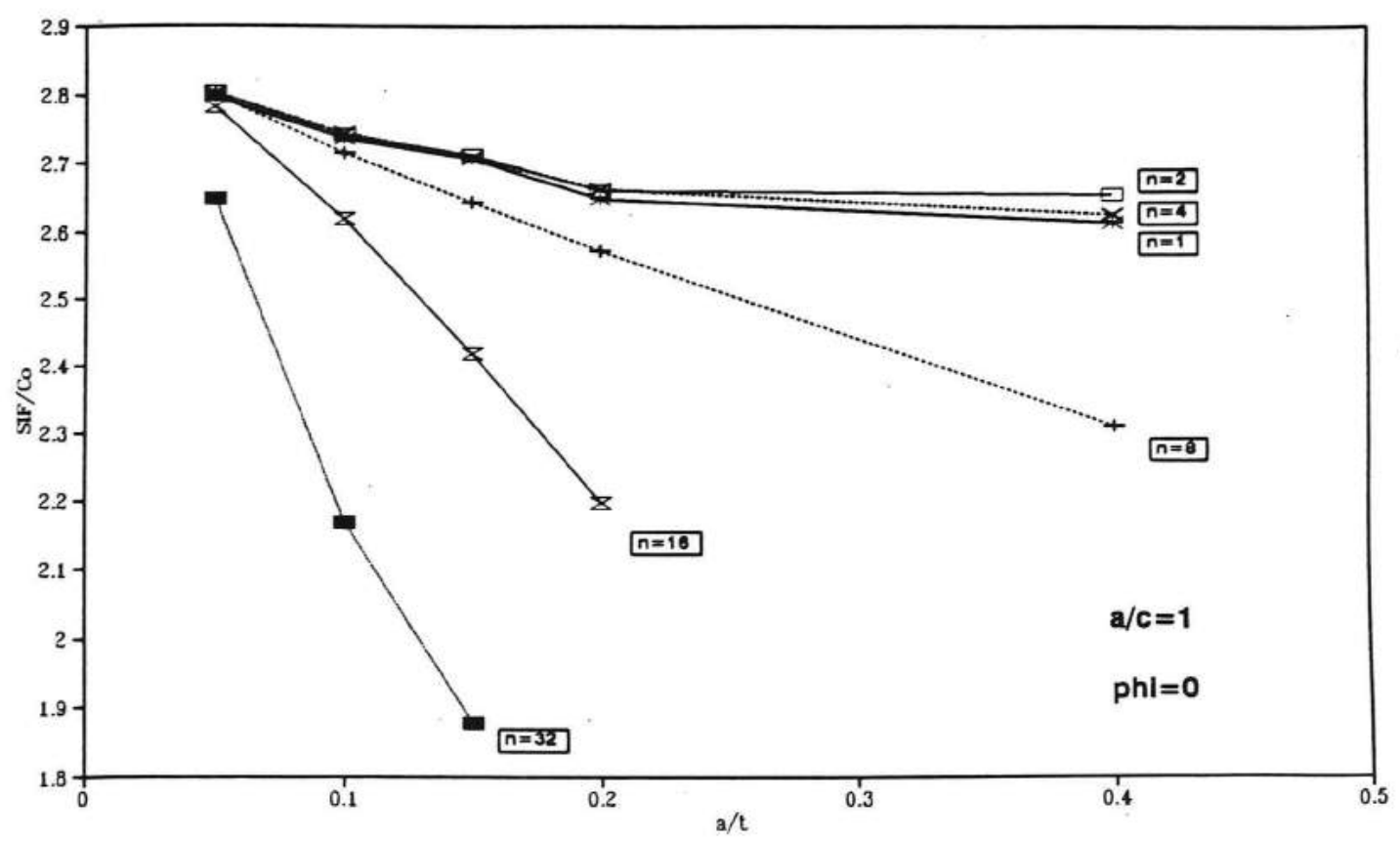

Fig.5.5 Maximum stress intensity factor along crack-front for semi-circular surface crack in pressurized cylindrical vessel.

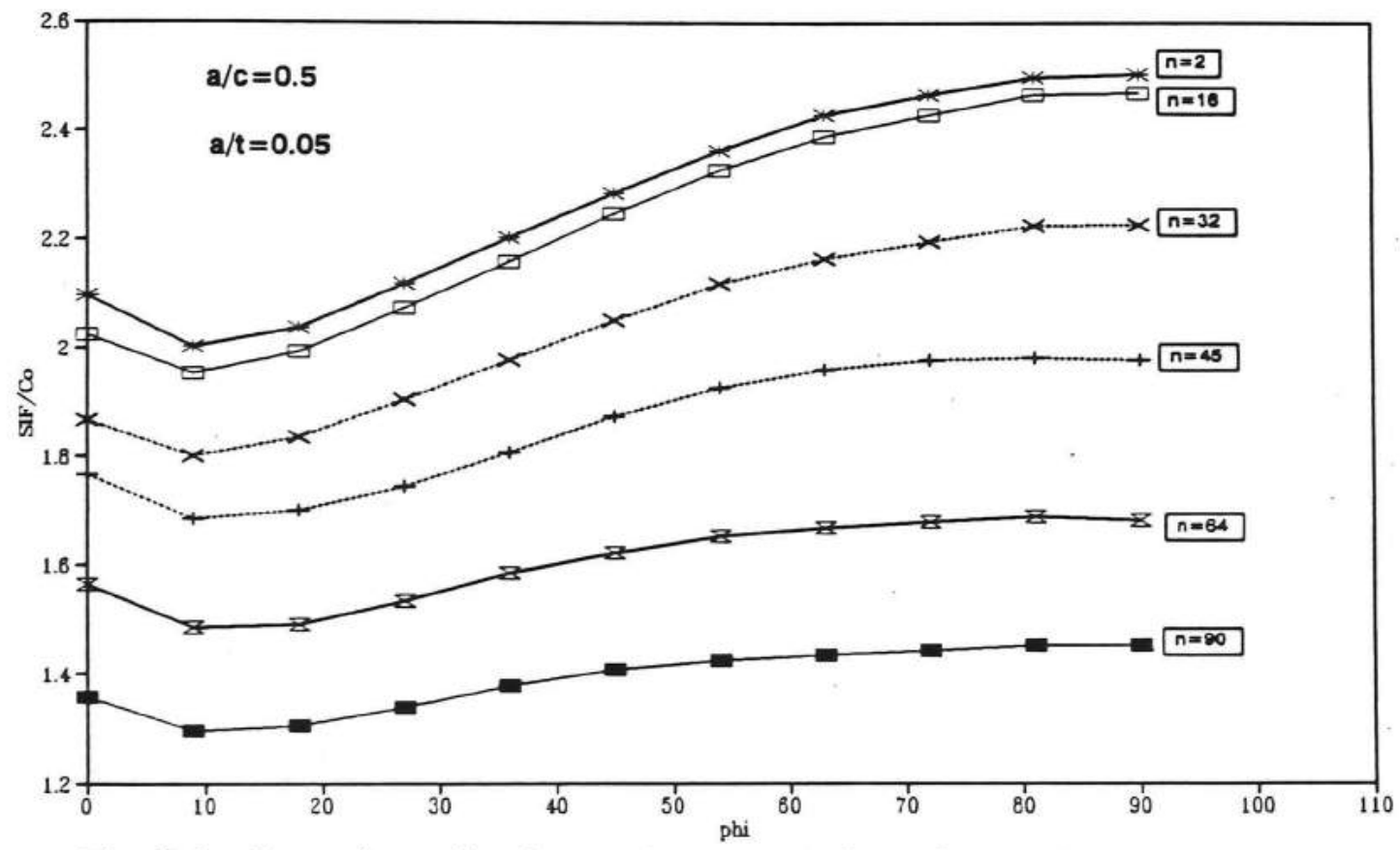

Fig.5.6 Stress intensity factor along crack-front for semi-elliptical surface crack in pressurized cylindrical vessel $(a / t=0.05)$. 


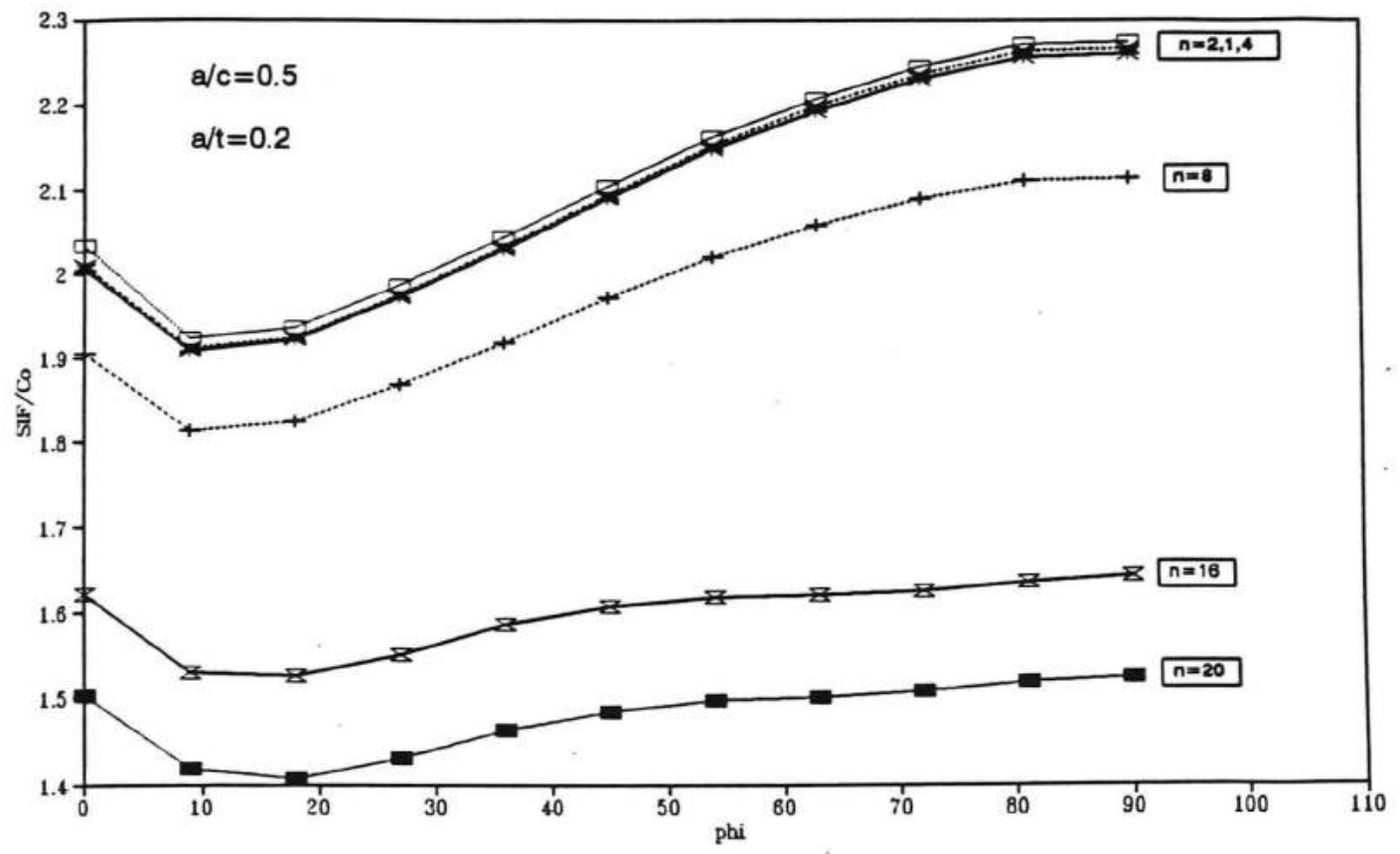

Fig.5.7 Stress intensity factor along crack-front for semi-elliptical surface crack in pressurized cylindrical vessel $(a / c=0.5, a / t=0.2)$.

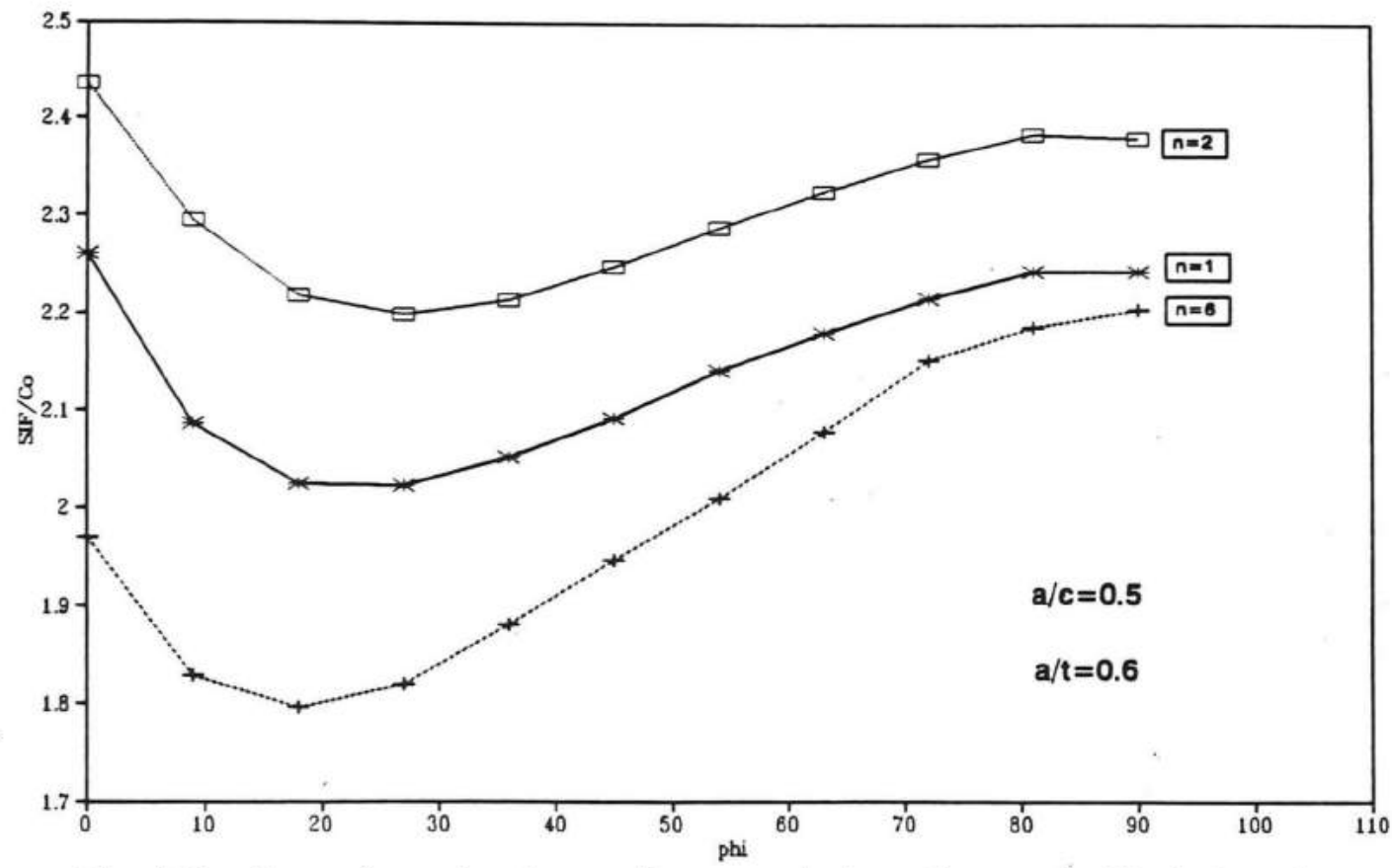

Fig.5.8 Stress intensity factor along crack-front for semi-elliptical surface crack in pressurized cylindrical vessel $(a / c=0.5, a / t=0.6)$. 
maximum SIF $\left(\phi=90^{\circ}\right)$ and the value of the SIF at $\phi=0^{\circ}$ decreases. For larger $a / t$, this phenomena occurs for smaller number of cracks.

- For $a / t=0.6$ (Fig.5.8), the maximum stress intensity factor shifts from $0^{\circ}$ ( $n=2$ cracks) to $90^{\circ}$ ( $n=6$ cracks). Therefore, it can be assumed that between $n=2$ and $n=6$ a transition occurs.

Figure 5.9 shows the variation of the normalized SIF for two semielliptical cracks $(a / c=0.5)$ as a function of the ratio crack depth to wall thickness, $a / t$, and the angle $\phi$.

- At the deepest point, $\phi=90^{\circ}$, the SIF decreases for a/t from 0.05 to 0.4 , but increases for $a / t=0.6$. At $\phi=0^{\circ}$, the SIF decreases for $a / t$ from 0.05 to 0.1 , but increases for $a / t$ from 0.2 to 0.6 .

Figure 5.10 shows the variation of the maximum $\operatorname{SIF}\left(\phi=90^{\circ}\right)$ for semi-elliptical cracks $(a / c=0.5)$ as a function of the number of cracks and the crack depth $(a / t)$.

For small $a / t$ the maximum SIF for $n=1$ to 16 is approximately equal, the difference between the SIFs becomes larger as the ratio a/t and the number of cracks, $n$, increases. 


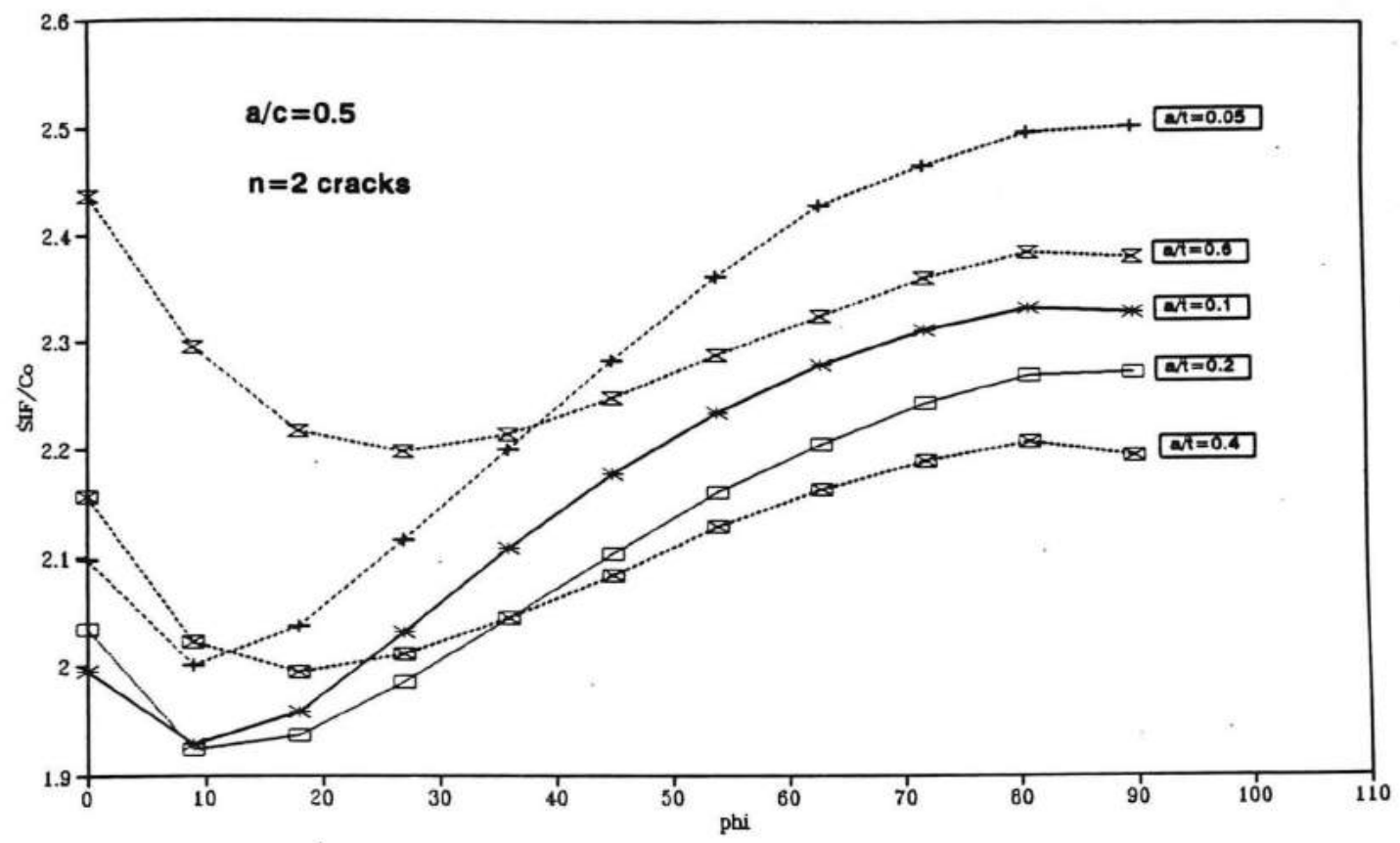

Fig.5.9 Stress intensity factor along crack-front for two semi-elliptical surface crack in pressurized cylindrical vessel $(a / c=0.5)$.

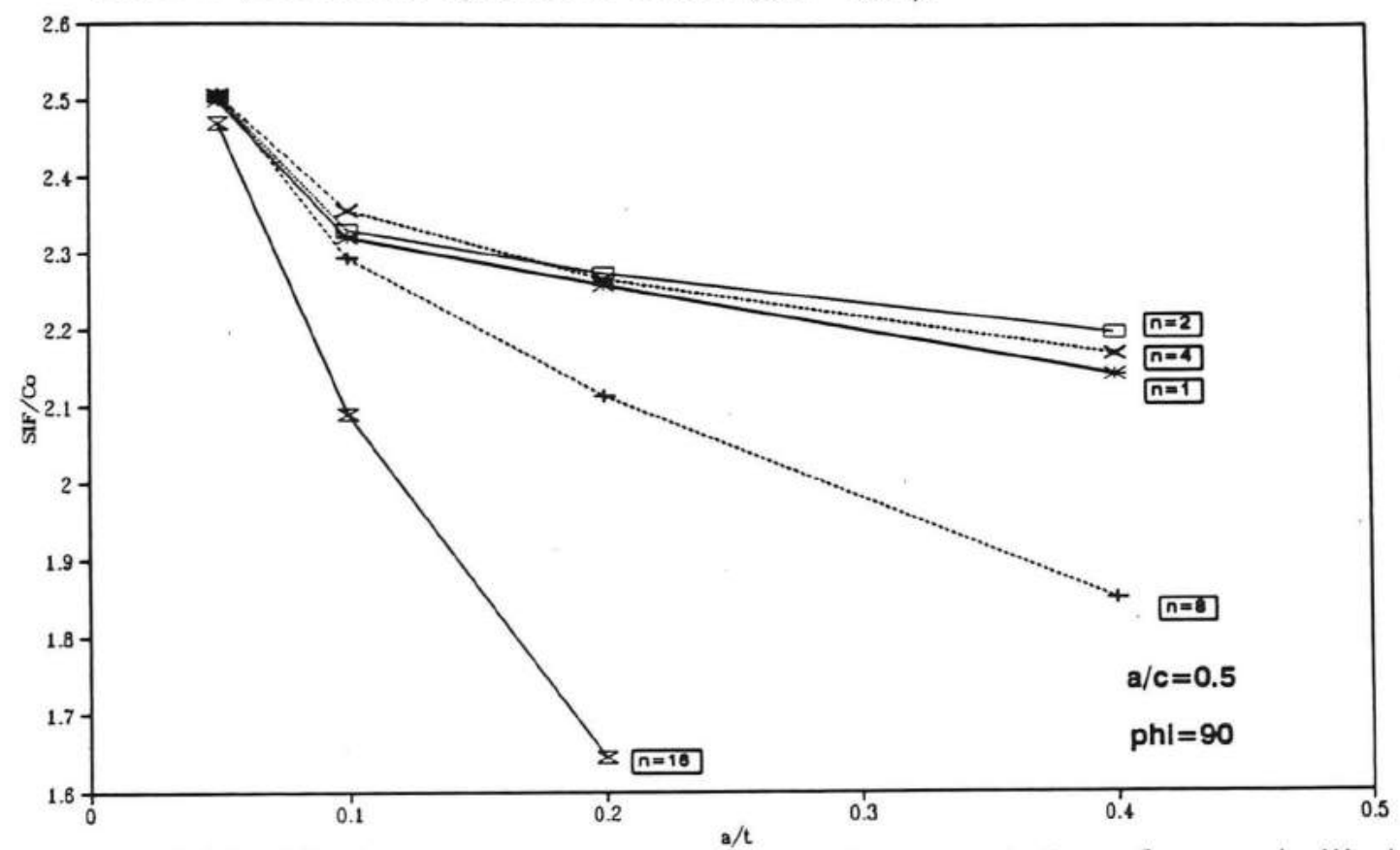

Fig.5.10 Maximum stress intensity factor along crack-front for semi-elliptical surface crack in pressurized cylindrical vessel $(a / c=0.5)$. 
Figures 5.11-5.13 show the variation of the SIF for semi-elliptical surface cracks $(a / c=0.2)$ and the ratio $a / t=0.05,0.1$, and 0.2 as a function of the angle, $\phi$, and the number of cracks, $n$.

- The maximum SIF occurs at the deepest point of the crack $\left(\phi=90^{\circ}\right)$ and the minimum at $\phi=9^{\circ}$.

- The SIF increases more rapidly from $\phi=9^{\circ}$ to $90^{\circ}$ than the $a / c=0.5$ case. For two shallow cracks, the difference between the maximum and the minimum SIF is $47 \%$ for $\mathrm{a} / \mathrm{c}=0.2$ and $a / t=0.05$ (Fig.5.11) versus $20 \%$ for $a / c=0.5$ and the same $a / t$ (Fig.5.6).

- The larger the ratio of crack depth to wall thickness, a/t, the larger the difference of the stress intensity factors between the $n=2$ and the $n=1$ cases. 


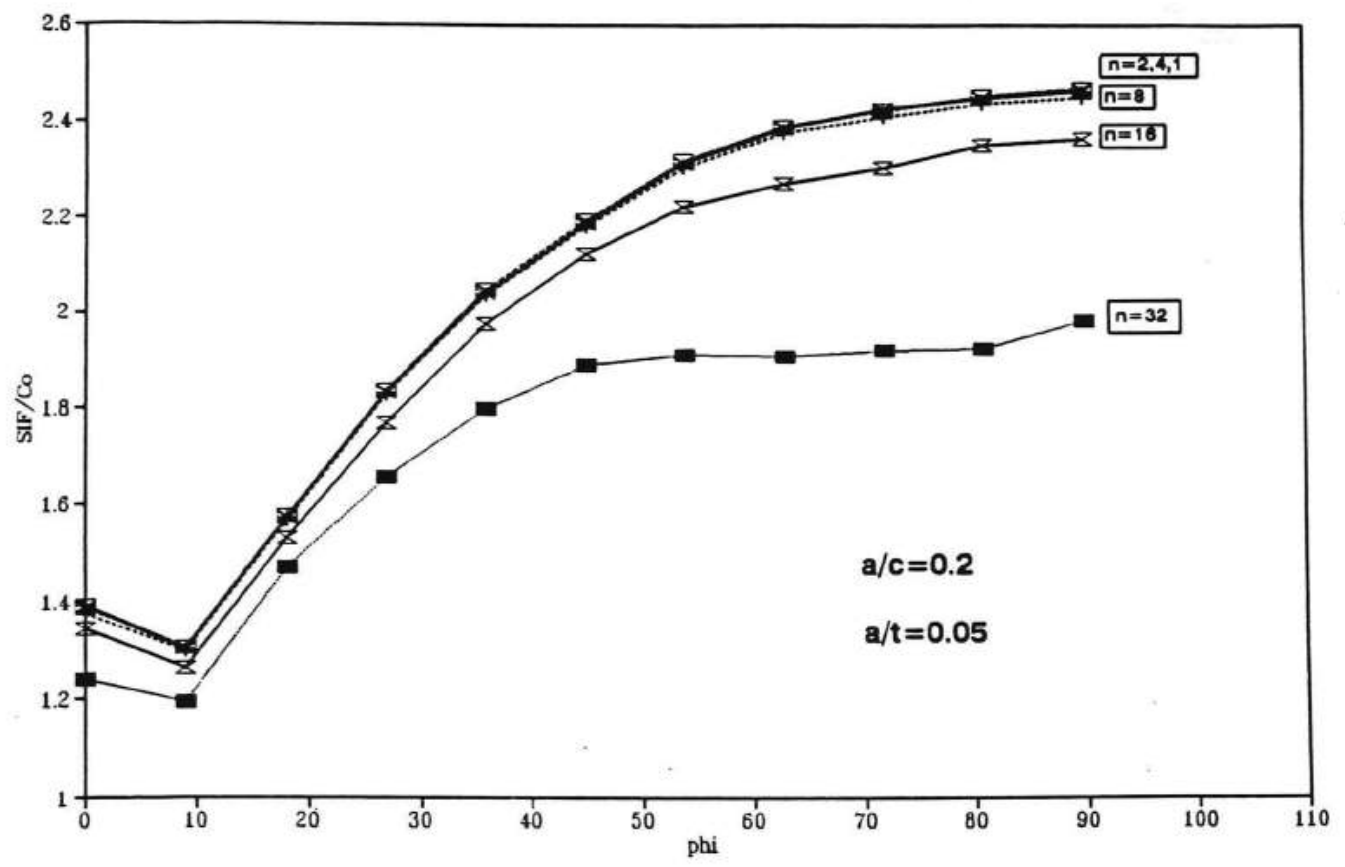

Fig.5.11 Stress intensity factor along crack-front for semi-elliptical surface crack in pressurized cylindrical vessel $(a / c=0.2, a / t=0.05)$

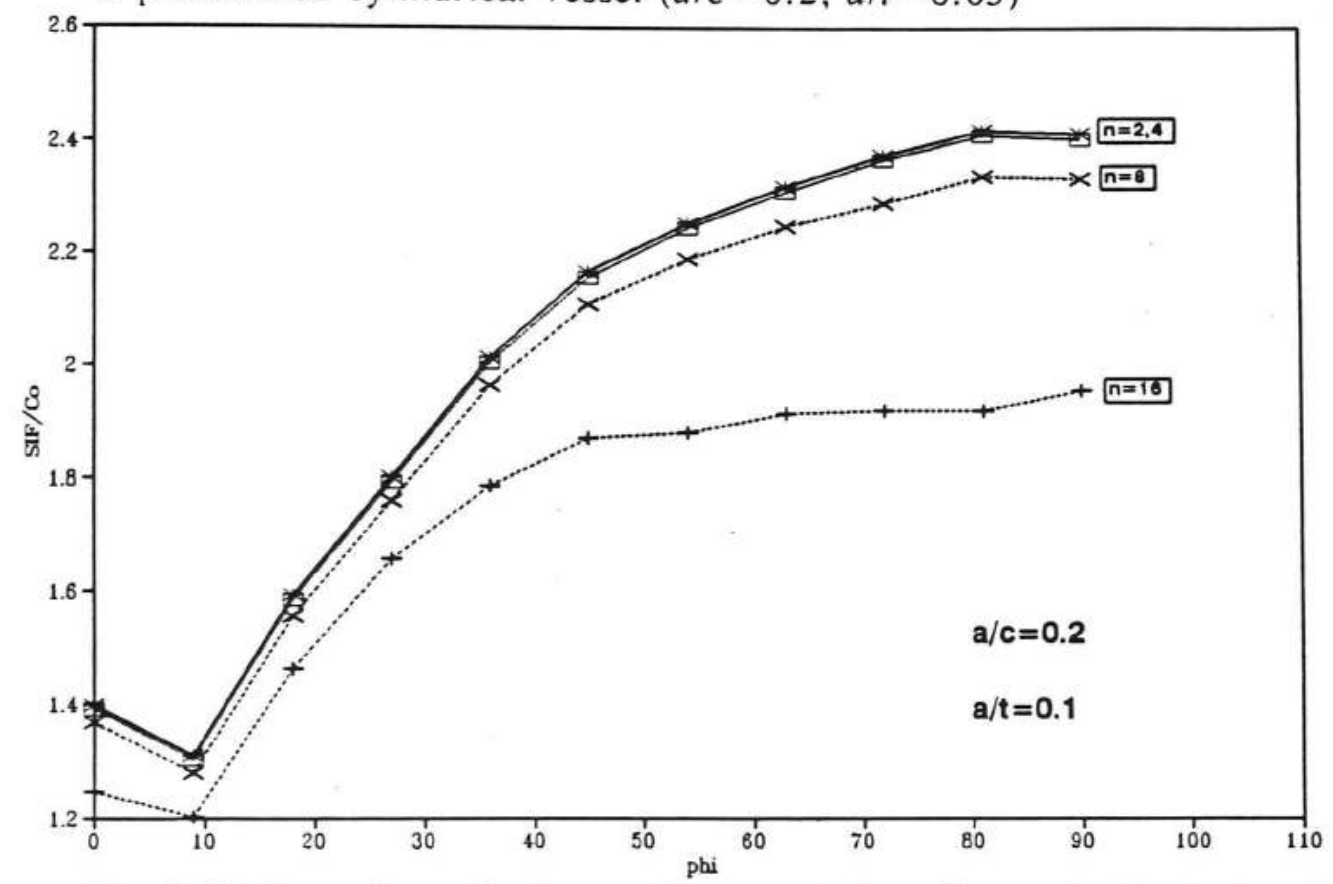

Fig.5.12 Stress intensity factor along crack-front for semi-elliptical surface crack in pressurized cylindrical vessel $(a / c=0.2, a / t=0.1)$. 


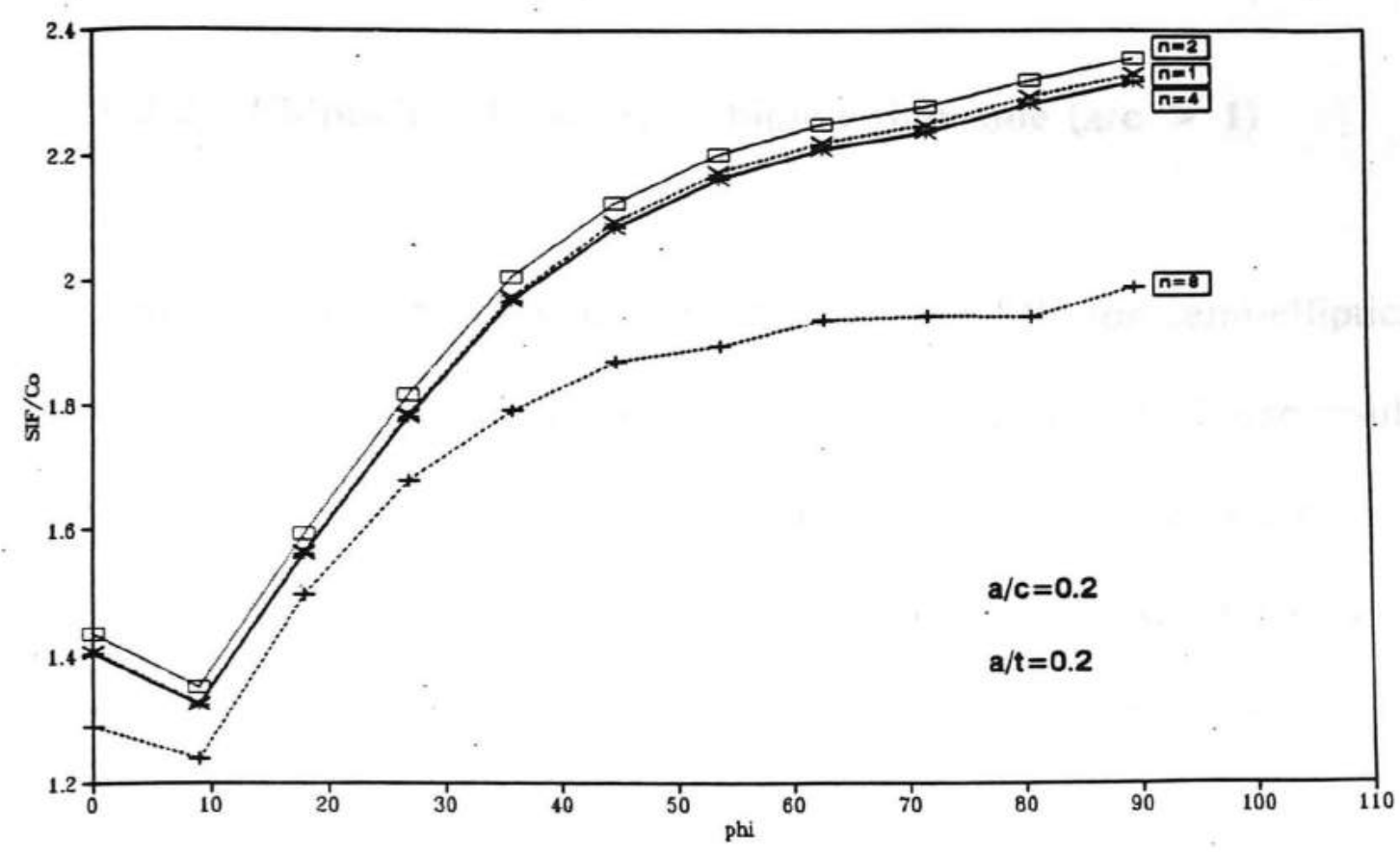

Fig.5.13 Stress intensity factor along crack-front for semi-elliptical surface crack in pressurized cylindrical vessel $(a / c=0.2, a / t=0.2)$

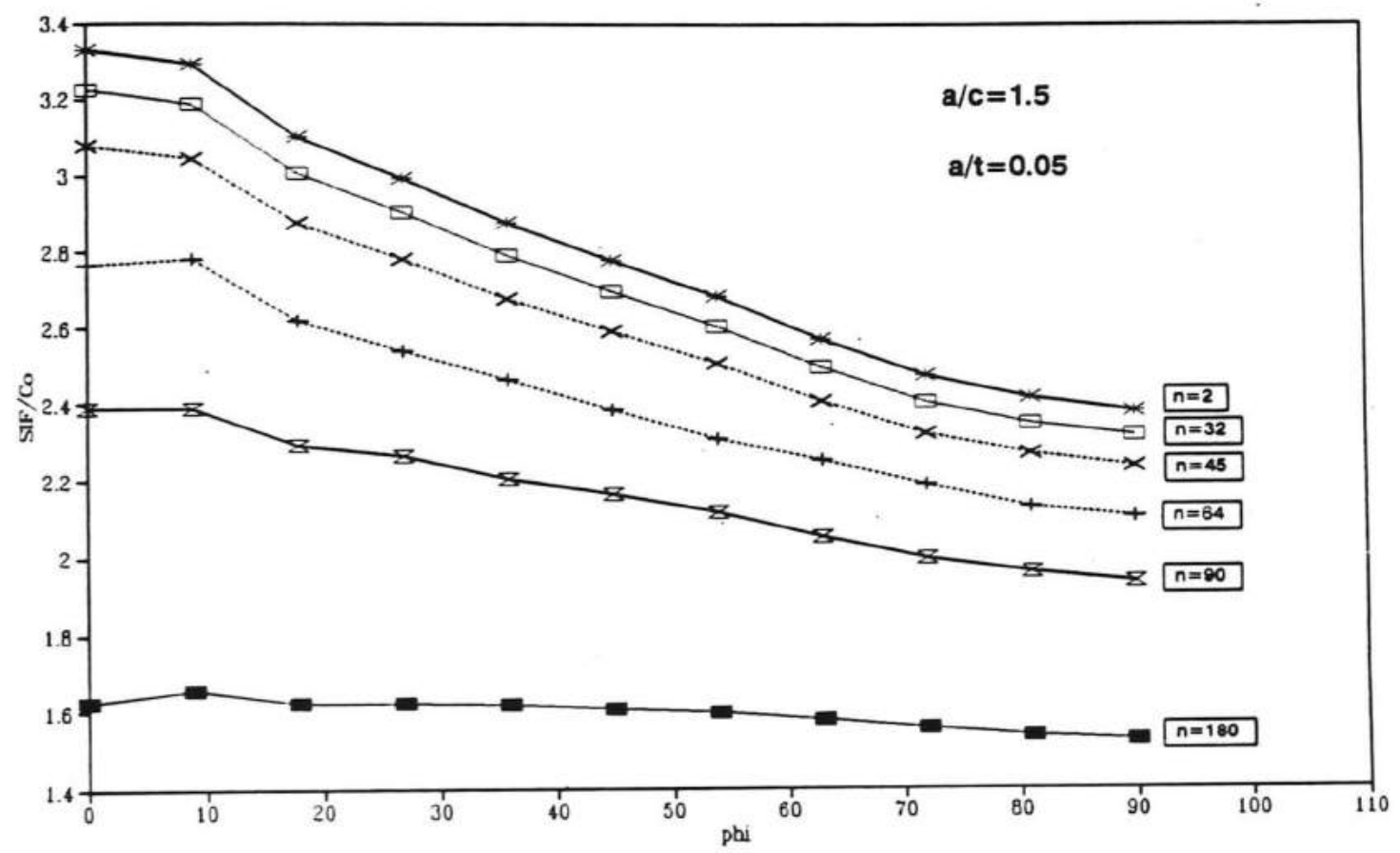

Fig.5.14 Stress intensity factor along crack-front for semi-elliptical surface crach in pressurized cylindrical vessel $(a / c=1.5, a / t=0.05)$. 


\subsubsection{Ellipticity of the crack higher than one $(\mathrm{a} / \mathrm{c}>1)$}

Figure 5.14-5.16 show the variation of the SIF for semi-elliptical surface cracks $(a / c=1.5)$ and the ratio $a / t=0.05,0.2$, and 0.6 . These results are given as a function of the angle, $\phi$, and the number of cracks, $n$.

- The shape of the curves is very similar to the case of the semicircular cracks. Even though the behavior of the SIF is the same for the semi-elliptical case, the SIF decreases more rapidly .

- For small number of cracks the maximum SIF occurs at $0^{\circ}$. As the number of cracks increases, the maximum stress intensity factor shifts from $\phi=0^{\circ}$ to approximately $9^{\circ}$.

Figure 5.17 shows the variation of the normalized SIF for two semielliptical cracks $(a / c=1.5)$ as a function of crack depth to wall thickness ratio, $a / t$, and the angle $\phi$.

- The normalized SIF decreases as the crack depth increases. 


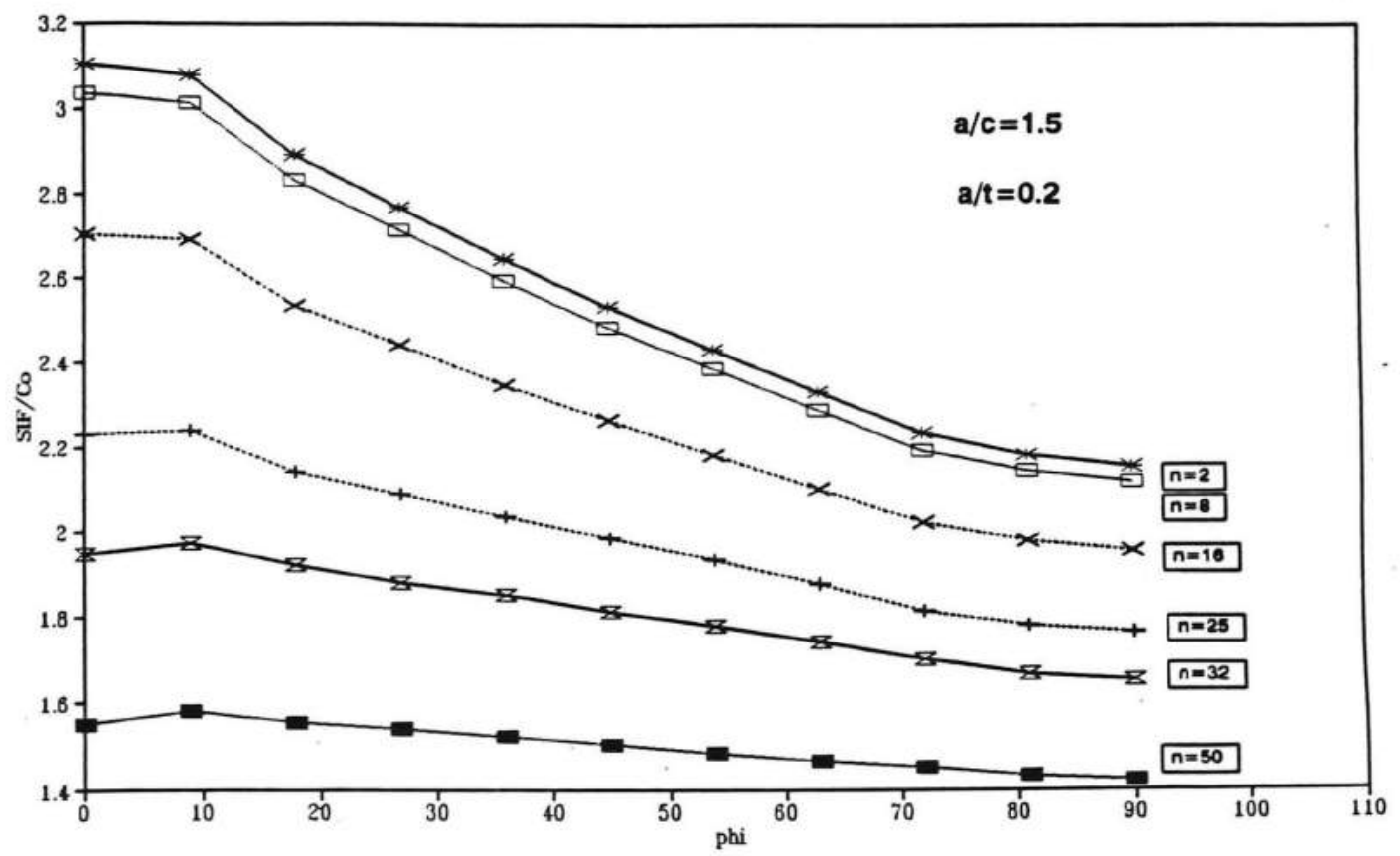

Fig.5.15 Stress intensity factor along crack-front for semi-elliptical surface crack in pressurized cylindrical vessel $(a / c=1.5, a / t=0.2)$

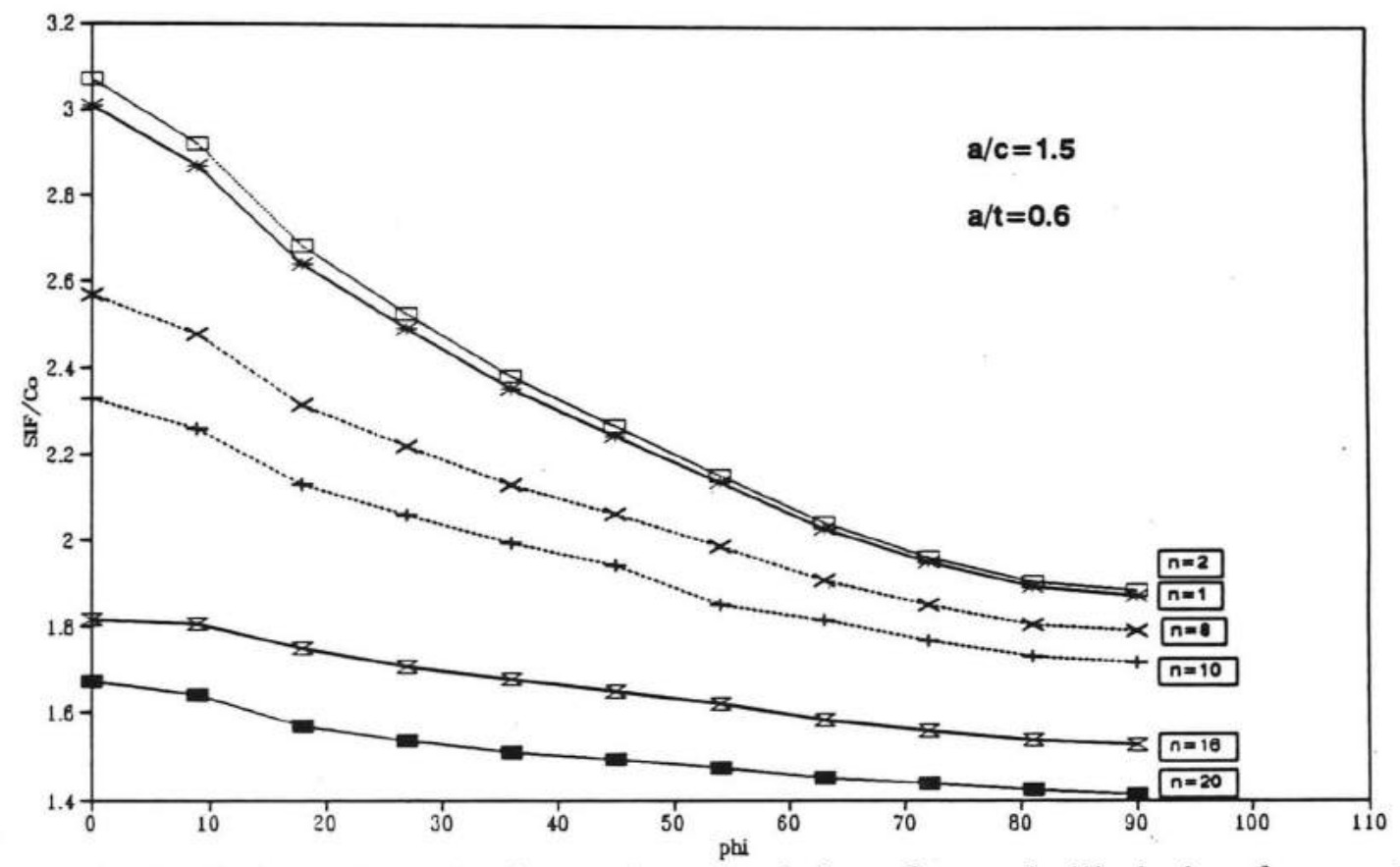

Fig.5.16 Stress intensity factor along crack-front for semi-elliptical surface crack in pressurized cylindrical vessel $(a / c=1.5, a / t=0.6)$. 


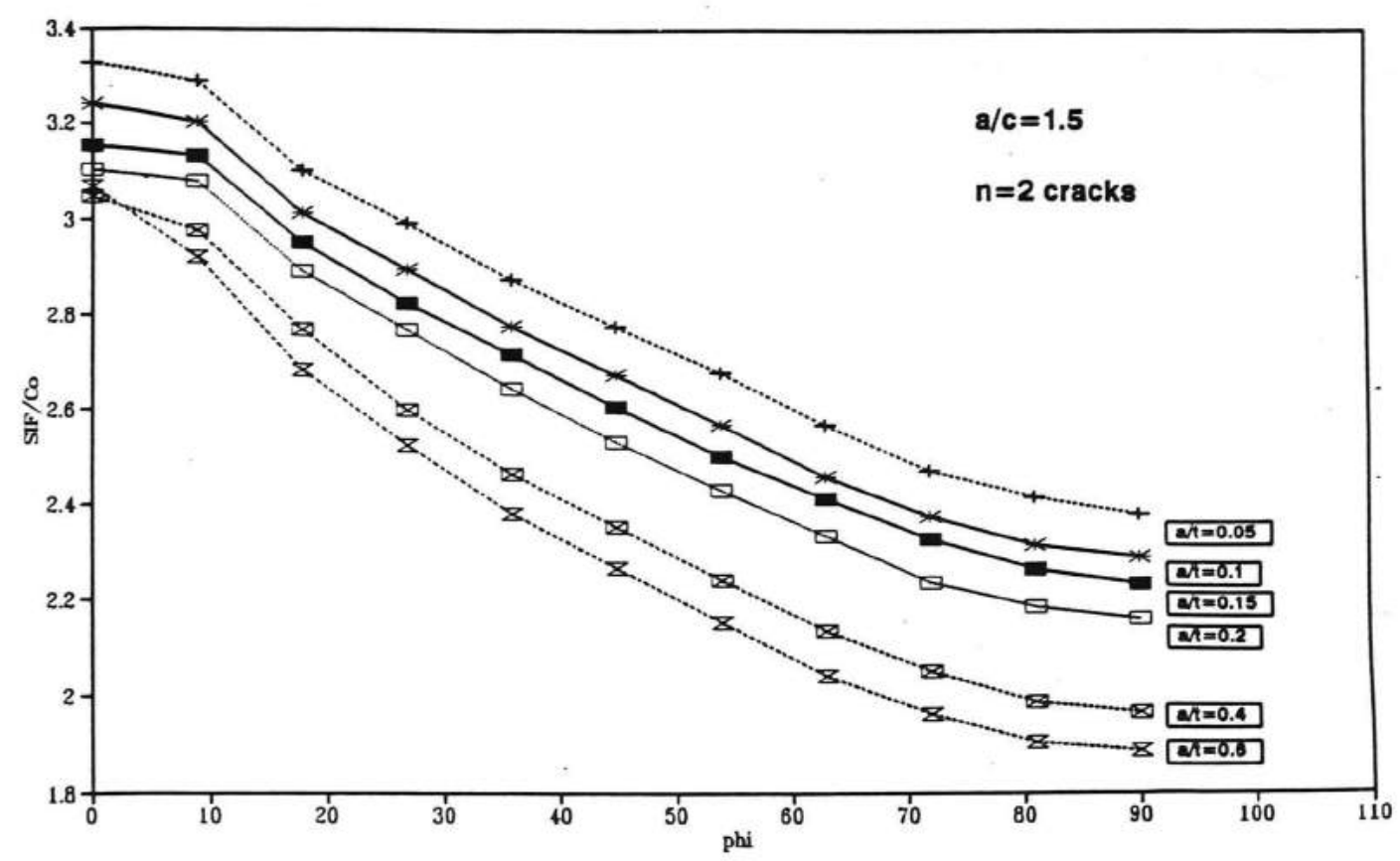

Fig.5.17 Stress intensity factor along crack-front for two semi-elliptical surface crack in pressurized cylindrical vessel $(a / c=1.5)$

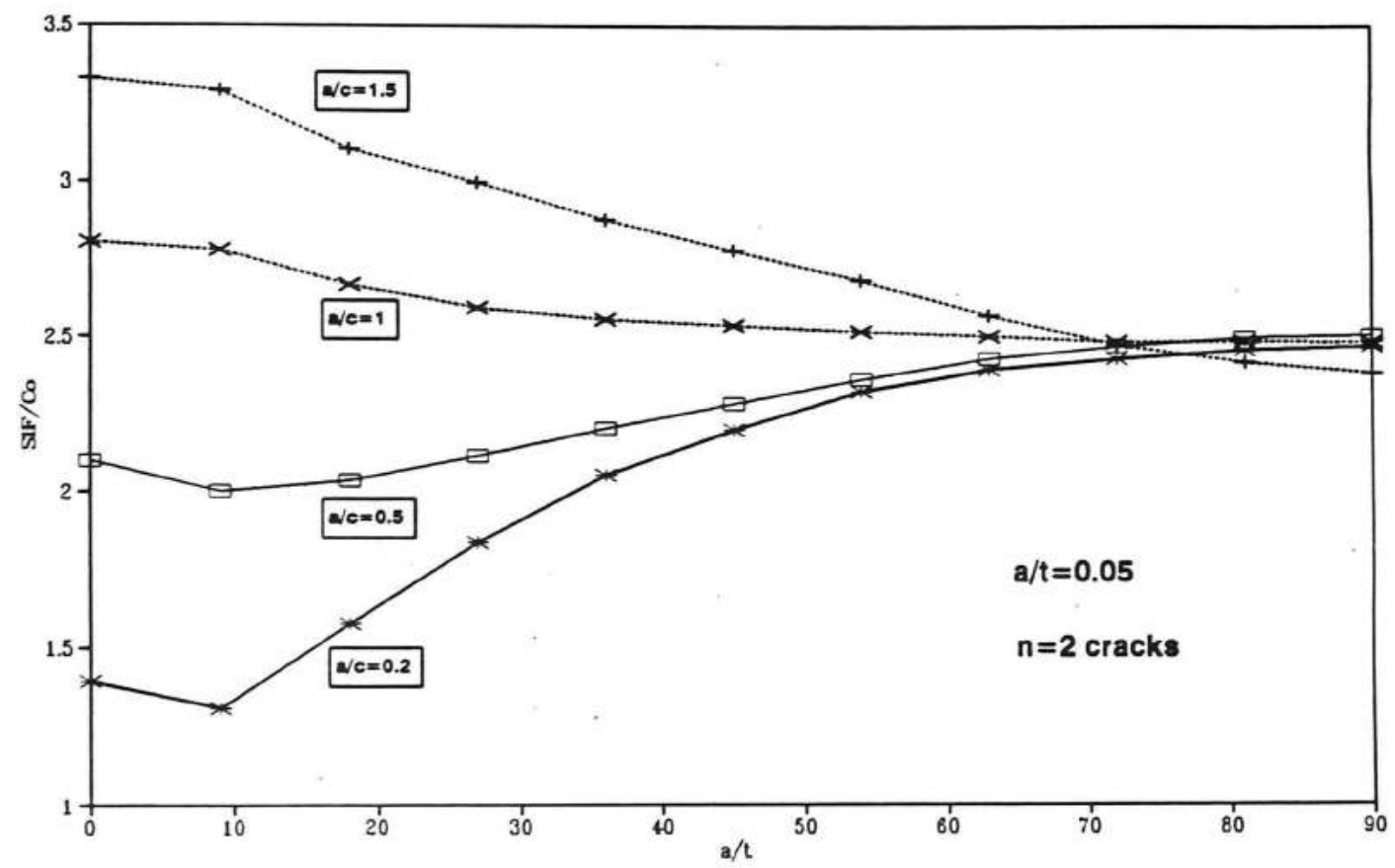

Fig.5.18 Stress intensity factor along crack-front for two surface cracks $(a / t=0.05)$ of different ellipticity as a function of the crack depth. 
Figures 5.18 and 5.19 show the normalized SIF for two cracks and $a / t=0.05$ and 0.2 respectively, as a function of the number of cracks, $n$, and the ellipticity of the crack, $a / c$. For the smallest crack, $a / t=0.05$ (Fig.5.18), from $0^{\circ}$ to $70^{\circ}$ the smaller the ellipticity the smaller the normalized SIF, from $70^{\circ}$ to $90^{\circ}$ the normalized SIF tends to a constant value, 2.5 , except for $a / c=1.5$, where the normalized SIF is 2.38 .

For $a / t=0.2$ (Fig.5.19), the behavior is very similar. The smaller the value of $a / c$ the smaller the SIF, but the angle of transition is smaller, $\phi=40^{\circ}$. At the deepest point of the crack $\left(\phi=90^{\circ}\right)$ the SIF is higher for smaller values of $a / c$.

In order to show the variation of the SIF with the ratio of the thickness of the cylinder to inner radius, $t / r_{i}$, some cases with different $t / r_{i}$ were solved. Figure 5.20 shows the variation of the SIF as a function of $t / r_{i}$, for $a / c=0.5, a / t=0.4$ and for $a / c=1, a / t=0.2$. As the ratio $t / r_{i}$ decreases the stress intensity factor increases as expected. Thus, thinner cylinders exhibit more dangerous cracks than thicker cylinder for the same $a / c$ ratio. 


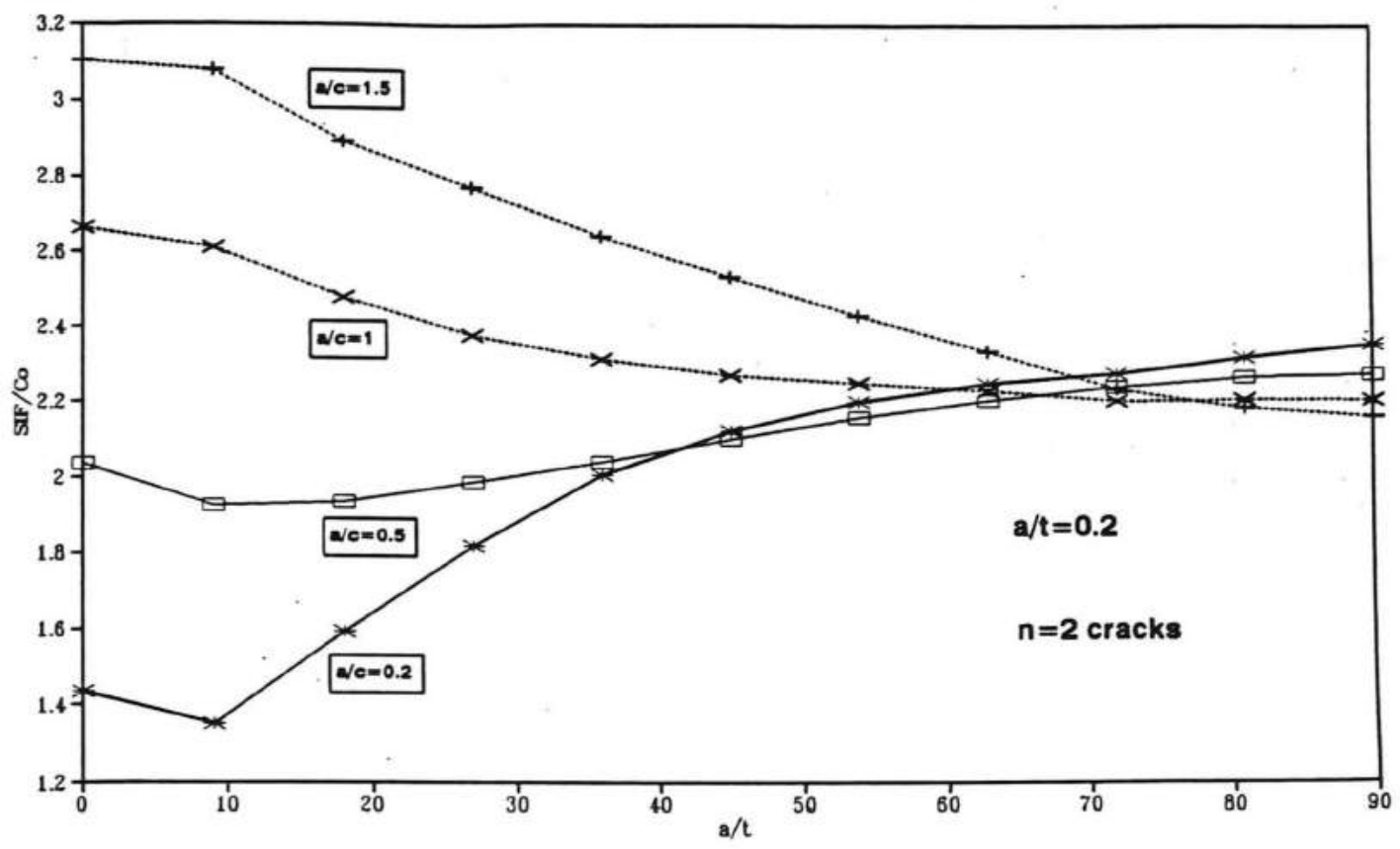

Fig.5.19 Stress intensity factor along crack-front for two surface cracks $(a / t=0.2)$ of different ellipticity as a function of the crack depth.

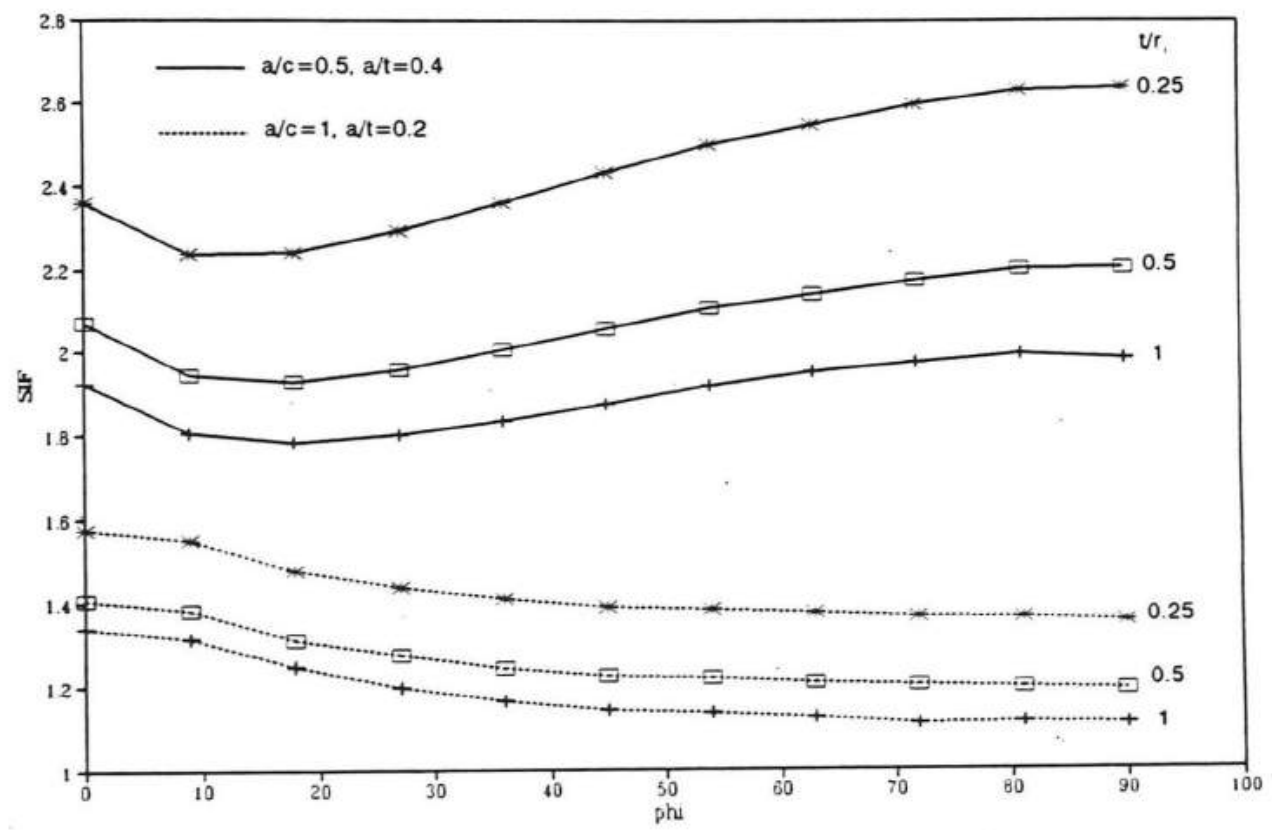

Fig.5.20 Variation of the stress intensity factor along crack-front as a function of $t / r_{i}$. 


\subsection{Conclusions}

The stress intensity factor was calculated using a three-dimensional finite element analysis for different radial configurations of semi-circular and semi-elliptical internal surface cracks in a pressurized thick walled cylinder. The finite element models were meshed with ten-node tetrahedral elements and had between 7500 to 10000 degrees of freedom. A technique of substructuring was used, building a submodel with twenty-node cubic isoparametric elements and singular elements along the crack front. The nodal displacement method was used to calculate the stress intensity factor.

In order to validate these results, two different cases were solved using this method and compared to other analyses of internal surface radial cracks in cylinders. The present results were in very good agreement with other finite element analyses.

The stress intensity factors for different crack configurations were presented. The ratio of crack depth to crack length ranged from 0.2 to 1.5 , the ratio of crack depth to wall thickness ranged from 0.05 to 0.6 and the 
number of cracks from $n=1$ to 180 where possible.

The stress intensity factors for all the cases solved in this study were maximum for two equispaced cracks. For semi-circular surface cracks the stress intensity factors were maximum at the intersection of the crack with the inner surface of the cylinder and minimum at the deepest point of the crack. As for semi-elliptical surface cracks, when $a / c$ was less than 1 the maximum SIFs were at the deepest point of the crack, except for small number of large cracks $(n=2, a / t=0.6)$ where the maximum SIF shifted from the deepest point to the intersection with the inner surface, $\phi=0^{\circ}$. When $a / c$ was higher than 1 the maximum SIFs were at the intersection of the crack with the inside surface, but as the number of cracks increased the maximum occured at an interior point. The smaller the crack the smaller the difference between the two cracks case and the single crack case. The larger the number of cracks the smaller the variation of the SIFs with the angle $\phi$. As the thickness of the cylinder decreased, the SIFs were found increase.

An empirical equation to estimate the maximum SIF for radial of internal semi-circular and semi-elliptical surface cracks in pressurized thick walled cylinders was developed in this study. The values obtained by 
equation (3.26) were within $5.7 \%$ of the finite element results. This empirical equation is valid for $t / r_{i}$ close to 1 ; a comparison of the results obtained by this equation and those obtained by Raju and Newman [12] was not possible because results in [12] were obtained for $t / r_{i}$ less or equal than 0.25 . Also, we were unable to compare the results of the empirical equation with the limiting case of infinite wall thickness because the limited wave front in our version of ANSYS.

The stress intensity factors obtained in this study should provide a more realistic estimation of the static fracture endurance, the crack-growth rate and the total fatigue life of multicracked thick walled pressurized cylinders, since all the empirical formulas dealing with these subjects are function of $K_{I}$.

\subsection{Future research}

In the present study, the stress intensity factor for radial surface cracks in pressurized cylinders were calculated using a three-dimensional finite element analysis (ANSYS). A substructuring technique was also 
introduced. This method provides a very useful tool which can be applied for a variety of three-dimensional linear fracture problems.

In particular, the method of substructuring has been applied to evaluate the three-dimensional SIF distribution along the crack front for coplanar arrays of internal semi-circular and semi-elliptical cracks in pressurized thick walled cylinders. As a future research, this method can be applied to analyze the combination of large arrays of radial and coplanar surface internal and external cracks. In the same manner, surface cracks near the end of the cylinder under pressure and different crack configurations of surface cracks in cylinders under internal thermal shock can be considered. 


\section{REFERENCES}

[1] Underwood, J. H., "Stress Intensity Factors for Internally Pressurized Thick-Walled Cylinders," Stress Analysis and Growth of Cracks, ASTM STP-513, American Society for Testing and Materials, pp. 59-70 (1972).

[2] Kobayashi, A. S., "A Simple Procedure for Estimating Stress Intensity Factors in Regions of High Stress Gradient," Significance of Defects in Welded Structures, eds., T. Kanazawa and A. S. Kobayashi, University of Tokyo Press, pp. 127-143 (1974).

[3] Kobayashi, A. S., Emery, A. F., Polvanich, N., and Love, W. J., "Inner and Outer Surface Cracks in Internally Pressurized Cylinders," ASME Journal of Pressure Vessel Technology, pp. 83-89 (1977).

[4] Tracy, P. G., "Elastic Analysis of Radial Cracks Emanating from the Outer and Inner Surfaces of a Circular Ring," Engineering Fracture Mechanics, 11, pp. 291-300 (1979).

[5] Baratta, F. I., "Stress Intensity Factors for Internal Multiple Cracks in Thick-Walled Cylinders Stressed by Internal Pressure Using Load Relief Factors," Engineering Fracture Mechanics, 10, pp. 691-697 (1979).

[6] Pu, S. L. and Houssain, M. A., "Stress Intensity Factors for a 
Circular Ring with a Uniform Array of Radial Cracks Using Isoparametric Singular Elements,"Fracture Mechanics, ASTM, STP 677, pp. 685-699 (1979).

[7] Perl, M. and Aroné, R., "Stress Intensity Factors for Large Arrays of Radial Cracks in Thick-Walled Steel Cylinders, "Engineering Fracture Mechanics, 25, pp. 341-348 (1986).

[8] Perl, M. and Aroné, R., "Stress Intensity Factors for a Radially Multicracked Partially Autofrettaged Pressurized Thick-Walled Cylinder," Trans. of the ASME, J. of Pressure Vessel Tech., 110, pp. 147-154 (1988).

[9] Perl, M. and Ashkenazi, A., "Radial Cracking of a Thick-Walled Cylinder Due to an Internal Thermal Shock," accepted for publication by Engineering Fracture Mechanics.

[10] Atluri, S. N., and Kathiresan, K., "Outer and Inner Surface Flaws in Thick-Walled Pressure Vessels," Transactions of the Fourth International Conference on Structural Mechanics in Reactor Technology, San Francisco, Calif. (1977).

[11] McGowan, J. J., and Raymund, M., "Stress Intensity Factors SolutionsFor Internal Longitudinal Semi-Elliptical Surface Flaws in a Cylinder under Arbitrary Loadings, "Fracture Mechanics, ASTM STP-677, American Society for Testing and Materials, pp. 365-380 (1979). 
[12] Raju, I. S. and Newman Jr., J. C., "Stress Intensity Factors for Internal and External Surface Cracks in Cylindrical Vessels, " Trans. ASME, J. of Pressure Vessel Tech., 104, pp. 293-298 (1982).

[13] Kirkhope, K. J., Bell, R. and Kirkhope, J., "Evaluation of 3-D Stress Intensity Factors in Multicrackes Thick-Walled Cylinder," Numerical Methods in Fracture Mechanics, A. R. Luxmoore and D. R. J. Owen, Eds., Pineridge Press, pp. 179-193 (1984).

[14] Kirkhope, K. J., Bell, R. and Kirkhope, J., "Stress Intensity Factors for Single and Multiple Semi-Elliptical Surface Cracks in Pressurized ThickWalled Cylinders," International Journal of Pressure Vessels and Piping, pp. 247-257 (1991).

[15] O’Donoghue, P. E., Nishioka, T. and Atluri, S. N., "Multiple Surface Cracks in Pressure Vessels, "Engineering Fracture Mechanics, 20, pp. 545-560 (1984).

[16] O’Donoghue, P. E., Nishioka, T. and Atluri, S. N., "Analysis of Interaction Behavior of Surface Flaws in Pressure Vessels," Trans. ASME, J. of Pressure Vessel Tech.,108, pp. 24-32 (1986).

[17] Parker, E. R., Brittle Behavior of Engineering Structures, John Wiley, New York (1957).

[18] Kobayashi, A. S., "Experimental Techniques in Fracture Mechanics," 
Society for Experimental Stress Analysis, Westport, Conn., pp. 4-5 (1973). [19] Broek, D., "Elementary Engineering Fracture Mechanics," Kluwer Academic, Dordretch, The Netherlands, pp. 7-8 (1991).

[20] Irons, B. M., "A Frontal Solution Program for Finite Element Analysis," International Journal for Numerical Methods in Engineering, 2, pp. 5-23 (1970).

[21] Tong, P. and Piau, T. H. H., "On the Convergence of the Finite Element Methods for Problems with Singularity," International Journal of Solids and Structures, 9, pp. 313-321 (1973).

[22] Barsoum, R. S., "On the Use of Isoparametric Finite Elements in Linear Fracture Mechanics, "International Journal for Numerical Methods in Engineering, 10, pp. 25-37 (1976).

[23] Chan, S. K., Tuba, I. S. and Wilson, W. K., "On the Finite Element Methods in Linear Fracture Mechanics," Engineering Fracture Mechanics, 2, pp. 4-9 (1970).

[24] Paris, P. C. and Sih, G. C., "Stress Analysis of Cracks in Fracture Toughness and Testing and its Applications," American Society for Testing and Materials, Philadelpia, STP 381, pp. 30-83 (1965).

[25] Shiva Kumar, K. N., Tan, P. W. and Newman Jr., J. C., International Journal of Fracture, 20, pp. 43-50 (1988). 
[26] Solecki, J. S.,"Fracture Mechanics Tutorial," Swanson Analysis Systems, Inc., Houston, Pa. (1989). 


\section{APPENDIX A}

TABLES OF NORMALIZED STRESS INTENSITY FACTORS 
Table A1. Normalized SIF for various number of cracks $(a / c=0.2, a / t=0.05)$

\begin{tabular}{|c|c|c|c|c|c|c|c|c|}
\hline $\mathrm{a} / \mathrm{c}$ & $\mathrm{a} / \mathrm{t}$ & $\mathrm{phi}$ & 1 & 2 & 4 & 8 & 16 & 32 \\
\hline & & & & & & & & \\
\hline 0.2 & 0.05 & 0 & 1.3903 & 1.3924 & 1.3874 & 1.3748 & 1.3446 & 1.2390 \\
\hline 0.2 & 0.05 & 9 & 1.3031 & 1.3063 & 1.3046 & 1.2981 & 1.2624 & 1.1936 \\
\hline 0.2 & 0.05 & 18 & 1.5741 & 1.5763 & 1.5742 & 1.5664 & 1.5321 & 1.4693 \\
\hline 0.2 & 0.05 & 27 & 1.8337 & 1.8388 & 1.8362 & 1.8271 & 1.7701 & 1.6578 \\
\hline 0.2 & 0.05 & 36 & 2.0418 & 2.0505 & 2.0478 & 2.0375 & 1.9787 & 1.8012 \\
\hline 0.2 & 0.05 & 45 & 2.1896 & 2.1977 & 2.1948 & 2.1837 & 2.1271 & 1.8901 \\
\hline 0.2 & 0.05 & 54 & 2.3179 & 2.3213 & 2.3184 & 2.3064 & 2.2270 & 1.9150 \\
\hline 0.2 & 0.05 & 63 & 2.3888 & 2.3928 & 2.3900 & 2.3777 & 2.2734 & 1.9126 \\
\hline 0.2 & 0.05 & 72 & 2.4289 & 2.4275 & 2.4248 & 2.4117 & 2.3073 & 1.9238 \\
\hline 0.2 & 0.05 & 81 & 2.4489 & 2.4542 & 2.4517 & 2.4383 & 2.3533 & 1.9266 \\
\hline 0.2 & 0.05 & 90 & 2.4632 & 2.4688 & 2.4663 & 2.4527 & 2.3645 & 1.9853 \\
\hline
\end{tabular}

Table A2. Normalized SIF for various number of cracks $(a / c=0.2, a / r=0.1)$

n

\begin{tabular}{|c|c|c|c|c|c|c|}
\hline $\mathrm{a} / \mathrm{c}$ & $\mathrm{a} / \mathrm{t}$ & $\mathrm{phi}$ & 2 & 4 & 8 & 16 \\
\hline & & & & & & \\
\hline 0.2 & 0.10 & 0 & 1.3993 & 1.3924 & 1.3672 & 1.2461 \\
\hline 0.2 & 0.10 & 9 & 1.3088 & 1.3024 & 1.2789 & 1.2013 \\
\hline 0.2 & 0.10 & 18 & 1.5928 & 1.5851 & 1.5558 & 1.4626 \\
\hline 0.2 & 0.10 & 27 & 1.8028 & 1.7945 & 1.7598 & 1.6590 \\
\hline 0.2 & 0.10 & 36 & 2.0134 & 2.0046 & 1.9633 & 1.7858 \\
\hline 0.2 & 0.10 & 45 & 2.1654 & 2.1562 & 2.1085 & 1.8703 \\
\hline 0.2 & 0.10 & 54 & 2.2521 & 2.2429 & 2.1888 & 1.8804 \\
\hline 0.2 & 0.10 & 63 & 2.3163 & 2.3073 & 2.2457 & 1.9140 \\
\hline 0.2 & 0.10 & 72 & 2.3757 & 2.3668 & 2.2884 & 1.9196 \\
\hline 0.2 & 0.10 & 81 & 2.4181 & 2.4093 & 2.3381 & 1.9193 \\
\hline 0.2 & 0.10 & 90 & 2.4132 & 2.4045 & 2.3324 & 1.9554 \\
\hline
\end{tabular}


Table A3. Normalized SIF for various number of cracks $(a / c=0.2, a / t=0.15)$

\begin{tabular}{|c|c|c|c|c|c|c|}
\hline \multicolumn{6}{|c|}{$\mathbf{n}$} \\
\hline & & & & 4 & 6 & 12 \\
\hline 0.2 & 0.15 & 0 & 1.4142 & 1.3864 & 1.3697 & 1.2640 \\
\hline 0.2 & 0.15 & 9 & 1.3212 & 1.3176 & 1.2911 & 1.2125 \\
\hline 0.2 & 0.15 & 18 & 1.5900 & 1.5733 & 1.5415 & 1.4718 \\
\hline 0.2 & 0.15 & 27 & 1.8026 & 1.7848 & 1.7476 & 1.6473 \\
\hline 0.2 & 0.15 & 36 & 1.9908 & 1.9786 & 1.9353 & 1.7595 \\
\hline 0.2 & 0.15 & 45 & 2.1306 & 2.1123 & 2.0634 & 1.8325 \\
\hline 0.2 & 0.15 & 54 & 2.2156 & 2.1978 & 2.1443 & 1.8488 \\
\hline 0.2 & 0.15 & 63 & 2.2667 & 2.2497 & 2.1927 & 1.8408 \\
\hline 0.2 & 0.15 & 72 & 2.2962 & 2.2799 & 2.2191 & 1.8494 \\
\hline 0.2 & 0.15 & 81 & 2.3407 & 2.3246 & 2.2600 & 1.8408 \\
\hline 0.2 & 0.15 & 90 & 2.3729 & 2.3568 & 2.2896 & 1.8895 \\
\hline
\end{tabular}

Table A4. Normalized SIF for various number of cracks $(a / c=0.2, a / t=0.2)$

\begin{tabular}{|c|c|c|c|c|c|c|}
\hline$a / c$ & $a / t$ & phi & 1 & 2 & 4 & 8 \\
\hline 0.2 & 0.20 & 0 & 1.4037 & 1.4347 & 1.4056 & 1.2888 \\
\hline 0.2 & 0.20 & 9 & 1.3225 & 1.3509 & 1.3244 & 1.2364 \\
\hline 0.2 & 0.20 & 18 & 1.5628 & 1.5953 & 1.5655 & 1.4979 \\
\hline 0.2 & 0.20 & 27 & 1.7828 & 1.8187 & 1.7872 & 1.6804 \\
\hline 0.2 & 0.20 & 36 & 1.9704 & 2.0088 & 1.9768 & 1.7935 \\
\hline 0.2 & 0.20 & 45 & 2.0877 & 2.1273 & 2.0963 & 1.8746 \\
\hline 0.2 & 0.20 & 54 & 2.1633 & 2.2030 & 2.1738 & 1.8997 \\
\hline 0.2 & 0.20 & 63 & 2.2108 & 2.2509 & 2.2217 & 1.9404 \\
\hline 0.2 & 0.20 & 72 & 2.2378 & 2.2780 & 2.2497 & 1.9455 \\
\hline 0.2 & 0.20 & 81 & 2.2806 & 2.3197 & 2.2934 & 1.9438 \\
\hline 0.2 & 0.20 & 90 & 2.3160 & 2.3537 & 2.3288 & 1.9918 \\
\hline
\end{tabular}


Table A5. Normalized SIF for various number of $\operatorname{cracks}(a / c=0.5, a / t=0.05)$

\begin{tabular}{|c|c|c|c|c|c|c|c|c|c|c|c|}
\hline$a / c$ & $a / t$ & phi & 1 & 2 & 4 & 8 & 16 & 32 & 45 & 64 & 90 \\
\hline & & & & & . & & & & & & \\
\hline 0.5 & 0.05 & 0 & 2.0930 & 2.0983 & 2.0980 & 2.0481 & 2.0242 & 1.8662 & 1.7663 & 1.5652 & 1.3593 \\
\hline 0.5 & 0.05 & 9 & 1.9965 & 2.0016 & 2.0012 & 1.9758 & 1.9524 & 1.7994 & 1.6849 & 1.4850 & 1.2971 \\
\hline 0.5 & 0.05 & 18 & 2.0316 & 2.0367 & 2.0365 & 2.0178 & 1.9936 & 1.8353 & 1.7009 & 1.4915 & 1.3046 \\
\hline 0.5 & 0.05 & 27 & 2.1116 & 2.1169 & 2.1169 & 2.0979 & 2.0723 & 1.9039 & 1.7450 & 1.5338 & 1.3383 \\
\hline 0.5 & 0.05 & 36 & 2.1960 & 2.2014 & 2.2018 & 2.1858 & 2.1583 & 1.9772 & 1.8058 & 1.5849 & 1.3796 \\
\hline 0.5 & 0.05 & 45 & 2.2774 & 2.2830 & 2.2839 & 2.2758 & 2.2459 & 2.0502 & 1.8726 & 1.6238 & 1.4083 \\
\hline 0.5 & 0.05 & 54 & 2.3568 & 2.3626 & 2.3640 & 2.3601 & 2.3268 & 2.1167 & 1.9270 & 1.6534 & 1.4256 \\
\hline 0.5 & 0.05 & 63 & 2.4224 & 2.4283 & 2.4303 & 2.4219 & 2.3871 & 2.1630 & 1.9599 & 1.6683 & 1.4350 \\
\hline 0.5 & 0.05 & 72 & 2.4605 & 2.4664 & 2.4689 & 2.4658 & 2.4291 & 2.1948 & 1.9766 & 1.6799 & 1.4428 \\
\hline 0.5 & 0.05 & 81 & 2.4911 & 2.4971 & 2.4999 & 2.5046 & 2.4665 & 2.2245 & 1.9811 & 1.6896 & 1.4513 \\
\hline 0.5 & 0.05 & 90 & 2.4975 & 2.5035 & 2.5065 & 2.5074 & 2.4689 & 2.2253 & 1.9781 & 1.6816 & 1.4527 \\
\hline
\end{tabular}

Table A6. Normalized SIF for various number of cracks $(a / c=0.5, a / t=0.1)$

n

\begin{tabular}{|c|c|c|c|c|c|c|c|c|c|c|}
\hline $\mathrm{a} / \mathrm{c}$ & $\mathrm{a} / \mathrm{t}$ & $\mathrm{phi}$ & 1 & 2 & 4 & 8 & 16 & 20 & 32 & 40 \\
\hline & & & & & & & & & & \\
\hline 0.5 & 0.10 & 0 & 1.9915 & 1.9962 & 2.0321 & 1.9699 & 1.8938 & 1.8033 & 1.5755 & 1.4466 \\
\hline 0.5 & 0.10 & 9 & 1.9197 & 1.9278 & 1.9396 & 1.9020 & 1.8030 & 1.7218 & 1.4941 & 1.3703 \\
\hline 0.5 & 0.10 & 18 & 1.9543 & 1.9579 & 1.9644 & 1.9314 & 1.8163 & 1.7378 & 1.4984 & 1.3702 \\
\hline 0.5 & 0.10 & 27 & 2.0278 & 2.0310 & 2.0376 & 2.0030 & 1.8744 & 1.7859 & 1.5330 & 1.4044 \\
\hline 0.5 & 0.10 & 36 & 2.1022 & 2.1090 & 2.1181 & 2.0793 & 1.9425 & 1.8456 & 1.5768 & 1.4401 \\
\hline 0.5 & 0.10 & 45 & 2.1689 & 2.1793 & 2.1922 & 2.1478 & 2.0058 & 1.9004 & 1.6119 & 1.4630 \\
\hline 0.5 & 0.10 & 54 & 2.2275 & 2.2354 & 2.2527 & 2.2021 & 2.0517 & 1.9467 & 1.6357 & 1.4815 \\
\hline 0.5 & 0.10 & 63 & 2.2732 & 2.2796 & 2.3002 & 2.2449 & 2.0803 & 1.9876 & 1.6483 & 1.4898 \\
\hline 0.5 & 0.10 & 72 & 2.3053 & 2.3117 & 2.3343 & 2.2758 & 2.1055 & 2.0042 & 1.6567 & 1.4976 \\
\hline 0.5 & 0.10 & 81 & 2.3288 & 2.3332 & 2.3543 & 2.2966 & 2.1143 & 2.0147 & 1.6655 & 1.5087 \\
\hline 0.5 & 0.10 & 90 & 2.3201 & 2.3295 & 2.3559 & 2.2928 & 2.0893 & 2.0059 & 1.6715 & 1.5152 \\
\hline
\end{tabular}


Table A7. Normalized SIF for various number of cracks $(a / c=0.5, a / t=0.15)$

$\mathbf{n}$

\begin{tabular}{|c|c|c|c|c|c|c|c|c|}
\hline $\mathrm{a} / \mathrm{c}$ & $\mathrm{a} / \mathrm{t}$ & $\mathrm{phi}$ & 1 & 2 & 4 & 8 & 16 & 32 \\
\hline & & & & & & & & \\
\hline 0.5 & 0.15 & 0 & 2.0406 & 2.0454 & 2.0380 & 1.9751 & 1.7829 & 1.3928 \\
\hline 0.5 & 0.15 & 9 & 1.9385 & 1.9477 & 1.9471 & 1.8809 & 1.6914 & 1.3246 \\
\hline 0.5 & 0.15 & 18 & 1.9577 & 1.9633 & 1.9565 & 1.8959 & 1.6953 & 1.3218 \\
\hline 0.5 & 0.15 & 27 & 2.0114 & 2.0216 & 2.0146 & 1.9516 & 1.7314 & 1.3501 \\
\hline 0.5 & 0.15 & 36 & 2.0796 & 2.0853 & 2.0781 & 2.0122 & 1.7798 & 1.3809 \\
\hline 0.5 & 0.15 & 45 & 2.1412 & 2.1500 & 2.1428 & 2.0735 & 1.8294 & 1.4006 \\
\hline 0.5 & 0.15 & 54 & 2.2130 & 2.2205 & 2.2131 & 2.1401 & 1.8659 & 1.4119 \\
\hline 0.5 & 0.15 & 63 & 2.2677 & 2.2774 & 2.2699 & 2.1935 & 1.8860 & 1.4154 \\
\hline 0.5 & 0.15 & 72 & 2.3095 & 2.3164 & 2.3089 & 2.2299 & 1.9109 & 1.4198 \\
\hline 0.5 & 0.15 & 81 & 2.3258 & 2.3374 & 2.3299 & 2.2493 & 1.9281 & 1.4289 \\
\hline 0.5 & 0.15 & 90 & 2.3228 & 2.3297 & 2.3222 & 2.2416 & 1.9312 & 1.4332 \\
\hline
\end{tabular}

Table A8. Normalized SIF for various number of cracks $(a / c=0.5, a / t=0.2)$

$\mathbf{n}$

\begin{tabular}{|l|l|l|l|l|l|l|l|l|}
\hline $\mathrm{a} / \mathrm{c}$ & $\mathrm{a} / \mathrm{t}$ & $\mathrm{phi}$ & 1 & 2 & 4 & 8 & 16 & 20 \\
\hline & & & & & & & & \\
\hline 0.5 & 0.20 & 0 & 2.0057 & 2.0343 & 2.0092 & 1.9060 & 1.6225 & 1.5029 \\
\hline 0.5 & 0.20 & 9 & 1.9090 & 1.9239 & 1.9122 & 1.8141 & 1.5310 & 1.4183 \\
\hline 0.5 & 0.20 & 18 & 1.9229 & 1.9374 & 1.9261 & 1.8260 & 1.5255 & 1.4067 \\
\hline 0.5 & 0.20 & 27 & 1.9722 & 1.9862 & 1.9756 & 1.8698 & 1.5520 & 1.4305 \\
\hline 0.5 & 0.20 & 36 & 2.0301 & 2.0444 & 2.0337 & 1.9204 & 1.5871 & 1.4630 \\
\hline 0.5 & 0.20 & 45 & 2.0904 & 2.1048 & 2.0944 & 1.9725 & 1.6093 & 1.4845 \\
\hline 0.5 & 0.20 & 54 & 2.1481 & 2.1625 & 2.1526 & 2.0216 & 1.6186 & 1.4971 \\
\hline 0.5 & 0.20 & 63 & 2.1925 & 2.2068 & 2.1974 & 2.0584 & 1.6216 & 1.5020 \\
\hline 0.5 & 0.20 & 72 & 2.2299 & 2.2443 & 2.2356 & 2.0898 & 1.6258 & 1.5085 \\
\hline 0.5 & 0.20 & 81 & 2.2556 & 2.2701 & 2.2619 & 2.1116 & 1.6380 & 1.5189 \\
\hline 0.5 & 0.20 & 90 & 2.2591 & 2.2736 & 2.2656 & 2.1142 & 1.6444 & 1.5246 \\
\hline
\end{tabular}


Table A9. Normalized SIF for various number of cracks $(a / c=0.5, a / t=0.4)$

n

\begin{tabular}{|c|c|c|c|c|c|c|}
\hline $\mathrm{a} / \mathrm{c}$ & $\mathrm{a} / \mathrm{t}$ & $\mathrm{phi}$ & 1 & 2 & 4 & 8 \\
\hline & & & & & & \\
\hline 0.5 & 0.40 & 0 & 2.0755 & 2.1568 & 2.0840 & 1.7807 \\
\hline 0.5 & 0.40 & 9 & 1.9503 & 2.0224 & 1.9569 & 1.6672 \\
\hline 0.5 & 0.40 & 18 & 1.9272 & 1.9944 & 1.9356 & 1.6454 \\
\hline 0.5 & 0.40 & 27 & 1.9473 & 2.0114 & 1.9576 & 1.6664 \\
\hline 0.5 & 0.40 & 36 & 1.9834 & 2.0453 & 1.9966 & 1.7086 \\
\hline 0.5 & 0.40 & 45 & 2.0254 & 2.0845 & 2.0409 & 1.7466 \\
\hline 0.5 & 0.40 & 54 & 2.0726 & 2.1297 & 2.0910 & 1.7748 \\
\hline 0.5 & 0.40 & 63 & 2.1088 & 2.1649 & 2.1308 & 1.7984 \\
\hline 0.5 & 0.40 & 72 & 2.1351 & 2.1909 & 2.1609 & 1.8205 \\
\hline 0.5 & 0.40 & 81 & 2.1540 & 2.2088 & 2.1815 & 1.8393 \\
\hline 0.5 & 0.40 & 90 & 2.1422 & 2.1964 & 2.1705 & 1.8512 \\
\hline
\end{tabular}

Table A10. Normalized SIF for various number of cracks $(a / c=0.5, a / t=0.6)$

n

\begin{tabular}{|c|c|c|c|c|c|c|}
\hline $\mathrm{a} / \mathrm{c}$ & $\mathrm{a} / \mathrm{t}$ & $\mathrm{phi}$ & 1 & 2 & 3 & 6 \\
\hline & & & & & & \\
\hline 0.5 & 0.60 & 0 & 2.2612 & 2.4362 & 2.4406 & 1.9698 \\
\hline 0.5 & 0.60 & 9 & 2.0855 & 2.2948 & 2.2444 & 1.8287 \\
\hline 0.5 & 0.60 & 18 & 2.0241 & 2.2175 & 2.1724 & 1.7960 \\
\hline 0.5 & 0.60 & 27 & 2.0224 & 2.1995 & 2.1697 & 1.8201 \\
\hline 0.5 & 0.60 & 36 & 2.0522 & 2.2146 & 2.2018 & 1.8804 \\
\hline 0.5 & 0.60 & 45 & 2.0924 & 2.2489 & 2.2471 & 1.9469 \\
\hline 0.5 & 0.60 & 54 & 2.1424 & 2.2894 & 2.3043 & 2.0101 \\
\hline 0.5 & 0.60 & 63 & 2.1809 & 2.3257 & 2.3488 & 2.0780 \\
\hline 0.5 & 0.60 & 72 & 2.2163 & 2.3605 & 2.3891 & 2.1527 \\
\hline 0.5 & 0.60 & 81 & 2.2449 & 2.3851 & 2.4212 & 2.1880 \\
\hline 0.5 & 0.60 & 90 & 2.2443 & 2.3811 & 2.4204 & 2.2055 \\
\hline
\end{tabular}


Table A11. Normalized SIF for various number of cracks $(a / c=1, a / t=0.05)$

n

\begin{tabular}{|l|l|l|l|l|l|l|l|l|l|}
\hline $\mathrm{a} / \mathrm{c}$ & $\mathrm{a} / \mathrm{t}$ & $\mathrm{phi}$ & 1 & 2 & 4 & 8 & 16 & 32 & 64 \\
\hline & & & & & & & & & \\
\hline 1.0 & 0.05 & 0 & 2.7961 & 2.8035 & 2.8010 & 2.7996 & 2.7816 & 2.6503 & 2.1808 \\
\hline 1.0 & 0.05 & 9 & 2.7700 & 2.7773 & 2.7748 & 2.7735 & 2.7556 & 2.6155 & 2.1669 \\
\hline 1.0 & 0.05 & 18 & 2.6597 & 2.6666 & 2.6642 & 2.6630 & 2.6457 & 2.5131 & 2.0911 \\
\hline 1.0 & 0.05 & 27 & 2.5824 & 2.5890 & 2.5868 & 2.5856 & 2.5687 & 2.4569 & 2.0410 \\
\hline 1.0 & 0.05 & 36 & 2.5492 & 2.5557 & 2.5535 & 2.5524 & 2.5356 & 2.4186 & 2.0247 \\
\hline 1.0 & 0.05 & 45 & 2.5291 & 2.5355 & 2.5333 & 2.5322 & 2.5156 & 2.3840 & 2.0180 \\
\hline 1.0 & 0.05 & 54 & 2.5054 & 2.5156 & 2.5122 & 2.5110 & 2.4930 & 2.3727 & 2.0100 \\
\hline 1.0 & 0.05 & 63 & 2.4915 & 2.4978 & 2.4957 & 2.4946 & 2.4782 & 2.3529 & 2.0033 \\
\hline 1.0 & 0.05 & 72 & 2.4767 & 2.4829 & 2.4808 & 2.4797 & 2.4635 & 2.3439 & 1.9965 \\
\hline 1.0 & 0.05 & 81 & 2.4789 & 2.4850 & 2.4830 & 2.4819 & 2.4657 & 2.3413 & 2.0011 \\
\hline 1.0 & 0.05 & 90 & 2.4724 & 2.4785 & 2.4765 & 2.4754 & 2.4592 & 2.3281 & 1.9971 \\
\hline
\end{tabular}

Table A12. Normalized SIF for various number of cracks $(a / c=1, a / t=0.1)$

$\mathbf{n}$

\begin{tabular}{|c|c|c|c|c|c|c|c|c|c|}
\hline $\mathrm{a} / \mathrm{c}$ & $\mathrm{a} / \mathrm{t}$ & $\mathrm{phi}$ & 1 & 2 & 4 & 8 & 16 & 32 & 45 \\
\hline & & & & & & & & & \\
\hline 1.0 & 0.10 & 0 & 2.7374 & 2.7424 & 2.7433 & 2.7158 & 2.6211 & 2.1707 & 1.8679 \\
\hline 1.0 & 0.10 & 9 & 2.6847 & 2.6895 & 2.6908 & 2.6636 & 2.5699 & 2.1342 & 1.8413 \\
\hline 1.0 & 0.10 & 18 & 2.5649 & 2.5694 & 2.5704 & 2.5449 & 2.4544 & 2.0485 & 1.7781 \\
\hline 1.0 & 0.10 & 27 & 2.5068 & 2.5111 & 2.5119 & 2.4879 & 2.3978 & 2.0113 & 1.7583 \\
\hline 1.0 & 0.10 & 36 & 2.4622 & 2.4664 & 2.4670 & 2.4446 & 2.3545 & 1.9839 & 1.7425 \\
\hline 1.0 & 0.10 & 45 & 2.4332 & 2.4373 & 2.4377 & 2.4167 & 2.3265 & 1.9681 & 1.7329 \\
\hline 1.0 & 0.10 & 54 & 2.4116 & 2.4151 & 2.4153 & 2.3955 & 2.3055 & 1.9567 & 1.7344 \\
\hline 1.0 & 0.10 & 63 & 2.3938 & 2.3977 & 2.3976 & 2.3787 & 2.2893 & 1.9480 & 1.7287 \\
\hline 1.0 & 0.10 & 72 & 2.3891 & 2.3929 & 2.3927 & 2.3744 & 2.2852 & 1.9480 & 1.7220 \\
\hline 1.0 & 0.10 & 81 & 2.3879 & 2.3917 & 2.3913 & 2.3733 & 2.2843 & 1.9430 & 1.7173 \\
\hline 1.0 & 0.10 & 90 & 2.3772 & 2.3810 & 2.3806 & 2.3628 & 2.2742 & 1.9442 & 1.7171 \\
\hline
\end{tabular}


Table A13. Normalized SIF for various number of cracks $(a / c=1, a / t=0.15)$

n

\begin{tabular}{|c|c|c|c|c|c|c|c|c|}
\hline $\mathrm{a} / \mathrm{c}$ & $\mathrm{a} / \mathrm{t}$ & $\mathrm{phi}$ & 1 & 2 & 4 & 8 & 16 & 32 \\
\hline & & & & & & & & \\
\hline 1.0 & 0.15 & 0 & 2.7066 & 2.7135 & 2.7091 & 2.6448 & 2.4193 & 1.8771 \\
\hline 1.0 & 0.15 & 9 & 2.6462 & 2.6532 & 2.6486 & 2.5884 & 2.3657 & 1.8483 \\
\hline 1.0 & 0.15 & 18 & 2.5133 & 2.5198 & 2.5160 & 2.4588 & 2.2492 & 1.7768 \\
\hline 1.0 & 0.15 & 27 & 2.4443 & 2.4503 & 2.4471 & 2.3930 & 2.1903 & 1.7474 \\
\hline 1.0 & 0.15 & 36 & 2.3915 & 2.3973 & 2.3944 & 2.3460 & 2.1463 & 1.7265 \\
\hline 1.0 & 0.15 & 45 & 2.3538 & 2.3593 & 2.3567 & 2.3121 & 2.1157 & 1.7132 \\
\hline 1.0 & 0.15 & 54 & 2.3268 & 2.3321 & 2.3297 & 2.2875 & 2.0947 & 1.7046 \\
\hline 1.0 & 0.15 & 63 & 2.3053 & 2.3105 & 2.3082 & 2.2675 & 2.0781 & 1.6972 \\
\hline 1.0 & 0.15 & 72 & 2.2974 & 2.3026 & 2.3003 & 2.2604 & 2.0732 & 1.6966 \\
\hline 1.0 & 0.15 & 81 & 2.2956 & 2.3071 & 2.2985 & 2.2590 & 2.0728 & 1.6981 \\
\hline 1.0 & 0.15 & 90 & 2.2790 & 2.2840 & 2.2818 & 2.2473 & 2.0583 & 1.6871 \\
\hline
\end{tabular}

Table A14. Normalized SIF for various number of cracks $(a / c=1, a / t=0.2)$

n

\begin{tabular}{|c|c|c|c|c|c|c|c|c|}
\hline $\mathrm{a} / \mathrm{c}$ & $\mathrm{a} / \mathrm{t}$ & $\mathrm{phi}$ & 1 & 2 & 4 & 8 & 16 & 20 \\
\hline & & & & & & & & \\
\hline 1.0 & 0.20 & 0 & 2.6505 & 2.6621 & 2.6636 & 2.5738 & 2.2006 & 2.0008 \\
\hline 1.0 & 0.20 & 9 & 2.6001 & 2.6111 & 2.6093 & 2.5215 & 2.1594 & 1.9699 \\
\hline 1.0 & 0.20 & 18 & 2.4677 & 2.4778 & 2.4744 & 2.3917 & 2.0562 & 1.8861 \\
\hline 1.0 & 0.20 & 27 & 2.3684 & 2.3778 & 2.3725 & 2.2941 & 1.9811 & 1.8271 \\
\hline 1.0 & 0.20 & 36 & 2.3093 & 2.3181 & 2.3112 & 2.2361 & 1.9393 & 1.7968 \\
\hline 1.0 & 0.20 & 45 & 2.2671 & 2.2754 & 2.2678 & 2.1958 & 1.9114 & 1.7767 \\
\hline 1.0 & 0.20 & 54 & 2.2467 & 2.2547 & 2.2467 & 2.1770 & 1.9003 & 1.7696 \\
\hline 1.0 & 0.20 & 63 & 2.2251 & 2.2329 & 2.2250 & 2.1576 & 1.8866 & 1.7577 \\
\hline 1.0 & 0.20 & 72 & 2.1973 & 2.2048 & 2.1974 & 2.1320 & 1.8658 & 1.7379 \\
\hline 1.0 & 0.20 & 81 & 2.2029 & 2.2104 & 2.2032 & 2.1384 & 1.8718 & 1.7424 \\
\hline 1.0 & 0.20 & 90 & 2.1982 & 2.2057 & 2.1986 & 2.1343 & 1.8683 & 1.7387 \\
\hline
\end{tabular}


Table A15. Normalized SIF for various number of cracks $(a / c=1, a / t=0.4)$

$\mathbf{n}$

\begin{tabular}{|c|c|c|c|c|c|c|c|}
\hline $\mathrm{a} / \mathrm{c}$ & $\mathrm{a} / \mathrm{t}$ & $\mathrm{phi}$ & 1 & 2 & 4 & 8 & 10 \\
\hline & & & & & & & \\
\hline 1.0 & 0.40 & 0 & 2.6134 & 2.6547 & 2.6248 & 2.3135 & 2.1342 \\
\hline 1.0 & 0.40 & 9 & 2.5157 & 2.5530 & 2.5260 & 2.2346 & 2.0692 \\
\hline 1.0 & 0.40 & 18 & 2.3638 & 2.3963 & 2.3732 & 2.1098 & 1.9610 \\
\hline 1.0 & 0.40 & 27 & 2.2558 & 2.2845 & 2.2651 & 2.0267 & 1.8865 \\
\hline 1.0 & 0.40 & 36 & 2.1898 & 2.2154 & 2.1995 & 1.9735 & 1.8438 \\
\hline 1.0 & 0.40 & 45 & 2.1398 & 2.1630 & 2.1502 & 1.9368 & 1.8154 \\
\hline 1.0 & 0.40 & 54 & 2.1008 & 2.1221 & 2.1119 & 1.9088 & 1.8018 \\
\hline 1.0 & 0.40 & 63 & 2.0806 & 2.1006 & 2.0925 & 1.8966 & 1.7945 \\
\hline 1.0 & 0.40 & 72 & 2.0608 & 2.0797 & 2.0731 & 1.8832 & 1.7795 \\
\hline 1.0 & 0.40 & 81 & 2.0440 & 2.0622 & 2.0566 & 1.8708 & 1.7679 \\
\hline 1.0 & 0.40 & 90 & 2.0348 & 2.0528 & 2.0476 & 1.8635 & 1.7638 \\
\hline
\end{tabular}

Table A16. Normalized SIF for various number of $\operatorname{cracks}(a / c=1, a / t=0.6)$

n

\begin{tabular}{|c|c|c|c|c|c|c|}
\hline $\mathrm{a} / \mathrm{c}$ & $\mathrm{a} / \mathrm{t}$ & $\mathrm{phi}$ & 1 & 2 & 4 & 6 \\
\hline & & & & & & \\
\hline 1.0 & 0.60 & 0 & 2.6747 & 2.7751 & 2.7128 & 2.4460 \\
\hline 1.0 & 0.60 & 9 & 2.5270 & 2.6133 & 2.5618 & 2.3258 \\
\hline 1.0 & 0.60 & 18 & 2.3367 & 2.4078 & 2.3695 & 2.1716 \\
\hline 1.0 & 0.60 & 27 & 2.2380 & 2.2948 & 2.2687 & 2.0963 \\
\hline 1.0 & 0.60 & 36 & 2.1568 & 2.2073 & 2.1926 & 2.0423 \\
\hline 1.0 & 0.60 & 45 & 2.1018 & 2.1449 & 2.1404 & 2.0082 \\
\hline 1.0 & 0.60 & 54 & 2.0603 & 2.0974 & 2.1016 & 1.9847 \\
\hline 1.0 & 0.60 & 63 & 2.0379 & 2.0706 & 2.0817 & 1.9767 \\
\hline 1.0 & 0.60 & 72 & 2.0144 & 2.0439 & 2.0600 & 1.9643 \\
\hline 1.0 & 0.60 & 81 & 2.0025 & 2.0300 & 2.0492 & 1.9591 \\
\hline 1.0 & 0.60 & 90 & 1.9938 & 2.0205 & 2.0408 & 1.9530 \\
\hline
\end{tabular}


Table A17. Normalized SIF for various number of cracks $(a / c=1.5, a / t=0.05)$

n

\begin{tabular}{|r|c|c|c|c|c|c|c|c|c|c|}
\hline phi & 1 & 2 & 4 & 8 & 16 & 32 & 45 & 64 & 90 & 180 \\
\hline & & & & & & & & & & \\
\hline 0 & 3.3147 & 3.3282 & 3.3261 & 3.3255 & 3.3144 & 3.2239 & 3.0772 & 2.7657 & 2.3863 & 1.6271 \\
\hline 9 & 3.2763 & 3.2896 & 3.2875 & 3.2868 & 3.2758 & 3.1869 & 3.0440 & 2.7815 & 2.3871 & 1.6588 \\
\hline 18 & 3.0896 & 3.1020 & 3.1000 & 3.0994 & 3.0888 & 3.0061 & 2.8748 & 2.6181 & 2.2912 & 1.6221 \\
\hline 27 & 2.9824 & 2.9942 & 2.9923 & 2.9915 & 2.9813 & 2.9029 & 2.7803 & 2.5404 & 2.2626 & 1.6231 \\
\hline 36 & 2.8648 & 2.8761 & 2.8743 & 2.8734 & 2.8635 & 2.7899 & 2.6766 & 2.4626 & 2.2038 & 1.6190 \\
\hline 45 & 2.7673 & 2.7780 & 2.7763 & 2.7753 & 2.7657 & 2.6963 & 2.5912 & 2.3853 & 2.1646 & 1.6081 \\
\hline 54 & 2.6703 & 2.6806 & 2.6790 & 2.6778 & 2.6685 & 2.6032 & 2.5056 & 2.3089 & 2.1192 & 1.5996 \\
\hline 63 & 2.5589 & 2.5687 & 2.5672 & 2.5660 & 2.5570 & 2.4958 & 2.4051 & 2.2519 & 2.0551 & 1.5783 \\
\hline 72 & 2.4639 & 2.4732 & 2.4718 & 2.4705 & 2.4619 & 2.4040 & 2.3192 & 2.1879 & 1.9959 & 1.5554 \\
\hline 81 & 2.4082 & 2.4174 & 2.4159 & 2.4147 & 2.4063 & 2.3503 & 2.2688 & 2.1291 & 1.9612 & 1.5386 \\
\hline 90 & 2.3734 & 2.3824 & 2.3810 & 2.3798 & 2.3714 & 2.3165 & 2.2367 & 2.1052 & 1.9365 & 1.5266 \\
\hline
\end{tabular}

Table A18. Normalized SIF for various number of $\operatorname{cracks}(a / c=1.5, a / t=0.1)$

$\mathbf{n}$

\begin{tabular}{|c|c|c|c|c|c|c|c|c|c|}
\hline phi & 1 & 2 & 4 & 8 & 16 & 32 & 50 & 64 & 100 \\
\hline & & & & & & & & & \\
\hline 0 & 3.2353 & 3.2413 & 3.2358 & 3.2367 & 3.1688 & 2.7573 & 2.2491 & 1.9297 & 1.5292 \\
\hline 9 & 3.1965 & 3.2023 & 3.1970 & 3.1977 & 3.1299 & 2.7335 & 2.2535 & 1.9730 & 1.5578 \\
\hline 18 & 3.0068 & 3.0121 & 3.0074 & 3.0077 & 2.9435 & 2.5816 & 2.1643 & 1.9331 & 1.5327 \\
\hline 27 & 2.8909 & 2.8959 & 2.8916 & 2.8916 & 2.8293 & 2.5032 & 2.1262 & 1.9067 & 1.5276 \\
\hline 36 & 2.7724 & 2.7771 & 2.7732 & 2.7726 & 2.7126 & 2.4177 & 2.0825 & 1.8893 & 1.5219 \\
\hline 45 & 2.6720 & 2.6764 & 2.6728 & 2.6715 & 2.6138 & 2.3461 & 2.0460 & 1.8563 & 1.5101 \\
\hline 54 & 2.5642 & 2.5684 & 2.5651 & 2.5629 & 2.5081 & 2.2653 & 1.9954 & 1.8286 & 1.4950 \\
\hline 63 & 2.4574 & 2.4614 & 2.4584 & 2.4555 & 2.4035 & 2.1820 & 1.9371 & 1.7992 & 1.4753 \\
\hline 72 & 2.3738 & 2.3776 & 2.3748 & 2.3714 & 2.3216 & 2.1157 & 1.8882 & 1.7624 & 1.4661 \\
\hline 81 & 2.3123 & 2.3160 & 2.3134 & 2.3097 & 2.2615 & 2.0657 & 1.8492 & 1.7317 & 1.4567 \\
\hline 90 & 2.2879 & 2.2916 & 2.2890 & 2.2852 & 2.2376 & 2.0455 & 1.8333 & 1.7171 & 1.4447 \\
\hline
\end{tabular}


Table A19. Normalized SIF for various number of cracks $(a / c=1.5, a / t=0.15)$

n

\begin{tabular}{|c|c|c|c|c|c|c|c|c|c|}
\hline $\mathrm{a} / \mathrm{c}$ & $\mathrm{a} / \mathrm{t}$ & $\mathrm{phi}$ & 1 & 2 & 4 & 8 & 16 & 32 & 64 \\
\hline & & & & & & & & & \\
\hline 1.5 & 0.15 & 0 & 3.1562 & 3.1560 & 3.1537 & 3.1234 & 2.9347 & 2.1397 & 1.5588 \\
\hline 1.5 & 0.15 & 9 & 3.1247 & 3.1326 & 3.1303 & 3.1002 & 2.9144 & 2.1552 & 1.5733 \\
\hline 1.5 & 0.15 & 18 & 2.9505 & 2.9518 & 2.9496 & 2.9213 & 2.7497 & 2.0757 & 1.5448 \\
\hline 1.5 & 0.15 & 27 & 2.8215 & 2.8250 & 2.8226 & 2.7956 & 2.6364 & 2.0316 & 1.5386 \\
\hline 1.5 & 0.15 & 36 & 2.7201 & 2.7192 & 2.7167 & 2.6909 & 2.5431 & 1.9988 & 1.5339 \\
\hline 1.5 & 0.15 & 45 & 2.5996 & 2.6089 & 2.6063 & 2.5818 & 2.4453 & 1.9566 & 1.5203 \\
\hline 1.5 & 0.15 & 54 & 2.5047 & 2.5043 & 2.5016 & 2.4785 & 2.3522 & 1.9111 & 1.5067 \\
\hline 1.5 & 0.15 & 63 & 2.4140 & 2.4157 & 2.4128 & 2.3910 & 2.2728 & 1.8695 & 1.4921 \\
\hline 1.5 & 0.15 & 72 & 2.3304 & 2.3283 & 2.3254 & 2.3047 & 2.1935 & 1.8204 & 1.4726 \\
\hline 1.5 & 0.15 & 81 & 2.2616 & 2.2660 & 2.2631 & 2.2432 & 2.1366 & 1.7830 & 1.4490 \\
\hline 1.5 & 0.15 & 90 & 2.2323 & 2.2347 & 2.2346 & 2.2149 & 2.1102 & 1.7644 & 1.4414 \\
\hline
\end{tabular}

Table A20. Normalized SIF for various number of cracks $(a / c=1.5, a / t=0.2)$

n

\begin{tabular}{|c|c|c|c|c|c|c|c|c|c|c|}
\hline $\mathrm{a} / \mathrm{c}$ & $\mathrm{a} / \mathrm{t}$ & $\mathrm{phi}$ & 1 & 2 & 4 & 8 & 16 & 25 & 32 & 50 \\
\hline & & & & & & & & & & \\
\hline 1.5 & 0.20 & 0 & 3.0877 & 3.1036 & 3.0935 & 3.0371 & 2.7017 & 2.2321 & 1.9480 & 1.5534 \\
\hline 1.5 & 0.20 & 9 & 3.0645 & 3.0792 & 3.0696 & 3.0138 & 2.6873 & 2.2409 & 1.9748 & 1.5807 \\
\hline 1.5 & 0.20 & 18 & 2.8786 & 2.8911 & 2.8825 & 2.8308 & 2.5353 & 2.1432 & 1.9221 & 1.5554 \\
\hline 1.5 & 0.20 & 27 & 2.7584 & 2.7690 & 2.7612 & 2.7127 & 2.4426 & 2.0930 & 1.8842 & 1.5399 \\
\hline 1.5 & 0.20 & 36 & 2.6359 & 2.6447 & 2.6375 & 2.5925 & 2.3479 & 2.0381 & 1.8563 & 1.5228 \\
\hline 1.5 & 0.20 & 45 & 2.5279 & 2.5352 & 2.5287 & 2.4867 & 2.2646 & 1.9884 & 1.8146 & 1.5039 \\
\hline 1.5 & 0.20 & 54 & 2.4272 & 2.4334 & 2.4274 & 2.3881 & 2.1856 & 1.9373 & 1.7794 & 1.4837 \\
\hline 1.5 & 0.20 & 63 & 2.3299 & 2.3352 & 2.3296 & 2.2927 & 2.1068 & 1.8814 & 1.7443 & 1.4672 \\
\hline 1.5 & 0.20 & 72 & 2.2326 & 2.2371 & 2.2320 & 2.1972 & 2.0251 & 1.8177 & 1.7041 & 1.4540 \\
\hline 1.5 & 0.20 & 81 & 2.1819 & 2.1861 & 2.1812 & 2.1475 & 1.9828 & 1.7849 & 1.6703 & 1.4327 \\
\hline 1.5 & 0.20 & 90 & 2.1573 & 2.1613 & 2.1565 & 2.1234 & 1.9618 & 1.7678 & 1.6559 & 1.4219 \\
\hline
\end{tabular}


Table A21. Normalized SIF for various number of $\operatorname{cracks}(a / c=1.5, a / t=0.4)$

$\mathbf{n}$

\begin{tabular}{|c|c|c|c|c|c|c|c|c|}
\hline $\mathrm{a} / \mathrm{c}$ & $\mathrm{a} / \mathrm{t}$ & $\mathrm{phi}$ & 1 & 2 & 4 & 8 & 16 & 32 \\
\hline & & & & & & & & \\
\hline 1.5 & 0.40 & 0 & 3.0216 & 3.0498 & 3.0309 & 2.7818 & 2.0935 & 1.4704 \\
\hline 1.5 & 0.40 & 9 & 2.9506 & 2.9762 & 2.9587 & 2.7223 & 2.0796 & 1.4665 \\
\hline 1.5 & 0.40 & 18 & 2.7483 & 2.7702 & 2.7555 & 2.5457 & 1.9863 & 1.4190 \\
\hline 1.5 & 0.40 & 27 & 2.5815 & 2.6003 & 2.5884 & 2.4030 & 1.9142 & 1.3946 \\
\hline 1.5 & 0.40 & 36 & 2.4497 & 2.4660 & 2.4566 & 2.2924 & 1.8620 & 1.3766 \\
\hline 1.5 & 0.40 & 45 & 2.3395 & 2.3538 & 2.3466 & 2.2006 & 1.8188 & 1.3558 \\
\hline 1.5 & 0.40 & 54 & 2.2293 & 2.2419 & 2.2366 & 2.1067 & 1.7675 & 1.3359 \\
\hline 1.5 & 0.40 & 63 & 2.1248 & 2.1360 & 2.1322 & 2.0157 & 1.7120 & 1.3209 \\
\hline 1.5 & 0.40 & 72 & 2.0427 & 2.0528 & 2.0501 & 1.9433 & 1.6511 & 1.3098 \\
\hline 1.5 & 0.40 & 81 & 1.9801 & 1.9896 & 1.9875 & 1.8710 & 1.6257 & 1.2908 \\
\hline 1.5 & 0.40 & 90 & 1.9573 & 1.9667 & 1.9648 & 1.8666 & 1.6111 & 1.2828 \\
\hline
\end{tabular}

Table A22. Normalized SIF for various number of cracks $(a / c=1.5, a / t=0.6)$

\begin{tabular}{|c|c|c|c|c|c|c|c|c|c|}
\hline $\mathrm{g} / \mathrm{c}$ & $\mathrm{a} / \mathrm{t}$ & $\mathrm{phi}$ & 1 & 2 & 4 & 8 & 10 & 16 & 20 \\
\hline & & & & & & & & & \\
\hline 1.5 & 0.60 & 0 & 3.0077 & 3.0695 & 3.0352 & 2.5679 & 2.3297 & 1.8170 & 1.6716 \\
\hline 1.5 & 0.60 & 9 & 2.8676 & 2.9203 & 2.8917 & 2.4756 & 2.2614 & 1.8060 & 1.6410 \\
\hline 1.5 & 0.60 & 18 & 2.6404 & 2.6828 & 2.6624 & 2.3142 & 2.1316 & 1.7481 & 1.5684 \\
\hline 1.5 & 0.60 & 27 & 2.4916 & 2.5261 & 2.5137 & 2.2191 & 2.0602 & 1.7080 & 1.5352 \\
\hline 1.5 & 0.60 & 36 & 2.3546 & 2.3824 & 2.3776 & 2.1310 & 1.9935 & 1.6782 & 1.5087 \\
\hline 1.5 & 0.60 & 45 & 2.2448 & 2.2673 & 2.2690 & 2.0623 & 1.9426 & 1.6510 & 1.4938 \\
\hline 1.5 & 0.60 & 54 & 2.1368 & 2.1549 & 2.1618 & 1.9893 & 1.8510 & 1.6210 & 1.4727 \\
\hline 1.5 & 0.60 & 63 & 2.0276 & 2.0422 & 2.0530 & 1.9087 & 1.8177 & 1.5829 & 1.4490 \\
\hline 1.5 & 0.60 & 72 & 1.9506 & 1.9629 & 1.9762 & 1.8513 & 1.7692 & 1.5572 & 1.4374 \\
\hline 1.5 & 0.60 & 81 & 1.8950 & 1.9059 & 1.9205 & 1.8077 & 1.7313 & 1.5392 & 1.4234 \\
\hline 1.5 & 0.60 & 90 & 1.8758 & 1.8862 & 1.9013 & 1.7926 & 1.7182 & 1.5279 & 1.4127 \\
\hline
\end{tabular}




\section{APPENDIX B}

NORMALIZED STRESS INTENSITY FACTORS vs. $\phi$ 


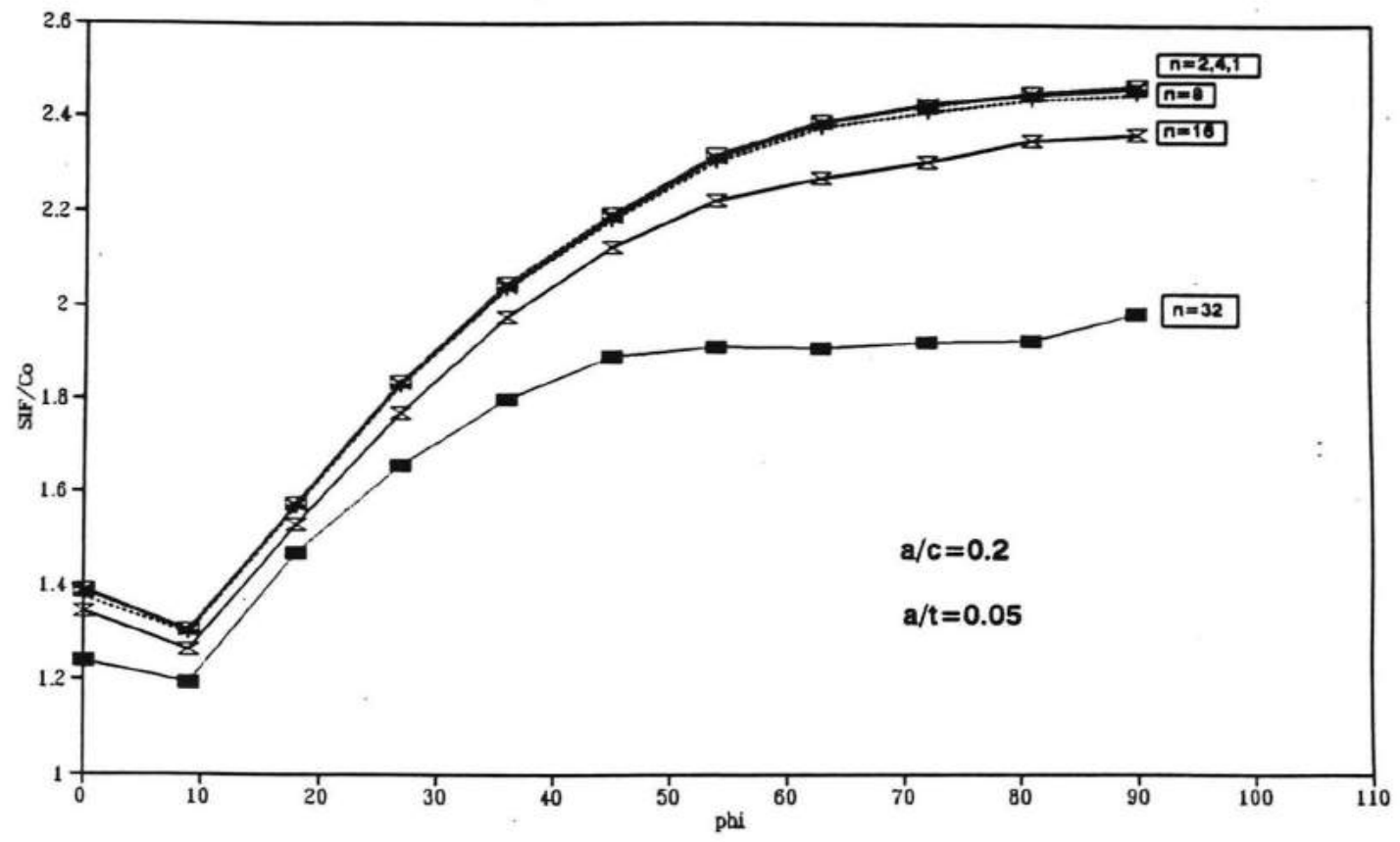

Fig.B.1 Normalized stress intensity factor vs. $\phi(a / c=0.2, a / t=0.05)$

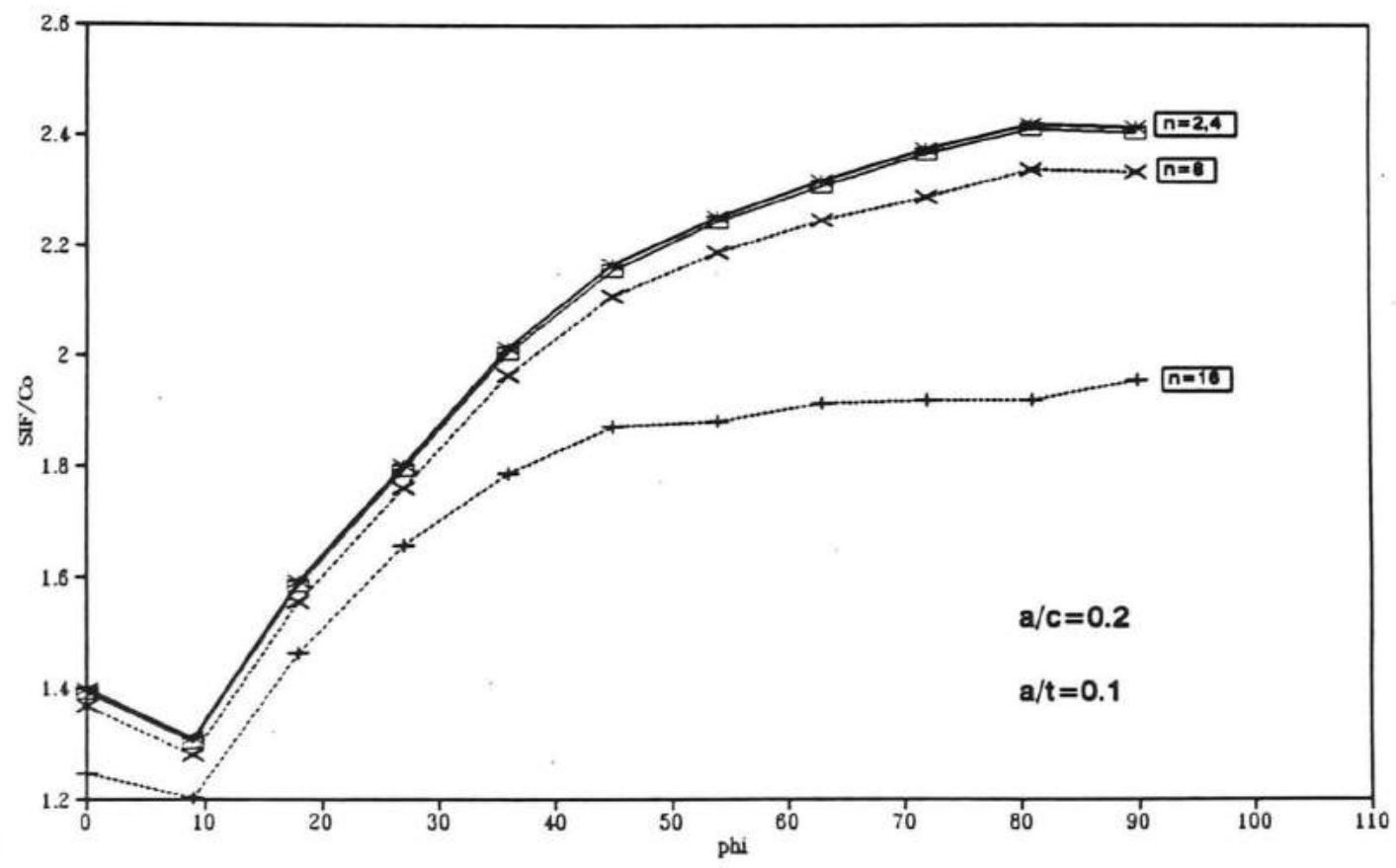

Fig.B.2 Normalized stress intensity factor vs. $\phi(a / c=0.2, a / t=0.1)$ 


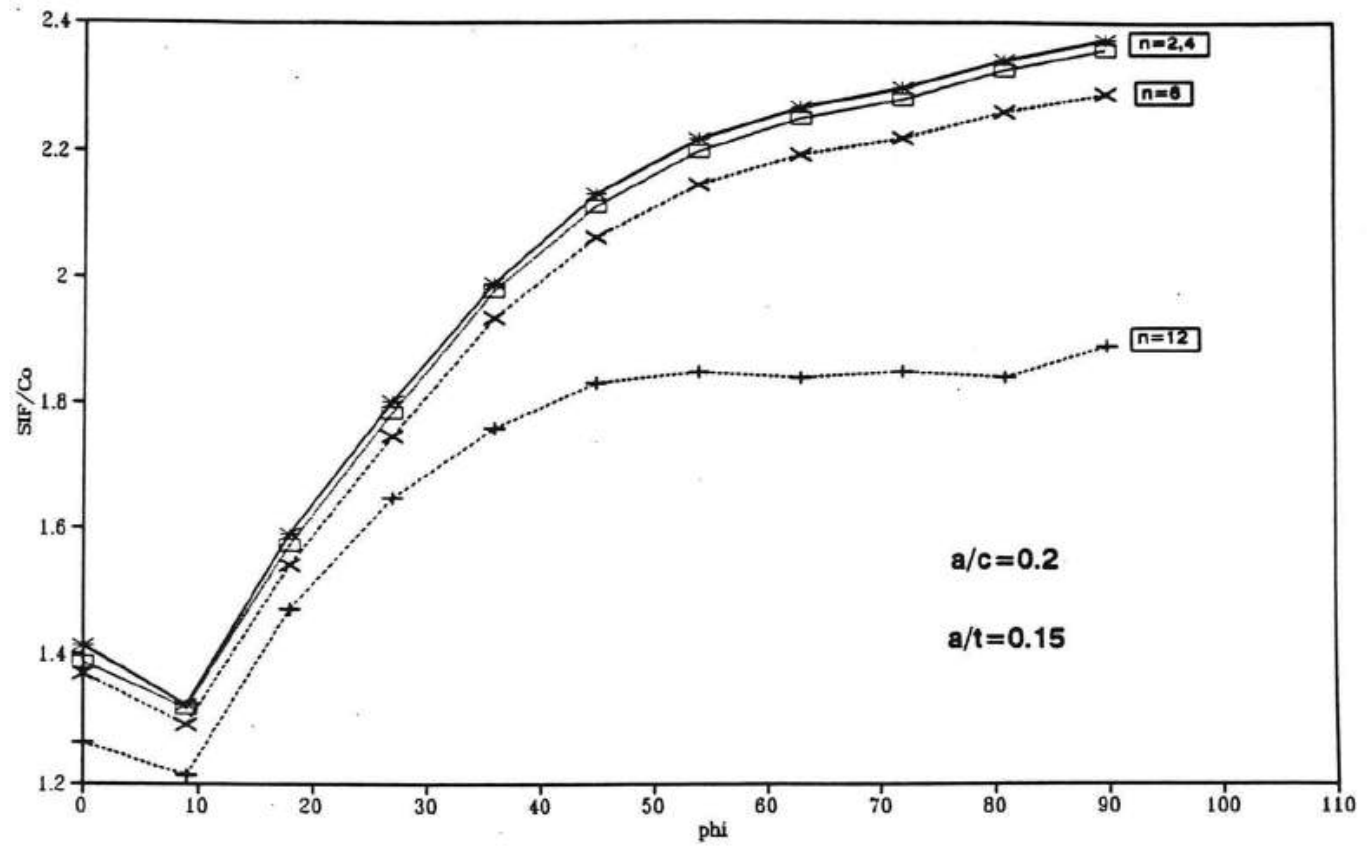

Fig. B.3 Normalized stress intensity factor vs. $\phi(a / c=0.2, a / t=0.15)$

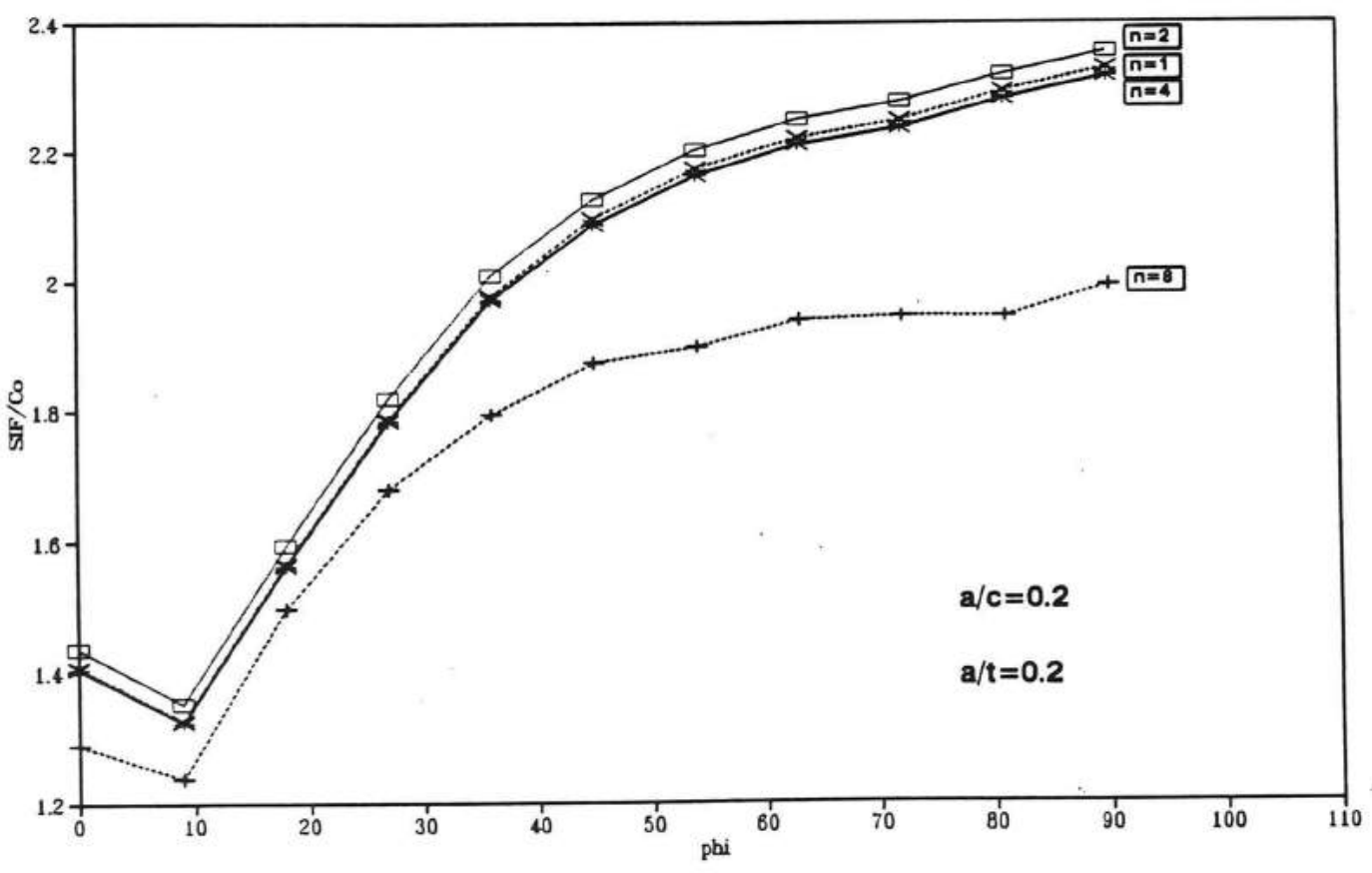

Fig. B.4 Normalized stress intensity factor vs. $\phi(a / c=0.2, a / t=0.2)$ 


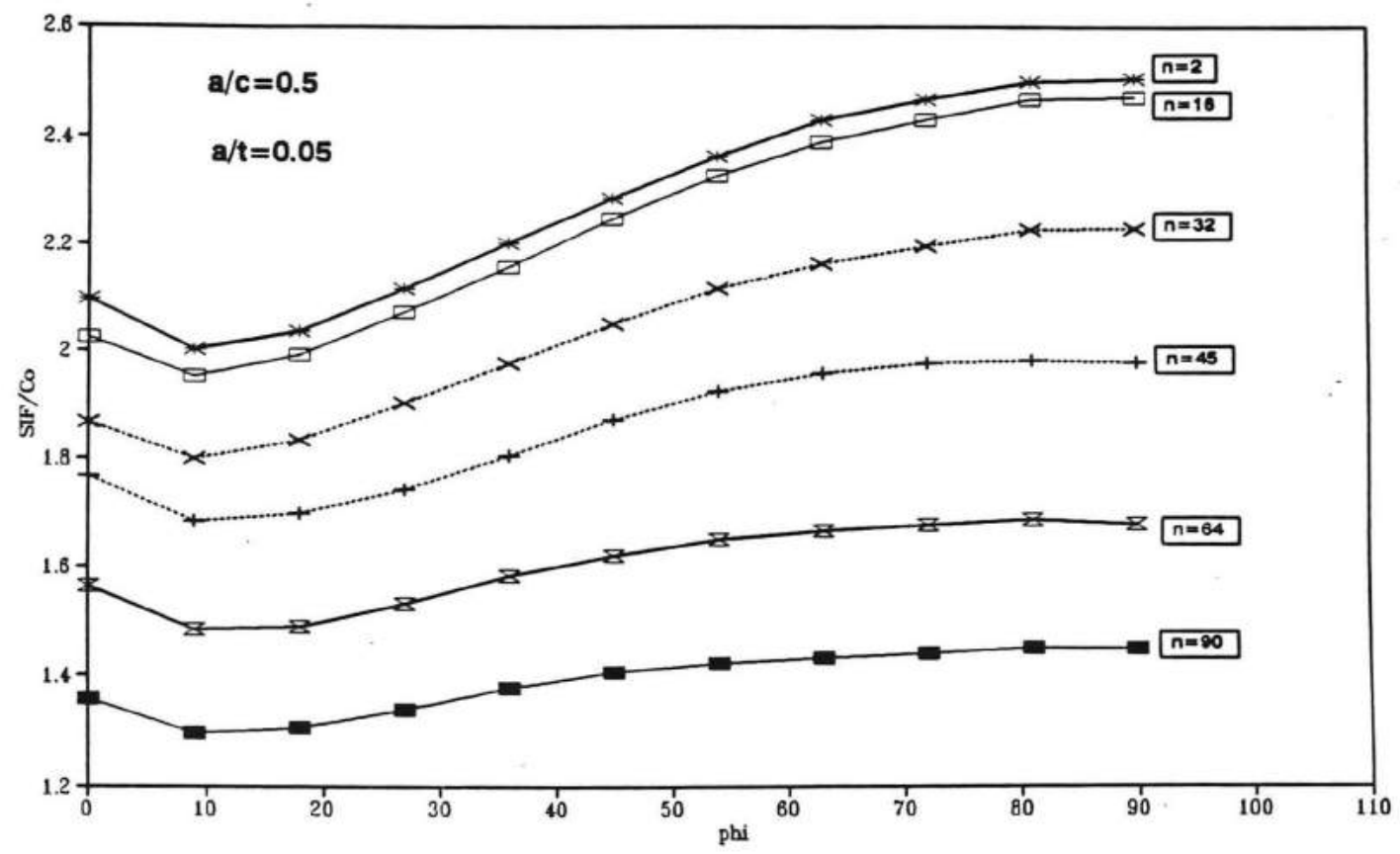

Fig.B.5 Normalized stress intensity factor vs. $\phi(a / c=0.5, a / t=0.05)$

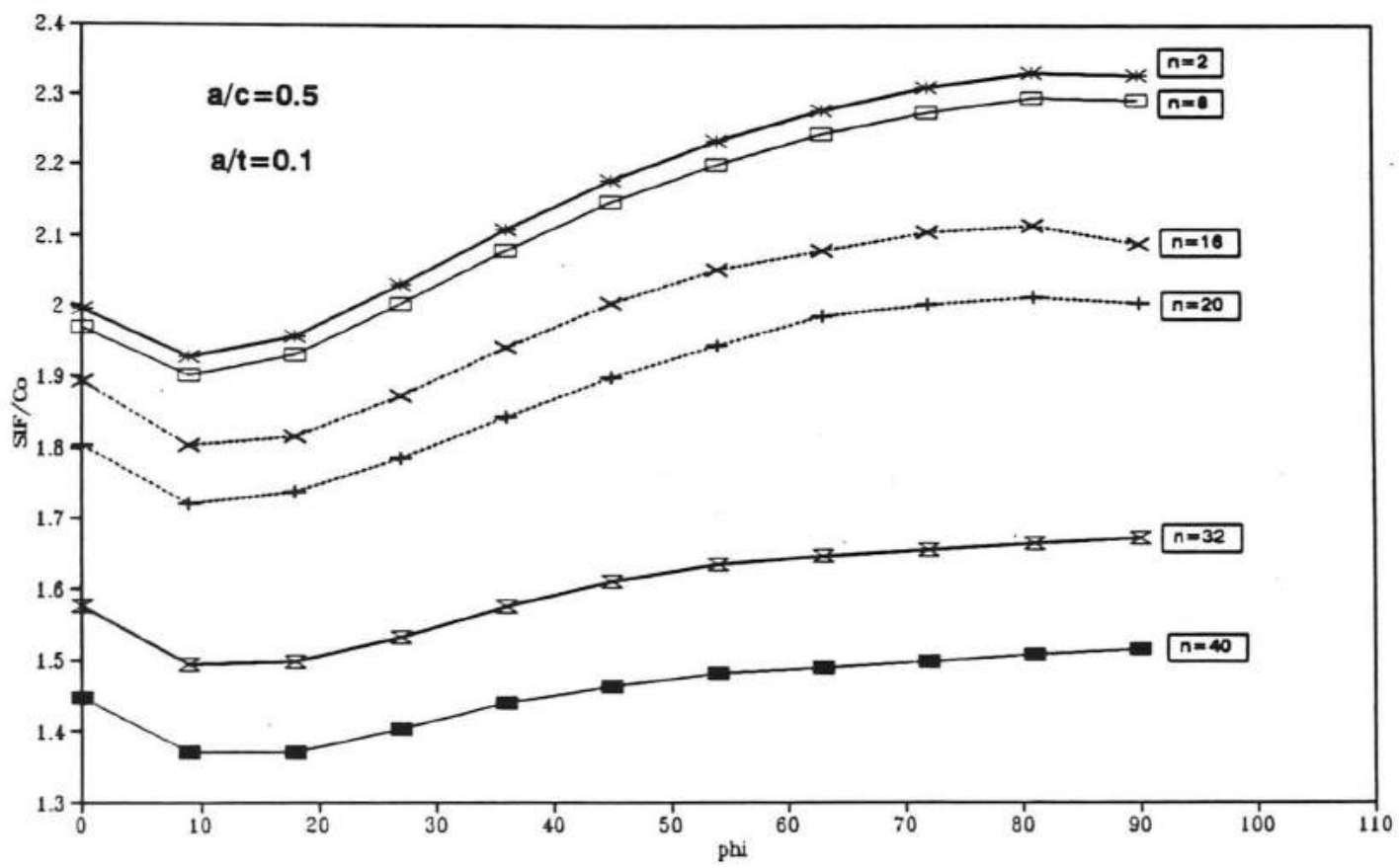

Fig.B.6 Normalized stress intensity factor vs. $\phi(a / c=0.5, a / t=0.1)$ 


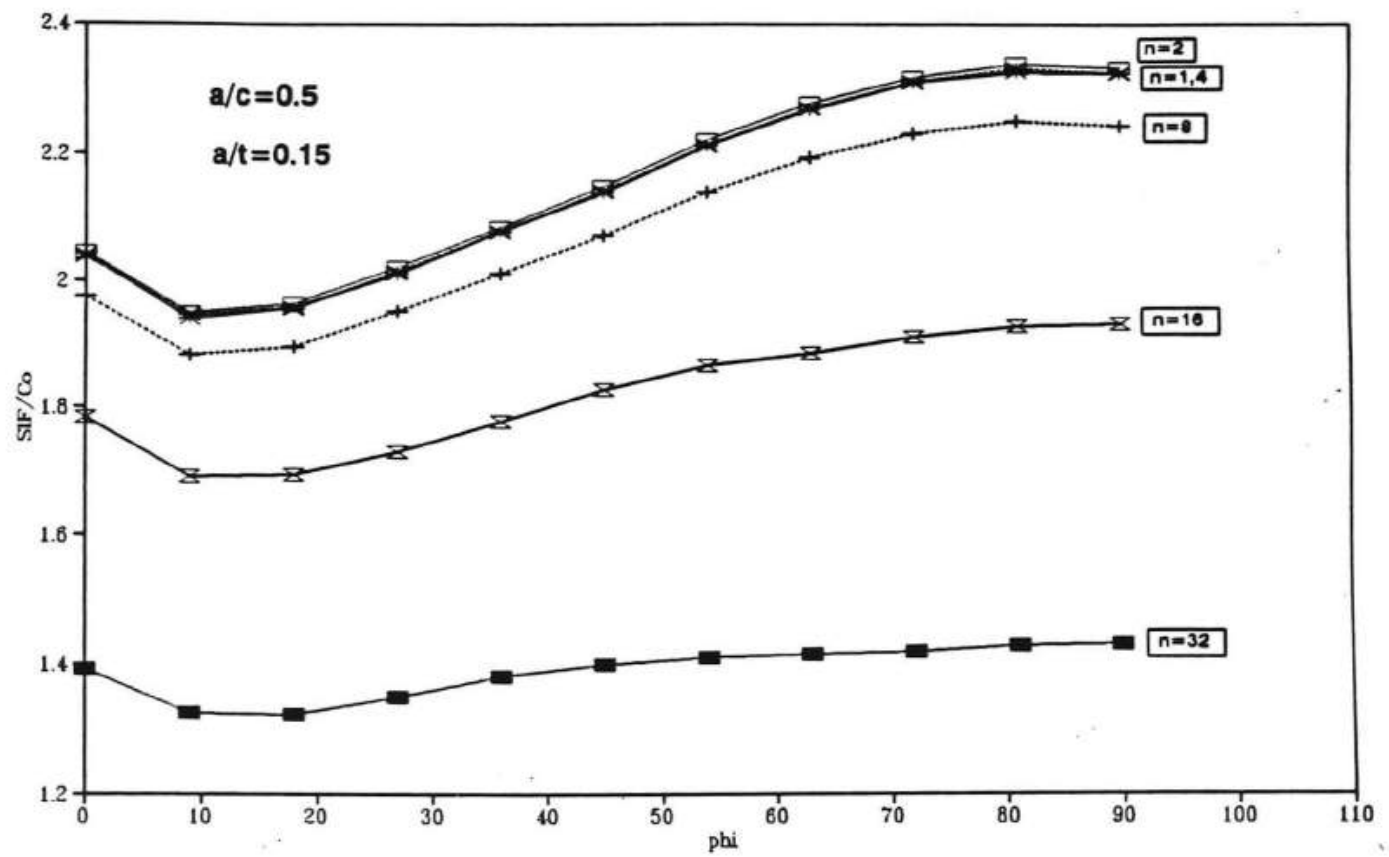

Fig.B.7 Normalized stress intensity factor vs. $\phi(a / c=0.5, a / t=0.15)$

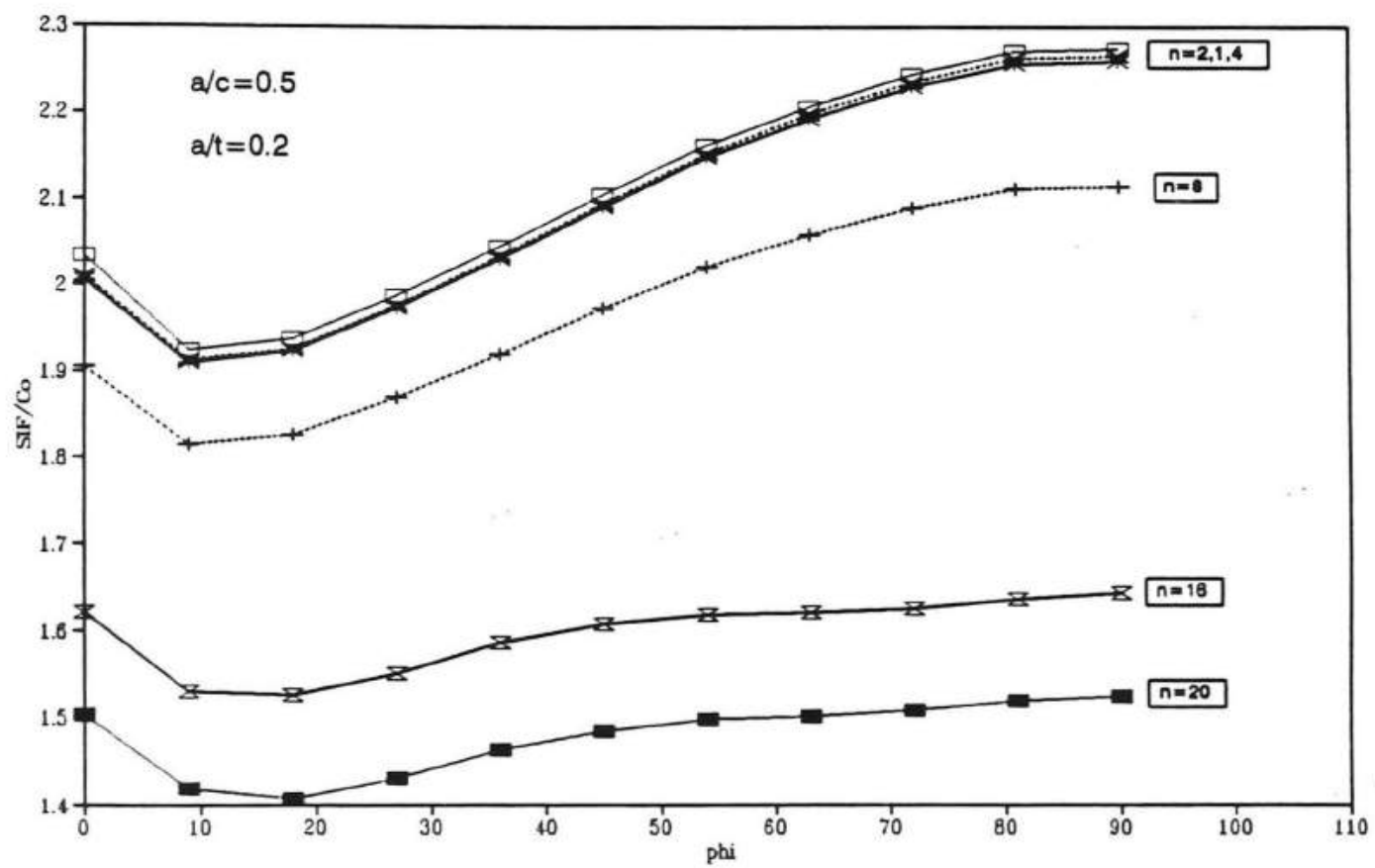

Fig.B.8 Normalized stress intensity factor vs. $\phi(a / c=0.5, a / t=0.2)$ 


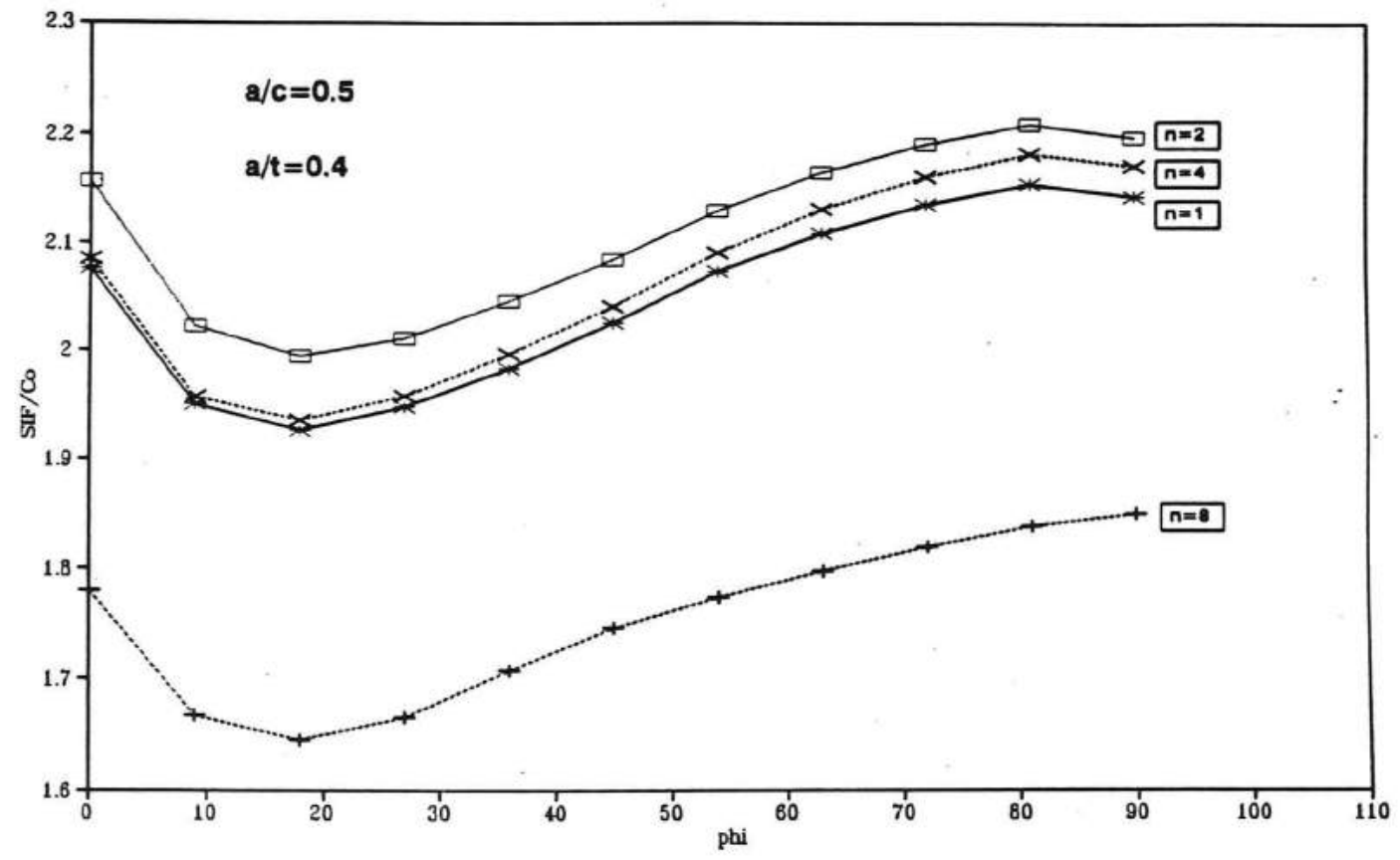

Fig.B.9 Normalized stress intensity factor vs. $\phi(a / c=0.5, a / t=0.4)$

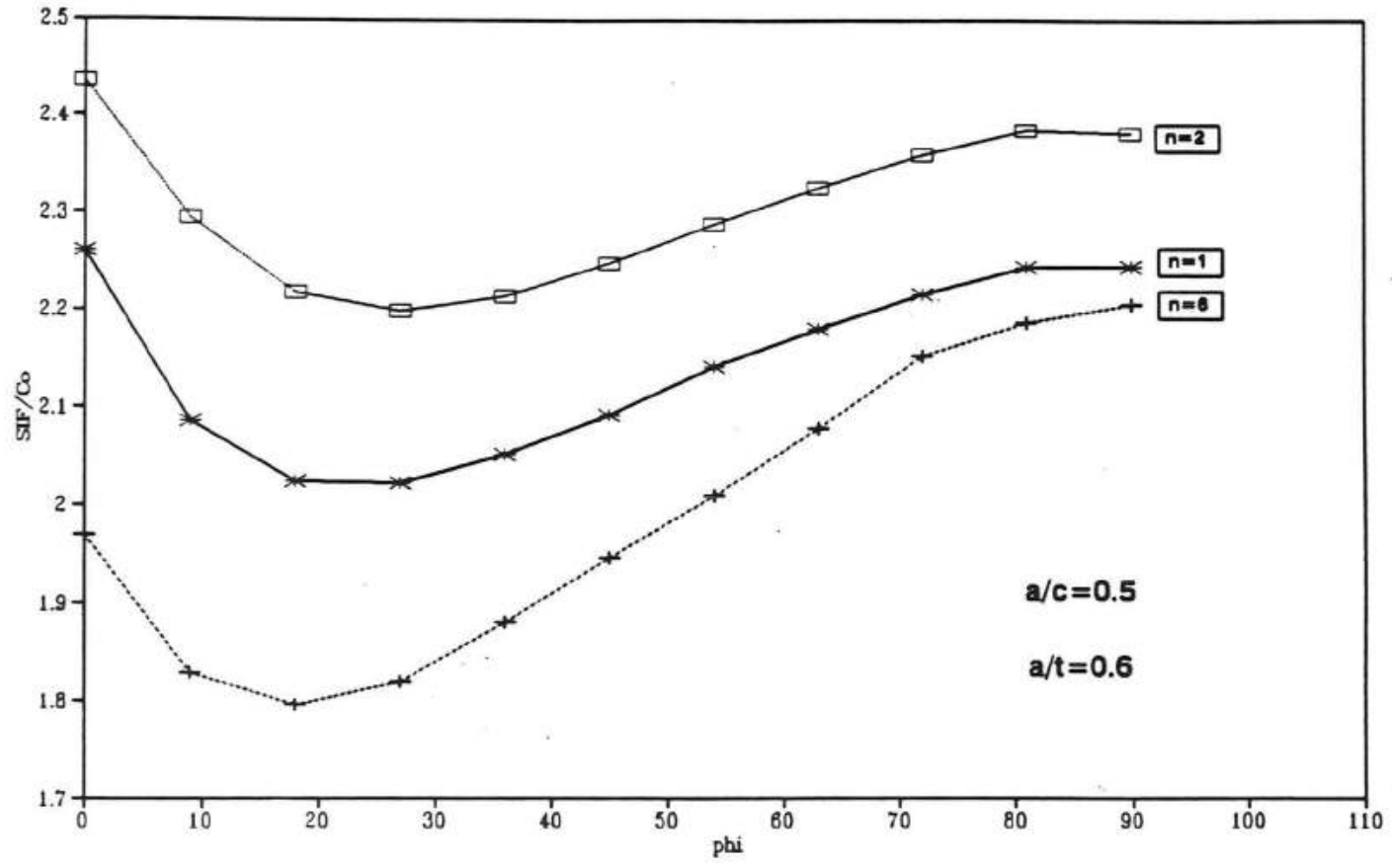

Fig. B. 10 Normalized stress intensity factor vs. $\phi(a / c=0.5, a / t=0.6)$ 


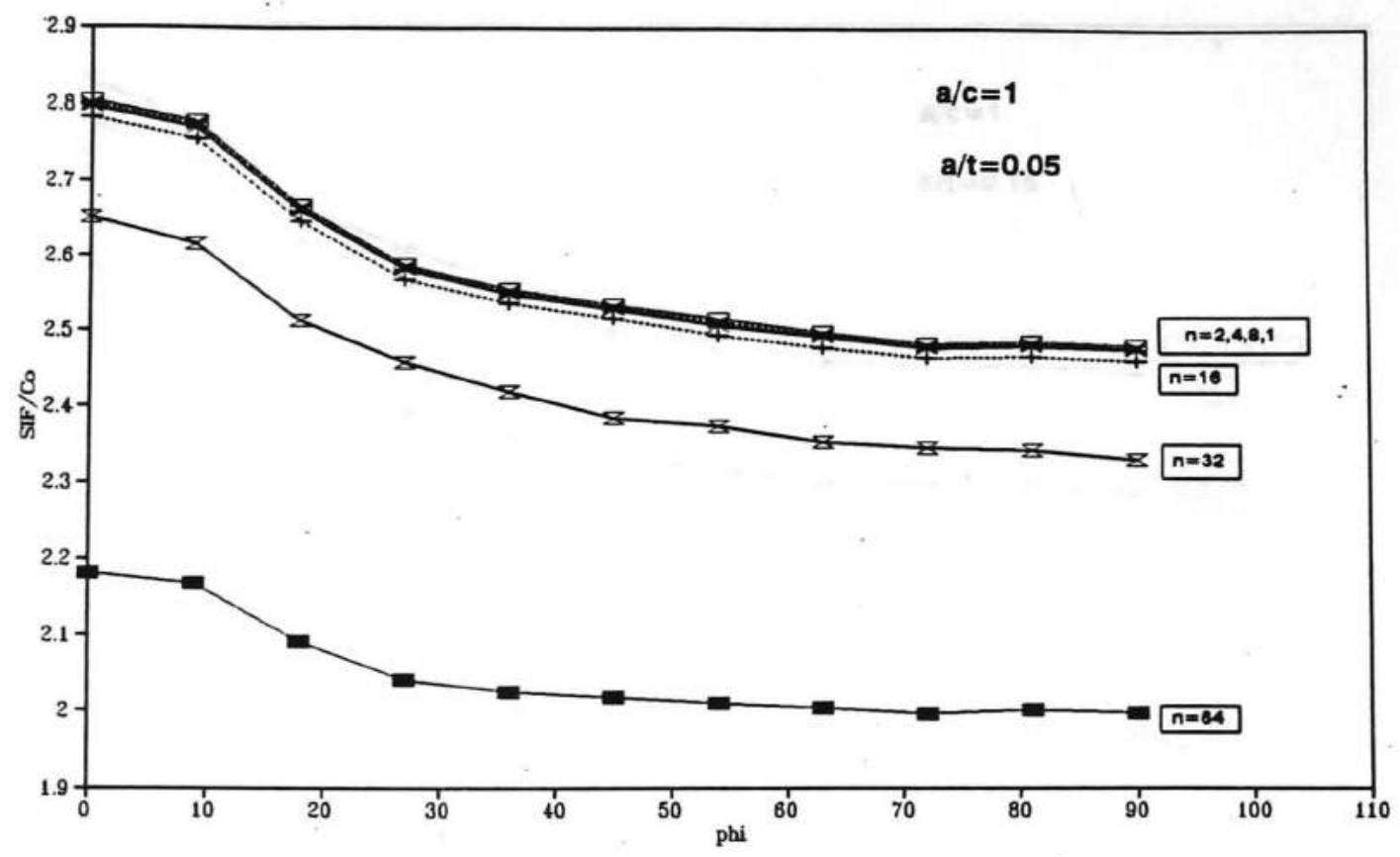

Fig.B.11 Normalized stress intensity factor vs. $\phi(a / c=1, a / t=0.05)$

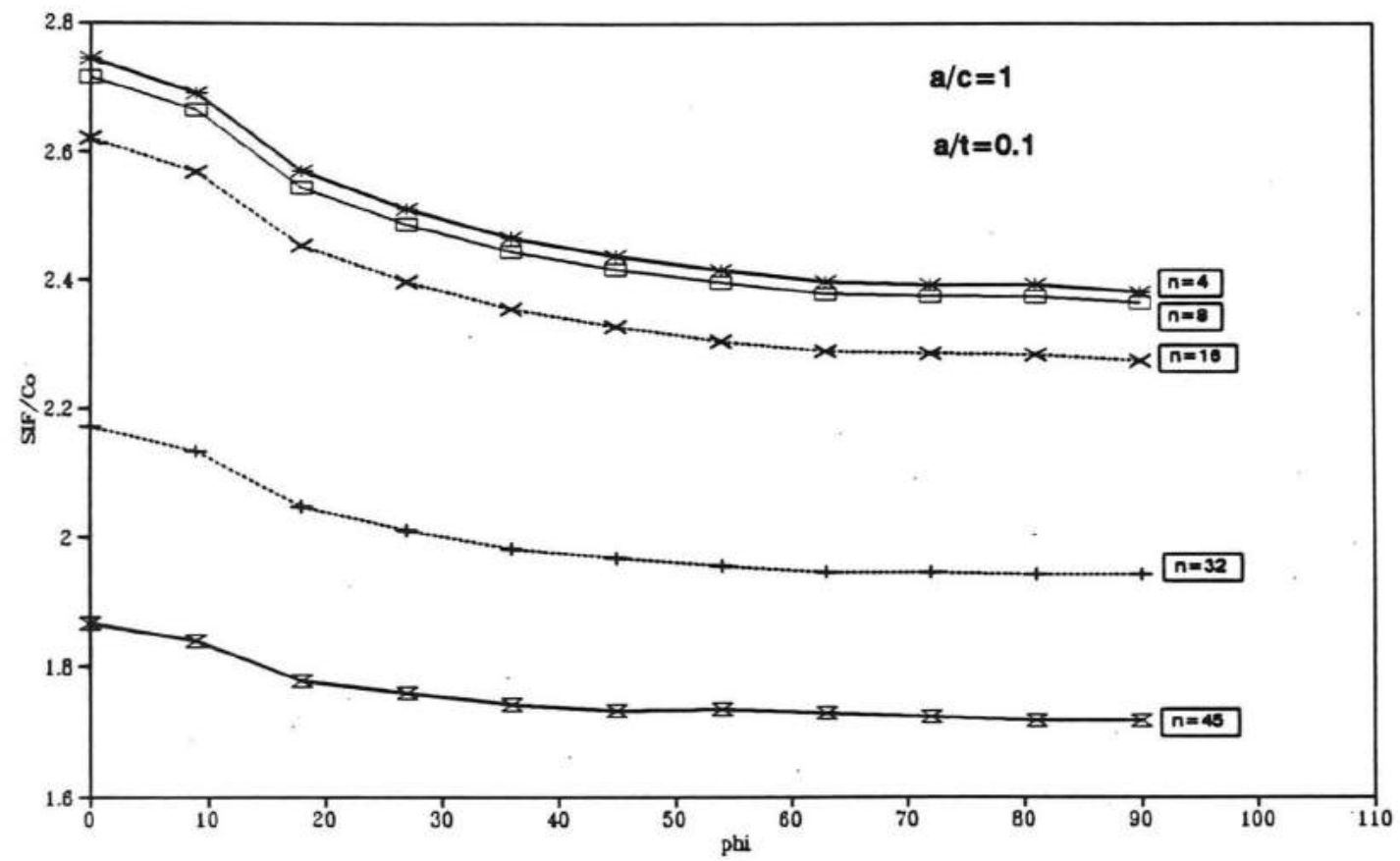

Fig.B.12 Normalized stress intensity factor vs. $\phi(a / c=1, a / t=0.1)$ 


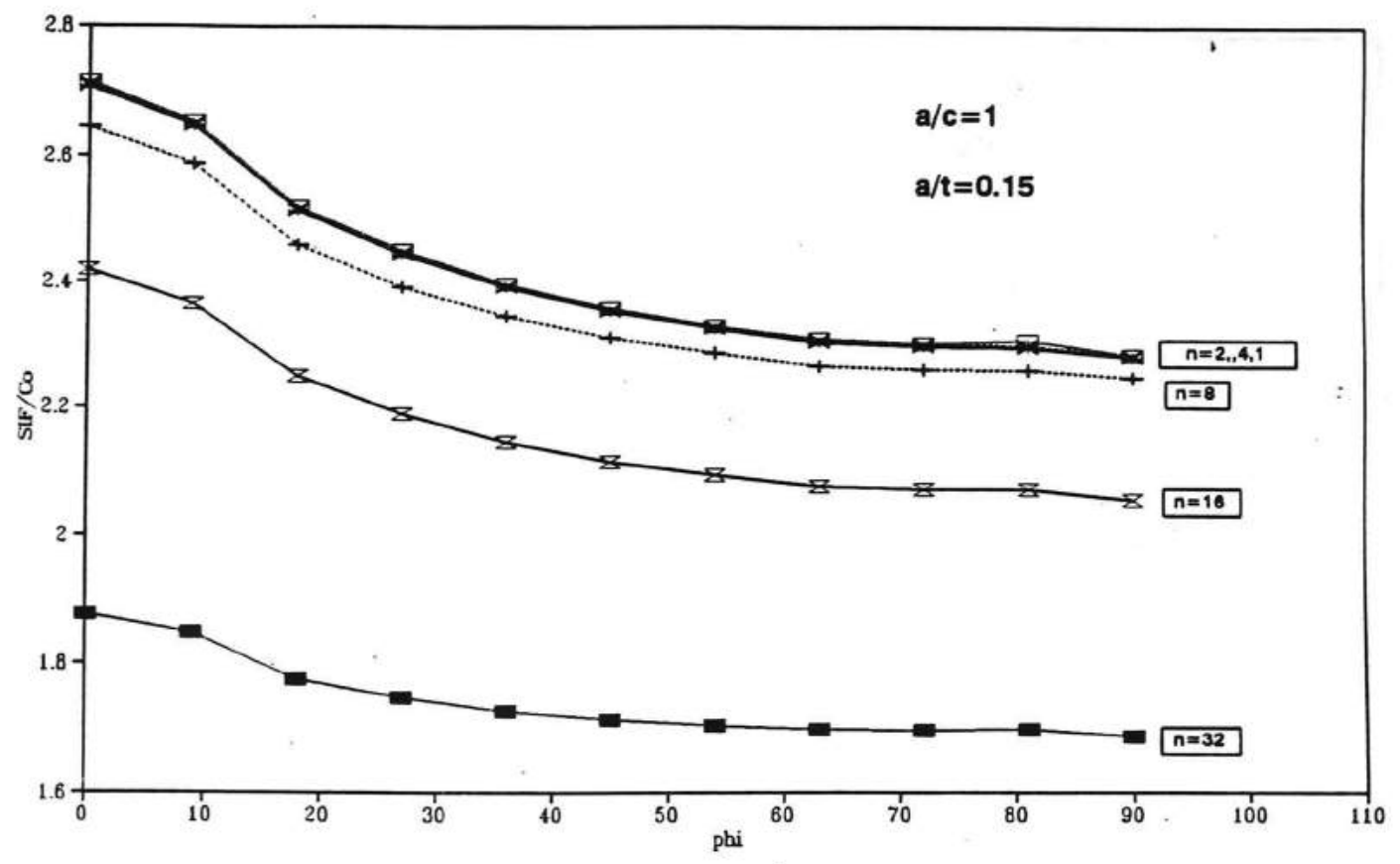

Fig.B.13 Normalized stress intensity factor vs. $\phi(a / c=1, a / t=0.15)$

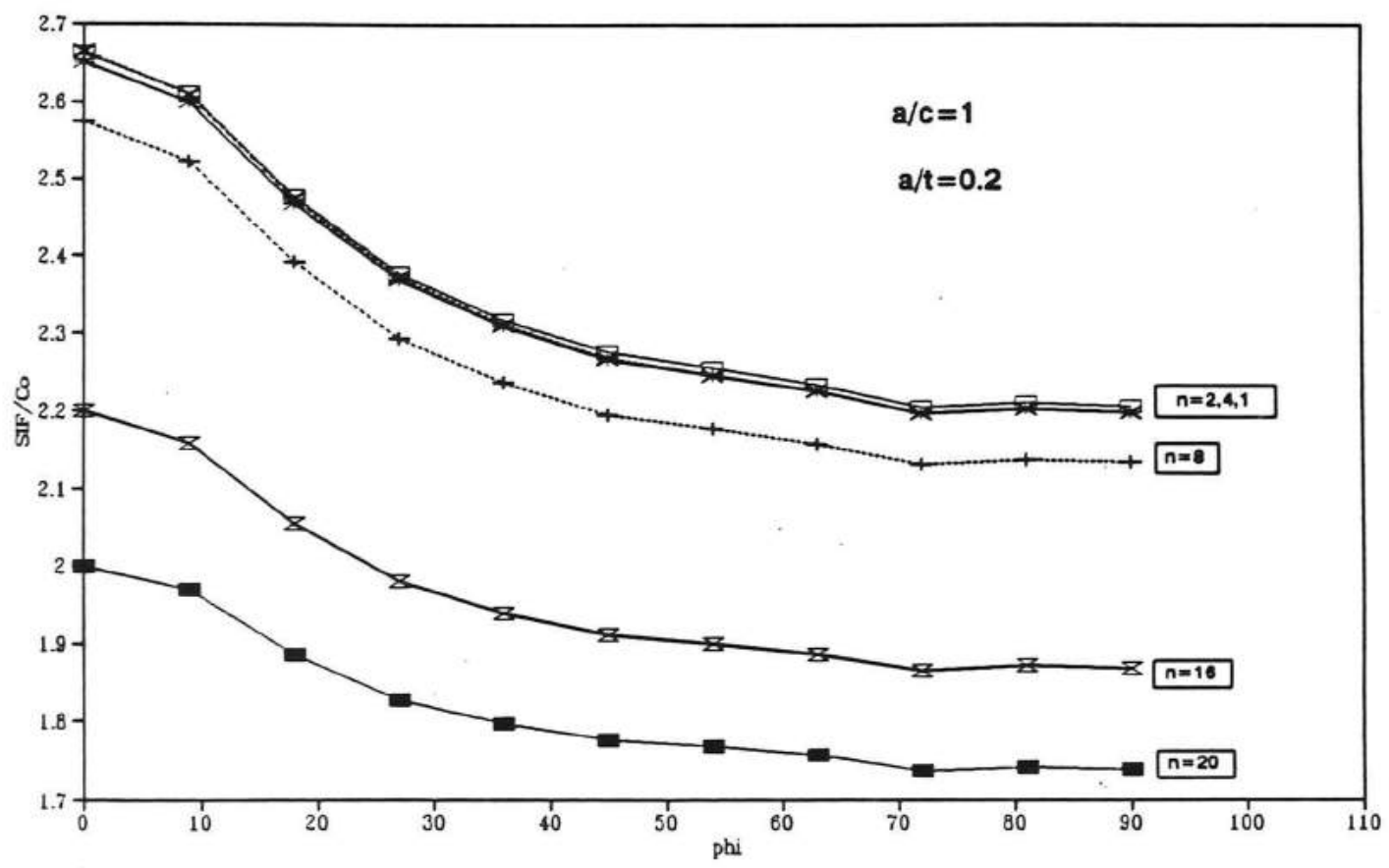

Fig.B.14 Normalized stress intensity factor vs. $\phi(a / c=1, a / t=0.2)$ 


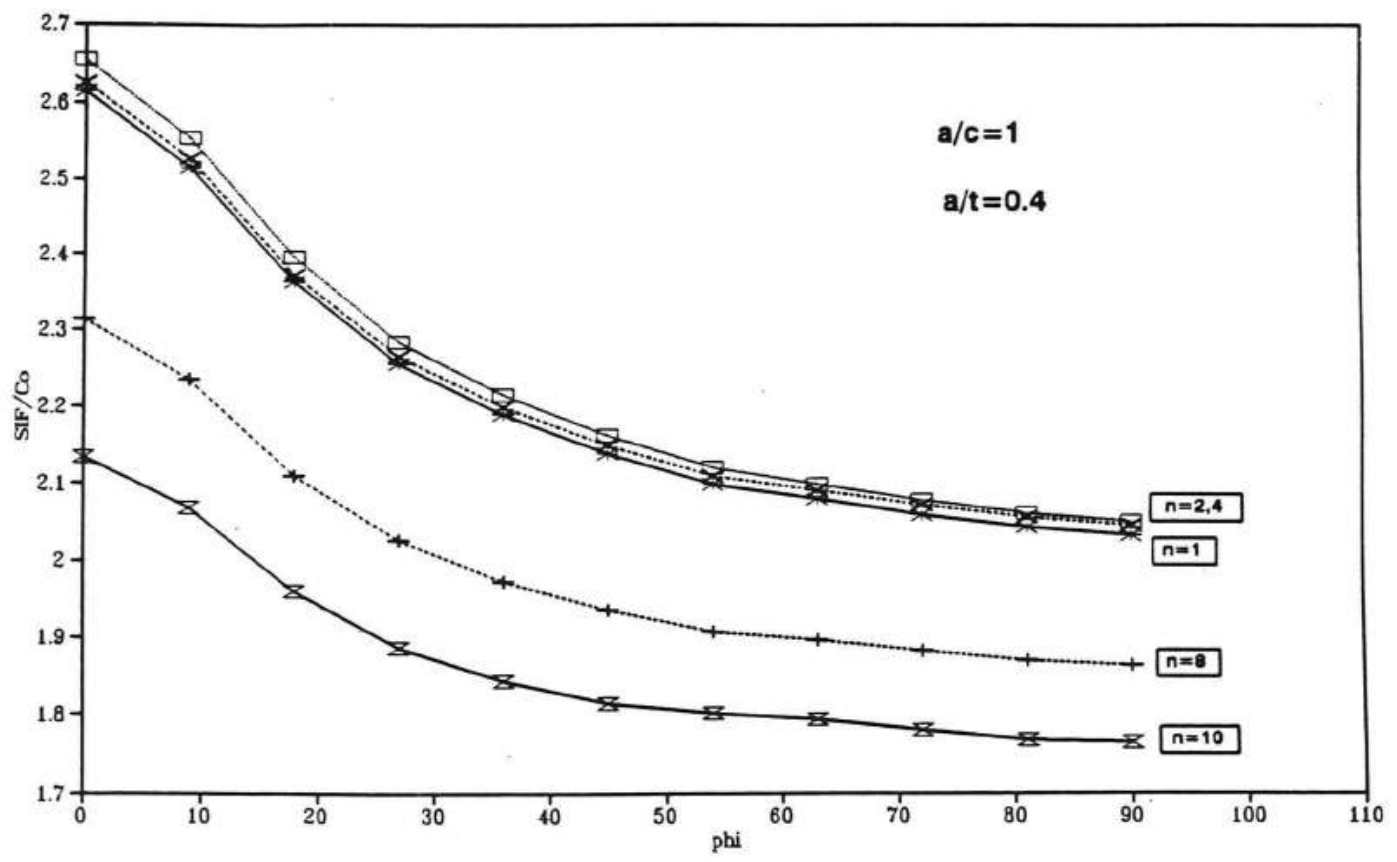

Fig.B.15 Normalized stress intensity factor vs. $\phi(a / c=1, a / t=0.4)$

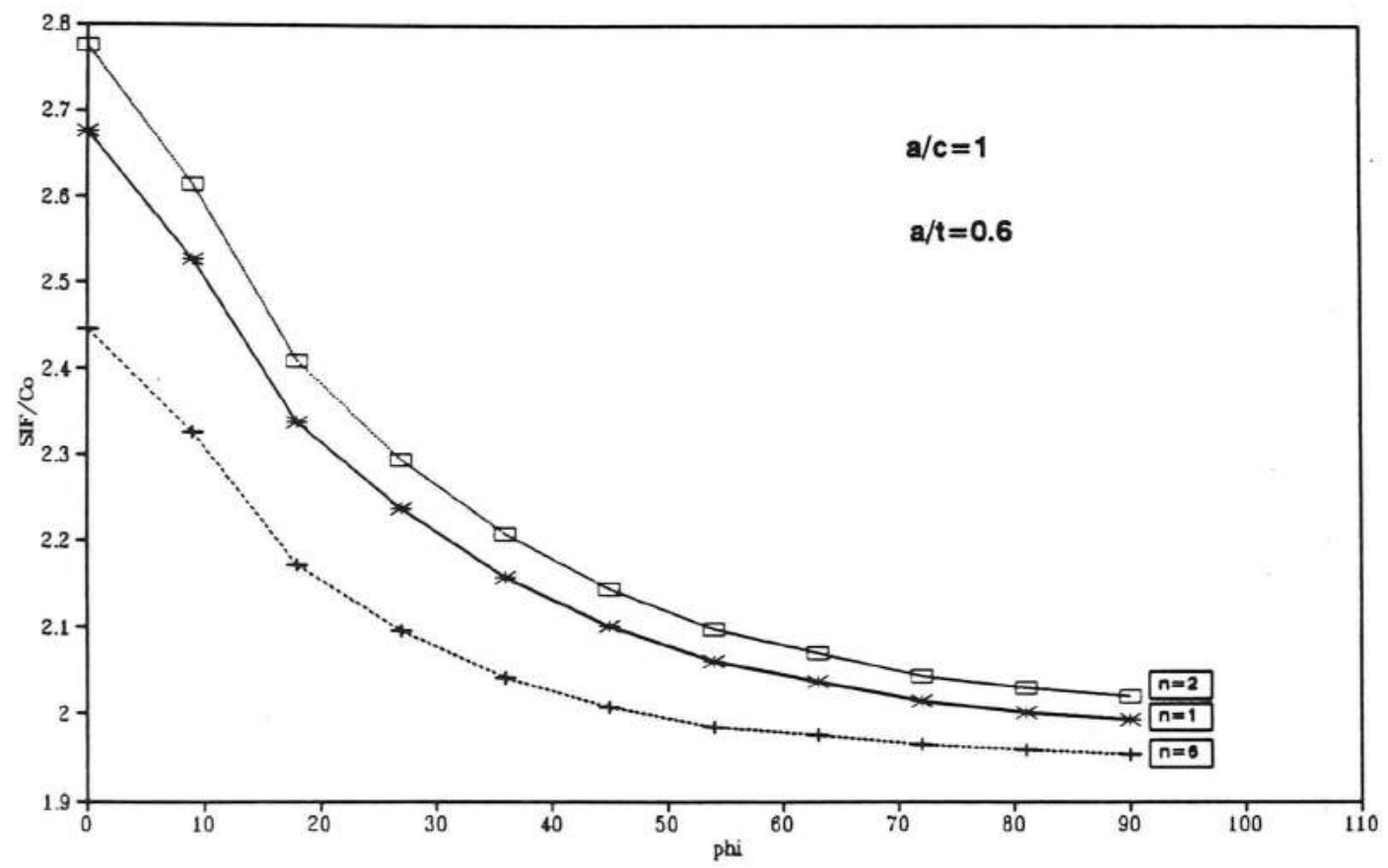

Fig.B.16 Normalized stress intensity factor vs. $\phi(a / c=1, a / t=0.6)$ 


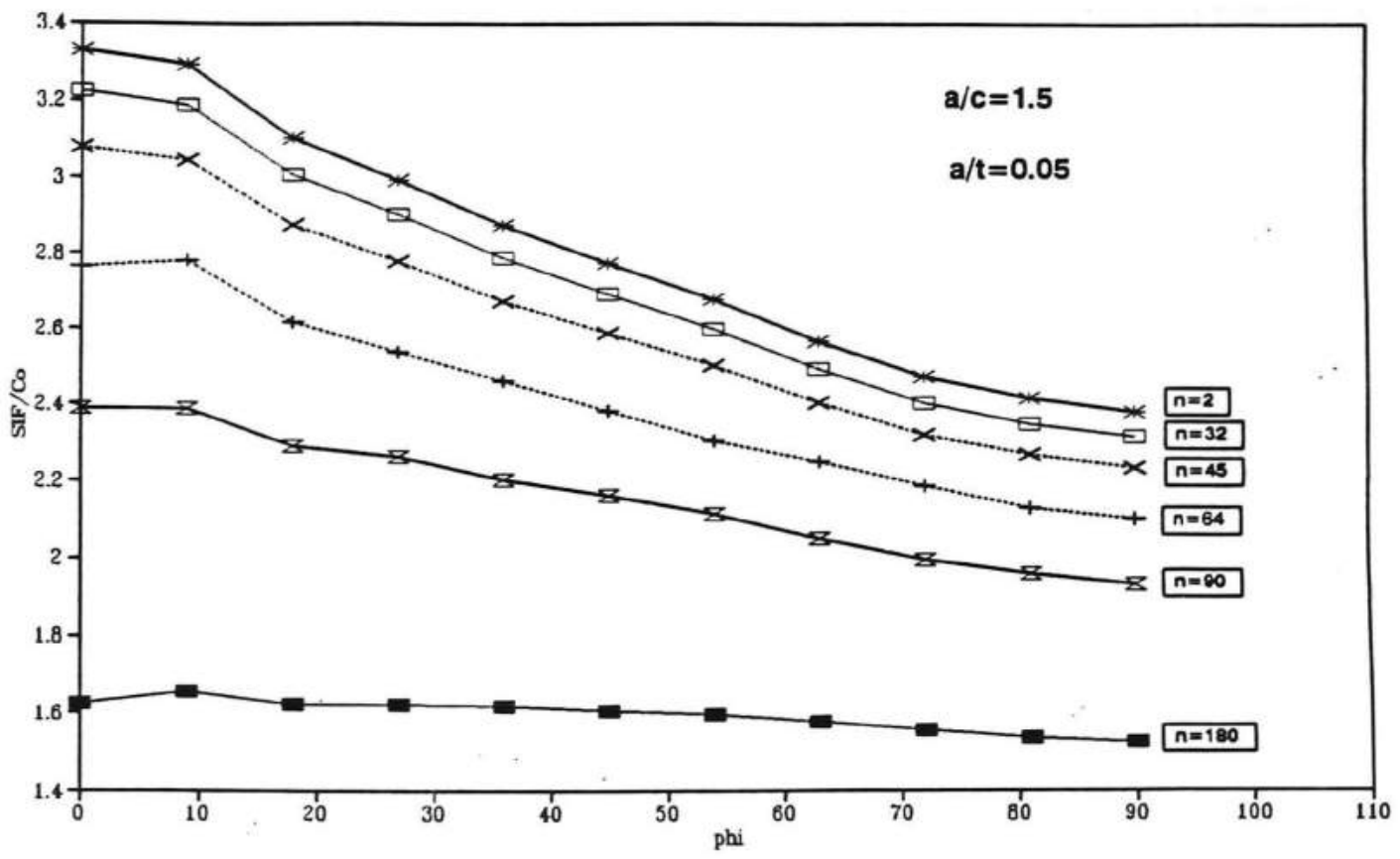

Fig. B. 17 Normalized stress intensity factor $v s . \phi(a / c=1.5, a / t=0.05)$

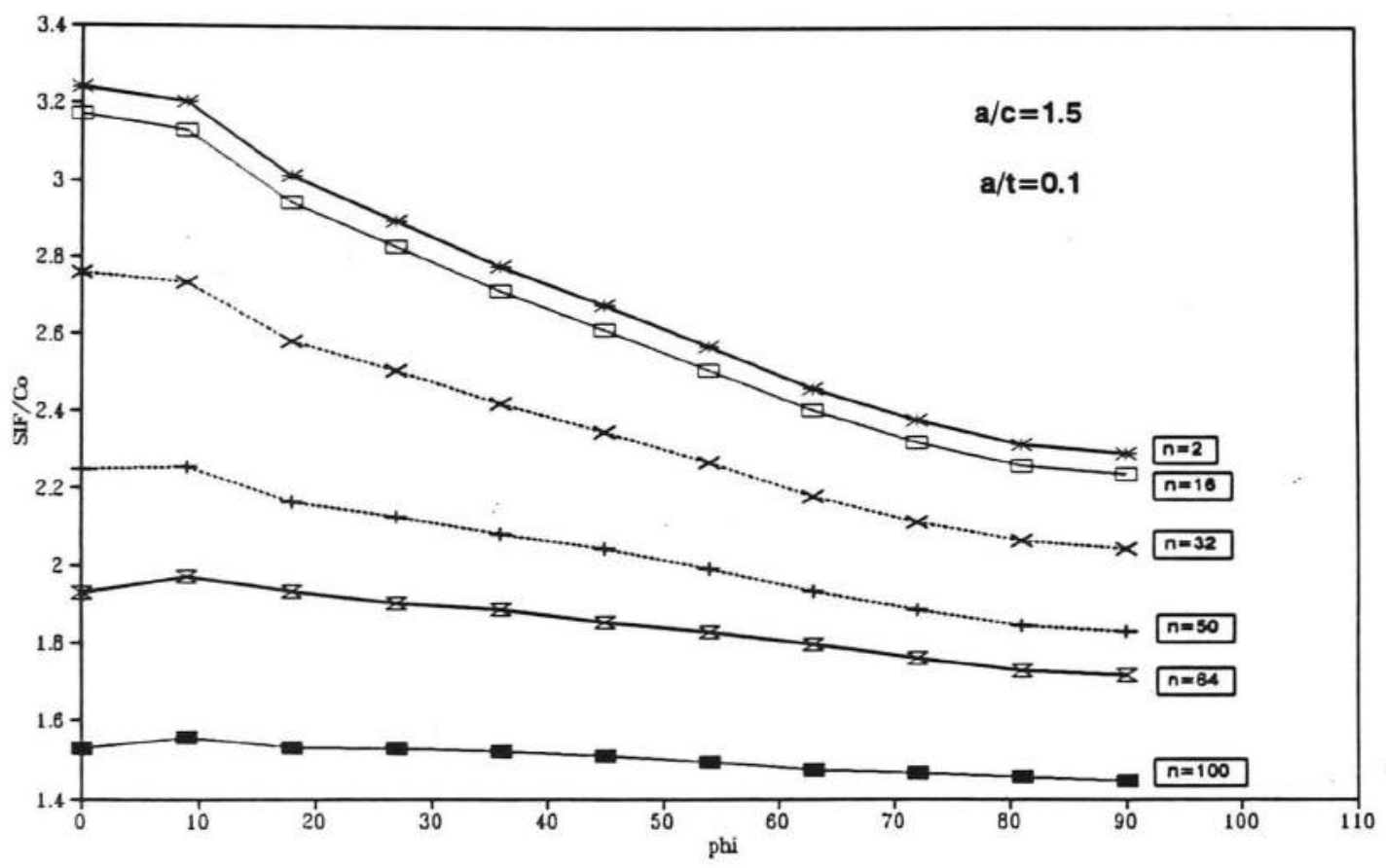

Fig. B. 18 Normalized stress intensity factor vs. $\phi(a / c=1.5, a / t=0.1)$ 


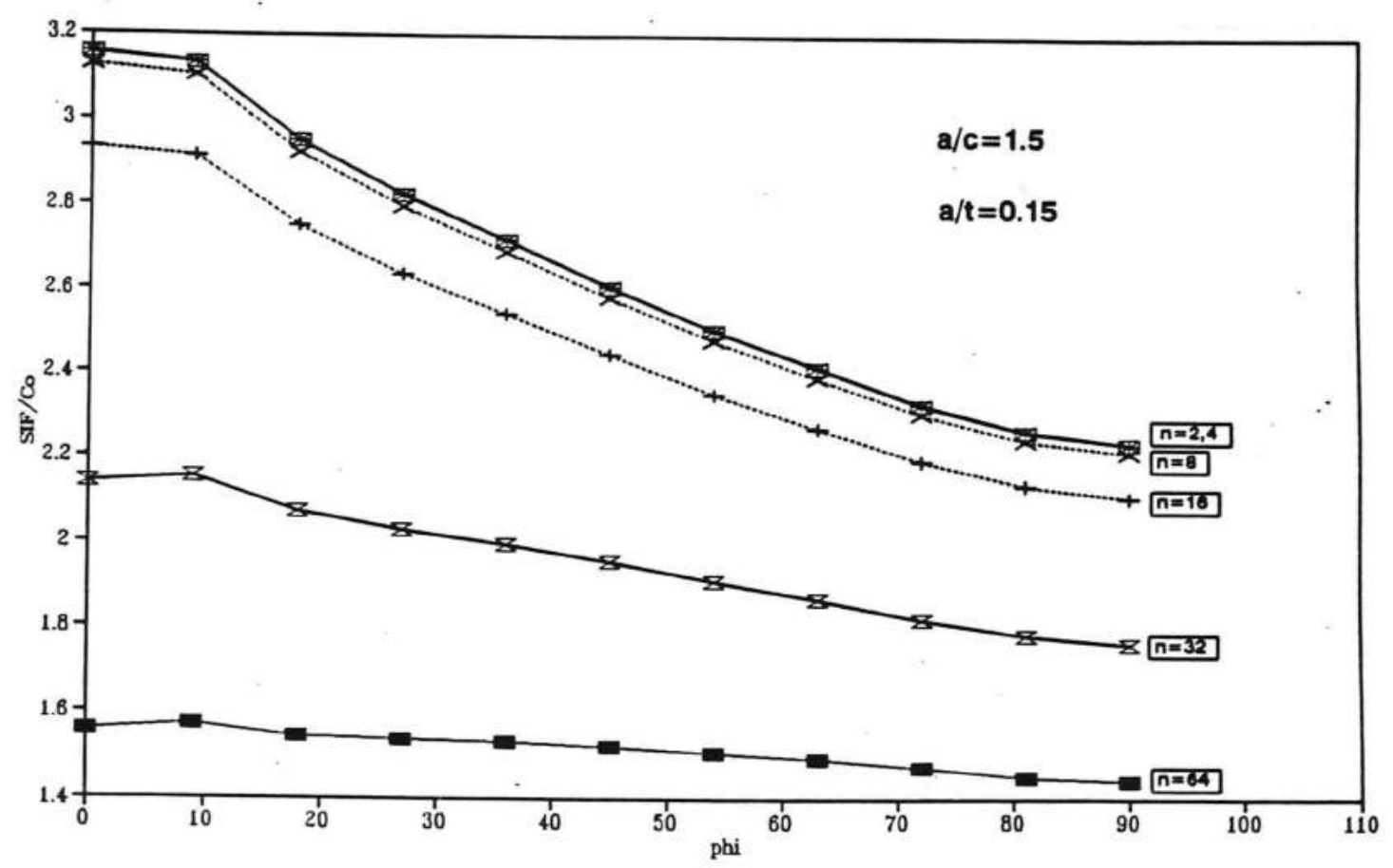

Fig.B.19 Normalized stress intensity factor vs. $\phi(a / c=1.5, a / t=0.15)$

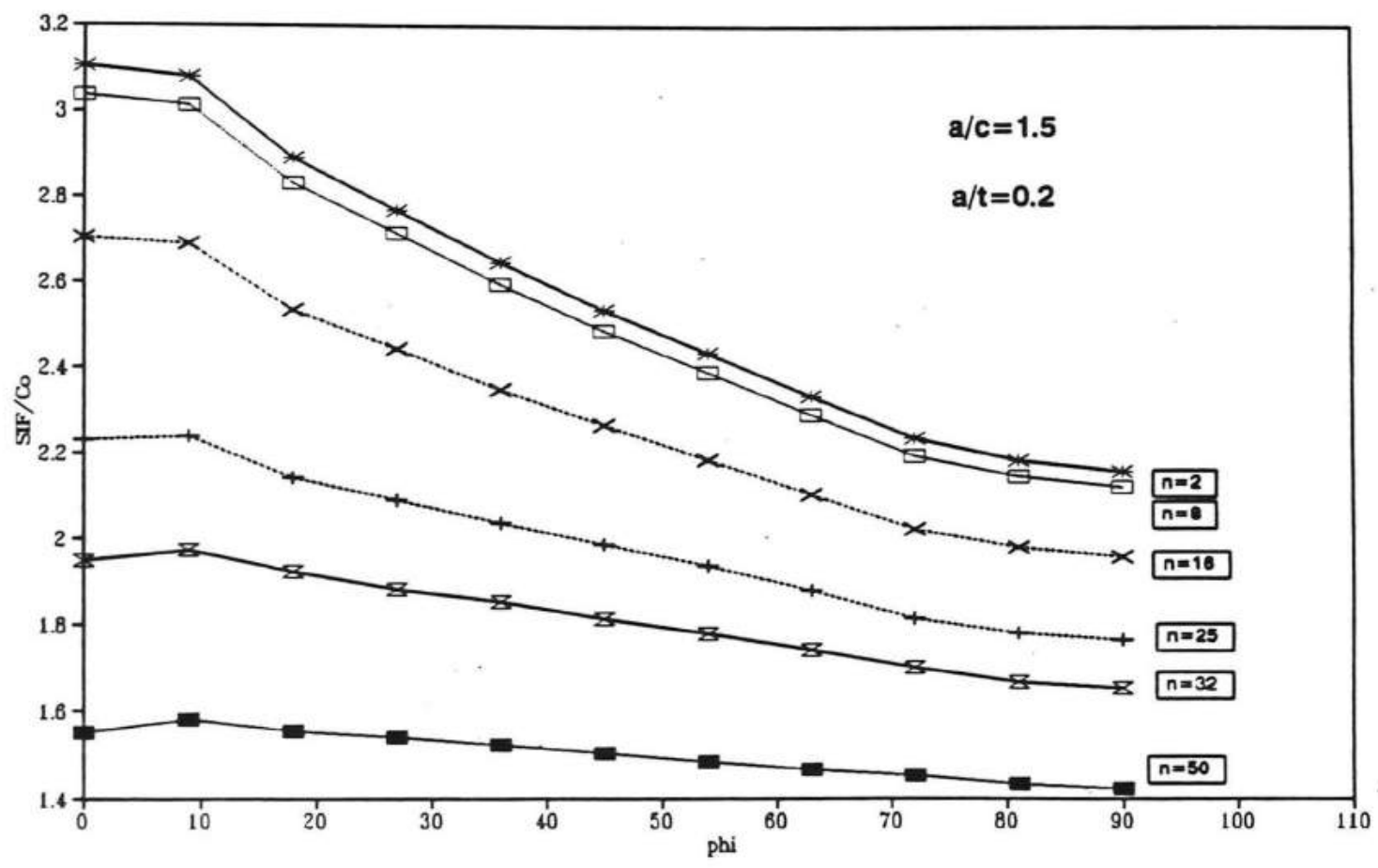

Fig.B.20 Normalized stress intensity factor vs. $\phi(a / c=1.5, a / t=0.2)$ 


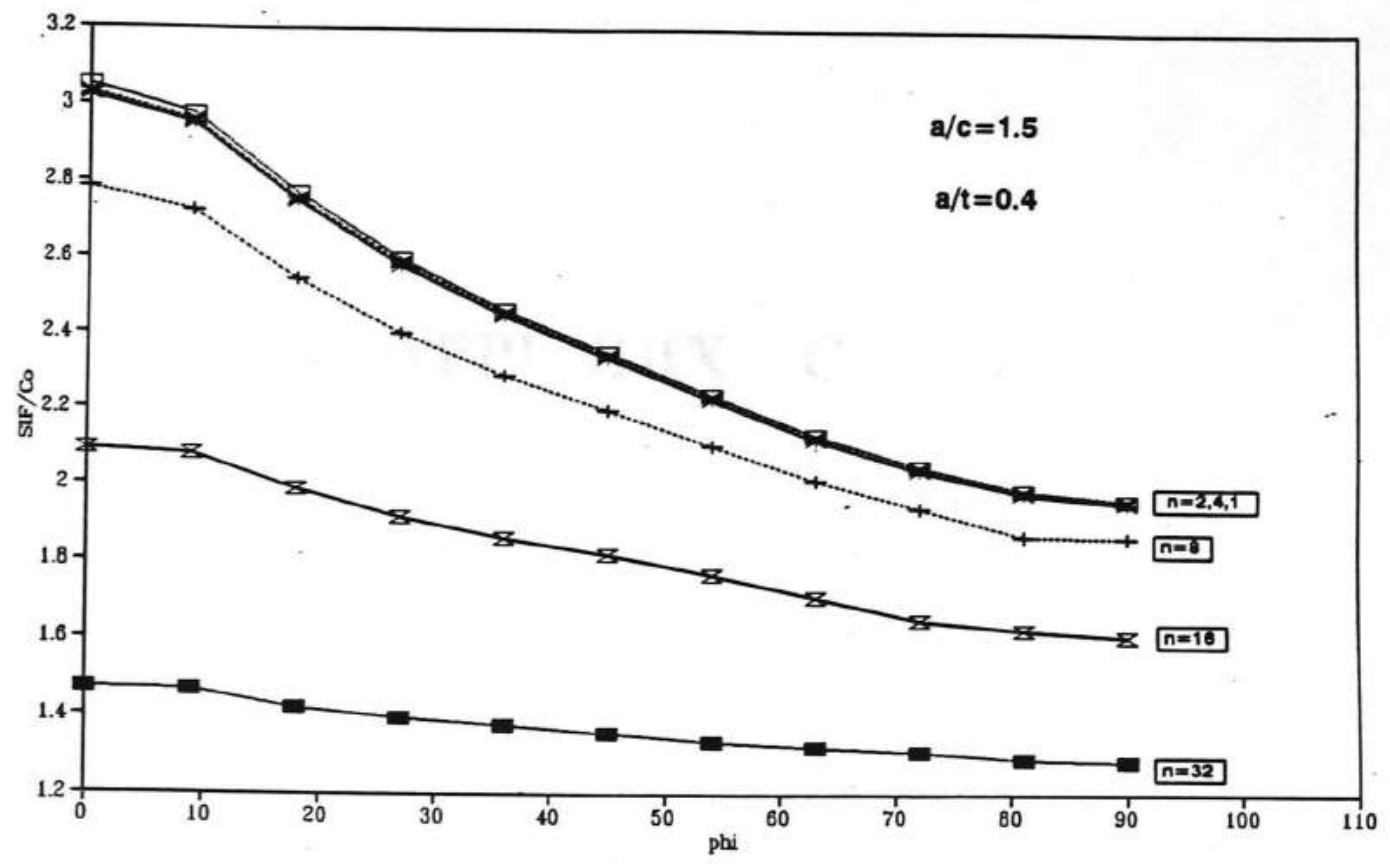

Fig.B.21 Normalized stress intensity factor vs. $\phi(a / c=1.5, a / t=0.4)$

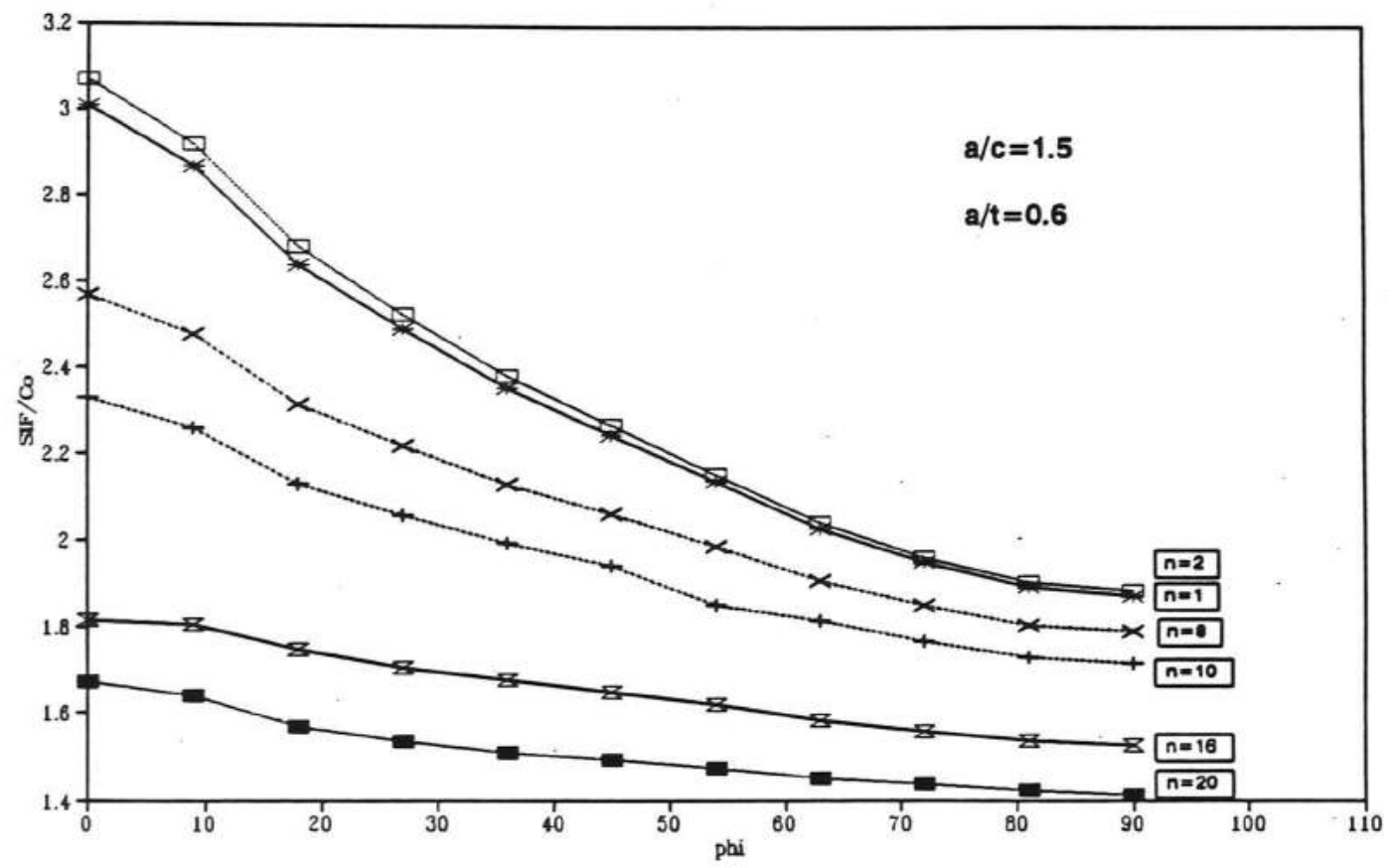

Fig.B.22 Normalized stress intensity factor vs. $\phi(a / c=1.5, a / t=0.6)$ 


\section{APPENDIX C}

MACROS 


\section{MACROS}

These macros are used to create the submodel and are divided as following:

MESH, to create a polar coordinate system every ten degrees.

NMESH0, to create the nodes.

NMESH1, to create the midside nodes.

NMESH2, to create the elements. 


\section{MESH}

$==\mathbf{=}=\mathbf{=}=\mathbf{=}=\mathbf{=}=\mathrm{=}=\mathrm{=}=$

csys, 1

$\mathrm{k}, 1, \mathrm{ri}, 180$

/view, $1,1, .5,1$

csys, 0

local, 14,0,-ri, 0,0,0,-90,-90

*set, q,0

*set, ki, 10

:bra

$\mathrm{k}, \mathrm{ki}, \mathrm{c} * \cos (\mathrm{q} * \mathrm{pi} / 180), \mathrm{a} * \sin (\mathrm{q} * \mathrm{pi} / 180)$

*set, q, q+9

*set, ki, ki+10

*if, q,le, 90 ,:bra

/pnum,kpoi, 1

kscale, $200,10,110,10,5 / 3,5 / 3$

kgen, $2,10,110,10,,, c, 400$

kplot

mp,ex, $1, \mathrm{e}$

mp, nuxy, 1, nu

\section{NMESHO}

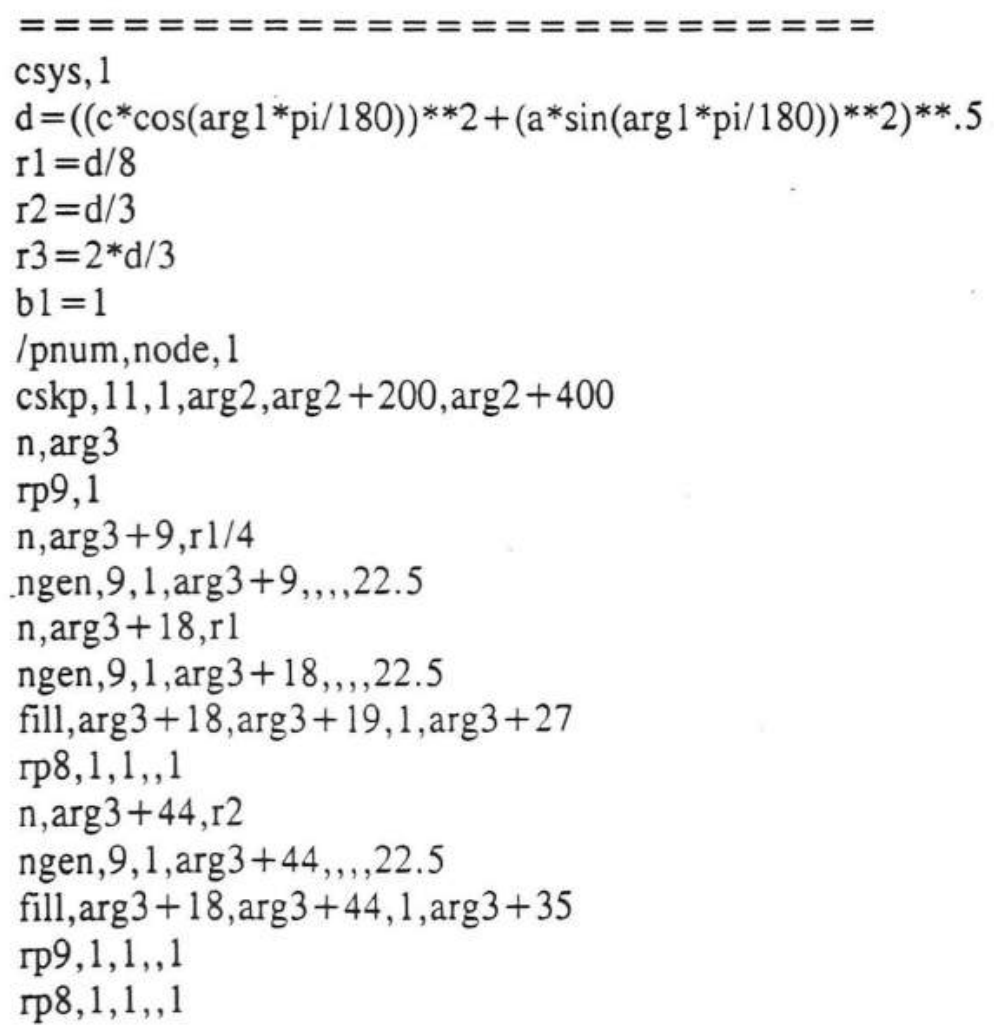


$\mathrm{n}, \arg 3+1254, \mathrm{r} 3$

ngen, $9,1, \arg 3+1254,,,, 22.5$

fill, $\arg 3+44, \arg 3+1254,1, \arg 3+61$

rp $9,1,1,, 1$

fill, arg $3+1254, \arg 3+1255,1, \arg 3+1263$

гp $8,1,1,1$

\section{NMESH1}

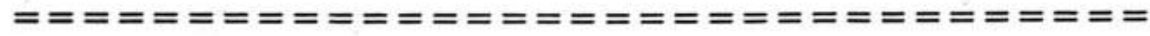

fill, $\arg 1, \arg 1+100,1, \arg 1+70$

rp $9,1,1,1$

fill, $\arg 1+18, \arg 1+118,1, \arg 1+79$

rp $9,1,1,1$

fill, arg $1+44, \arg 1+144,1, \arg 1+88$

гр9, $1,1,1$

fill, arg $1+1254, \arg 1+1354,1, \arg 1+1280$

rp $9,1,1,, 1$

/pnum, node, 0

\section{NMESH2}

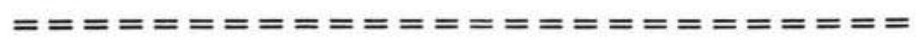

nplot

et, 1,95

e, $119,120,101,101,19,20,1,1$

emore, $128,111,101,110,28,11,1,10$

emore, $80,81,71,71$

egen, $8,1,-1$

e, $145,146,120,119,45,46,20,19$

emore, $154,137,128,136,54,37,28,36$

emore, $89,90,81,80$

egen, $8,1,-1$

e, $1355,1356,146,145,1255,1256,46,45$

emore, $1364,163,154,162,1264,63,54,62$

emore, $1281,1282,90,89$

egen, $8,1,-1$

egen, $10,100,-24$

/type, 1,4

/pnum,elem, 1

eplot

numm, node

csys, 0

nsel, node, 1

nasel, node, $1,19,9$ 


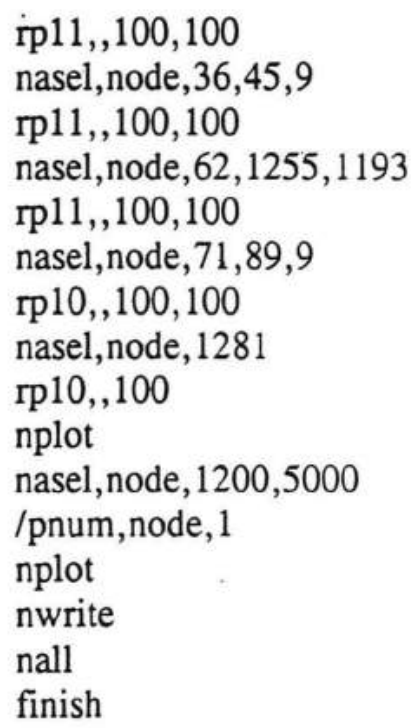




\title{
APPENDIX D
}

FINITE ELEMENT STRESS INTENSITY FACTORS vs.

\author{
EMPIRICAL EQUATION RESULTS
}




\begin{tabular}{|c|c|c|c|c|c|}
\hline$a / c$ & $a / t$ & $\mathrm{n}$ & SIF(F.E.) & SIF(eq.3.26) & Error(\%) \\
\hline \multirow[t]{17}{*}{0.2} & 0.05 & 1 & 2.4632 & 2.4256 & 1.53 \\
\hline & & 2 & 2.4688 & 2.4585 & 0.42 \\
\hline & & 4 & 2.4663 & 2.4949 & -1.16 \\
\hline & & 8 & 2.4527 & 2.4902 & -1.53 \\
\hline & & 16 & 2.3645 & 2.3459 & 0.79 \\
\hline & & 32 & 1.9853 & 1.9854 & -0.01 \\
\hline & 0.1 & 2 & 2.4132 & 2.3767 & 1.51 \\
\hline & & 4 & 2.4045 & 2.4128 & -0.35 \\
\hline & & 8 & 2.3324 & 2.2989 & 1.44 \\
\hline & & 16 & 1.9554 & 1.9736 & -0.93 \\
\hline & 0.15 & 2 & 2.3729 & 2.4128 & -1.68 \\
\hline & & 4 & 2.3568 & 2.3507 & 0.26 \\
\hline & & 6 & 2.2896 & 2.2543 & 1.54 \\
\hline & 0.2 & 1 & 2.316 & 2.3441 & -1.21 \\
\hline & & 2 & 2.3537 & 2.3603 & -0.28 \\
\hline & & 4 & 2.3288 & 2.2793 & 2.13 \\
\hline & & 8 & 1.9918 & 2.006 & -0.71 \\
\hline \multirow[t]{17}{*}{0.5} & 0.05 & 1 & 2.4975 & 2.4346 & 2.52 \\
\hline & & 2 & 2.5035 & 2.4805 & 0.92 \\
\hline & & 4 & 2.5065 & 2.5359 & -1.17 \\
\hline & & 8 & 2.5074 & 2.5547 & -1.89 \\
\hline & & 16 & 2.4689 & 2.439 & 1.21 \\
\hline & & 32 & 2.2253 & 2.1237 & 4.57 \\
\hline & & 45 & 1.9811 & 1.9177 & 3.20 \\
\hline & & 64 & 1.6896 & 1.6997 & -0.60 \\
\hline & & 90 & 1.4527 & 1.5039 & -3.52 \\
\hline & 0.1 & 1 & 2.3201 & 2.3464 & -1.13 \\
\hline & & 2 & 2.3295 & 2.399 & -2.98 \\
\hline & & 4 & 2.3559 & 2.4317 & -3.22 \\
\hline & & 8 & 2.2966 & 2.3605 & -2.78 \\
\hline & & 16 & 2.1143 & 2.1033 & 0.52 \\
\hline & & 20 & 2.0147 & 1.9844 & 1.50 \\
\hline & & 32 & 1.6715 & 1.7096 & -2.28 \\
\hline & & 40 & 1.5152 & 1.5802 & -4.29 \\
\hline
\end{tabular}




\begin{tabular}{|c|c|c|c|c|c|}
\hline$a / c$ & $\mathrm{a} / \mathrm{t}$ & $\mathrm{n}$ & SIF(F.E.) & SIF(eq.3.26) & Error(\%) \\
\hline \multirow[t]{19}{*}{0.5} & 0.15 & 1 & 2.3258 & 2.2708 & 2.36 \\
\hline & & 2 & 2.3374 & 2.3203 & 0.73 \\
\hline & & 4 & 2.3299 & 2.3252 & 0.20 \\
\hline & & 8 & 2.2493 & 2.193 & 2.50 \\
\hline & & 16 & 1.9312 & 1.8811 & 2.59 \\
\hline & & 32 & 1.4332 & 1.4931 & -4.18 \\
\hline & 0.2 & 1 & 2.2591 & 2.2136 & 2.01 \\
\hline & & 2 & 2.2736 & 2.2576 & 0.70 \\
\hline & & 4 & 2.2656 & 2.2392 & 1.17 \\
\hline & & 8 & 2.1142 & 2.0682 & 2.18 \\
\hline & & 16 & 1.6444 & 1.7344 & -5.47 \\
\hline & & 20 & 1.5246 & 1.6114 & -5.69 \\
\hline & 0.4 & 1 & 2.154 & 2.1765 & -1.04 \\
\hline & & 2 & 2.2088 & 2.202 & 0.31 \\
\hline & & 4 & 2.1815 & 2.1259 & 2.55 \\
\hline & & 8 & 1.8512 & 1.8793 & -1.52 \\
\hline & 0.6 & 1 & 2.2612 & 2.3804 & -5.27 \\
\hline & & 2 & 2.4362 & 2.3951 & 1.69 \\
\hline & & 6 & 2.2055 & 2.1235 & 3.72 \\
\hline \multirow[t]{16}{*}{1} & 0.05 & 1 & 2.7961 & 2.762 & 1.22 \\
\hline & & 2 & 2.8035 & 2.7832 & 0.72 \\
\hline & & 4 & 2.801 & 2.8148 & -0.49 \\
\hline & & 8 & 2.7996 & 2.8436 & -1.57 \\
\hline & & 16 & 2.7816 & 2.8136 & -1.15 \\
\hline & & 32 & 2.6503 & 2.612 & 1.45 \\
\hline & & 64 & 2.1808 & 2.1669 & 0.64 \\
\hline & 0.1 & 1 & 2.7374 & 2.7133 & 0.88 \\
\hline & & 2 & 2.7424 & 2.7432 & -0.03 \\
\hline & & 4 & 2.7433 & 2.7733 & -1.09 \\
\hline & & 8 & 2.7158 & 2.7555 & -1.46 \\
\hline & & 16 & 2.6211 & 2.5842 & 1.41 \\
\hline & & 32 & 2.1707 & 2.1752 & -0.21 \\
\hline & & 45 & 1.8679 & 1.909 & -2.20 \\
\hline & 0.15 & 1 & 2.7066 & 2.6737 & 1.22 \\
\hline & & 2 & 2.7135 & 2.7059 & 0.28 \\
\hline
\end{tabular}




\begin{tabular}{|c|c|c|c|c|c|}
\hline $\mathrm{a} / \mathrm{c}$ & $\mathrm{a} / \mathrm{t}$ & $\mathrm{n}$ & SIF(F.E.) & SIF(eq.3.26) & Error(\%) \\
\hline \multirow[t]{19}{*}{1} & 0.15 & 4 & 2.7091 & 2.7224 & -0.49 \\
\hline & & 8 & 2.6448 & 2.6483 & -0.13 \\
\hline & & 16 & 2.4193 & 2.3711 & 1.99 \\
\hline & 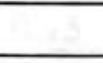 & 32 & 1.8771 & 1.8798 & -0.14 \\
\hline & 0.2 & 1 & 2.6505 & 2.6442 & 0.24 \\
\hline & & 2 & 2.6621 & 2.6753 & -0.50 \\
\hline & & 4 & 2.6636 & 2.6744 & -0.41 \\
\hline & & 8 & 2.5738 & 2.5494 & 0.95 \\
\hline & & 16 & 2.2006 & 2.2012 & -0.03 \\
\hline & & 20 & 2.0008 & 2.044 & -2.16 \\
\hline & 0.4 & 1 & 2.6134 & 2.6288 & -0.59 \\
\hline & & 2 & 2.6547 & 2.6459 & 0.33 \\
\hline & & 4 & 2.6248 & 2.5776 & 1.80 \\
\hline & & 8 & 2.3135 & 2.3144 & -0.04 \\
\hline & & 10 & 2.1342 & 2.1798 & -2.14 \\
\hline & 0.6 & 1 & 2.6747 & 2.7557 & -3.03 \\
\hline & & 2 & 2.7751 & 2.757 & 0.65 \\
\hline & & 4 & 2.7128 & 2.6343 & 2.89 \\
\hline & & 6 & 2.446 & 2.459 & -0.53 \\
\hline \multirow[t]{16}{*}{1.5} & 0.05 & 1 & 3.3147 & 3.2581 & 1.71 \\
\hline & & 2 & 3.3282 & 3.2956 & 0.98 \\
\hline & & 4 & 3.3261 & 3.354 & -0.84 \\
\hline & & 8 & 3.3255 & 3.4189 & -2.81 \\
\hline & & 16 & 3.3144 & 3.419 & -3.16 \\
\hline & & 32 & 3.2239 & 3.2115 & 0.38 \\
\hline & & 45 & 3.0772 & 2.9978 & 2.58 \\
\hline & & 64 & 2.7815 & 2.7079 & 2.65 \\
\hline & & 90 & 2.3871 & 2.387 & 0.00 \\
\hline & & 180 & 1.6588 & 1.7464 & -5.28 \\
\hline & 0.1 & 1 & 3.2353 & 3.1664 & 2.13 \\
\hline & & 2 & 3.2413 & 3.2211 & 0.62 \\
\hline & & 4 & 3.2358 & 3.2845 & -1.51 \\
\hline & & 8 & 3.2367 & 3.2396 & -0.09 \\
\hline & & 16 & 3.1688 & 3.1142 & 1.72 \\
\hline & & 32 & 2.7573 & 2.6498 & 3.90 \\
\hline
\end{tabular}




\begin{tabular}{|c|c|c|c|c|c|}
\hline$a / c$ & $\mathrm{a} / \mathrm{t}$ & $\mathrm{n}$ & SIF(F.E.) & SIF(eq.3.26) & Error(\%) \\
\hline \multirow[t]{31}{*}{1.5} & 0.1 & 50 & 2.2535 & 2.247 & 0.29 \\
\hline & & 64 & 1.973 & 2.0189 & -2.33 \\
\hline & & 100 & 1.5578 & 1.6382 & -5.16 \\
\hline & 0.15 & 1 & 3.1562 & 3.1001 & 1.78 \\
\hline & & 2 & 3.156 & 3.1611 & -0.16 \\
\hline & & 4 & 3.1537 & 3.2081 & -1.72 \\
\hline & & 8 & 3.1234 & 3.1417 & -0.59 \\
\hline & & 16 & 2.9347 & 2.8265 & 3.69 \\
\hline & & 32 & 2.1552 & 2.2646 & -5.08 \\
\hline & & 64 & 1.5733 & 1.6617 & -5.62 \\
\hline & 0.2 & 1 & 3.087 & 3.0574 & 0.96 \\
\hline & & 2 & 3.1036 & 3.1186 & -0.48 \\
\hline & & 4 & 3.0935 & 3.1411 & -1.54 \\
\hline & & 8 & 3.0371 & 3.0043 & 1.08 \\
\hline & & 16 & 2.7017 & 2.5993 & 3.79 \\
\hline & & 25 & 2.2409 & 2.2254 & 0.69 \\
\hline & & 32 & 1.9748 & 2.0072 & -1.64 \\
\hline & & 50 & 1.5807 & 1.6338 & -3.36 \\
\hline & 0.4 & 1 & 3.0216 & 3.049 & -0.91 \\
\hline & & 2 & 3.0498 & 3.0889 & -1.28 \\
\hline & & 4 & 3.0309 & 3.0066 & 0.80 \\
\hline & & 8 & 2.7818 & 2.6758 & 3.81 \\
\hline & & 16 & 2.0935 & 2.1203 & -1.28 \\
\hline & & 32 & 1.4704 & 1.5481 & -5.28 \\
\hline & 0.6 & 1 & 3.0077 & 3.0814 & -2.45 \\
\hline &. & 2 & 3.0695 & 3.0915 & -0.72 \\
\hline & & 4 & 3.0352 & 2.9266 & 3.58 \\
\hline & & 8 & 2.569 & 2.4945 & 2.90 \\
\hline & & 10 & 2.3297 & 2.3105 & 0.82 \\
\hline & & 16 & 1.817 & 1.9031 & -4.74 \\
\hline & & 20 & 1.6716 & 1.7172 & -2.73 \\
\hline
\end{tabular}

